\title{
THE BRUNN-MINKOWSKI INEQUALITY AND A MINKOWSKI PROBLEM FOR $\mathcal{A}$-HARMONIC GREEN'S FUNCTION
}

\author{
MURAT AKMAN, JOHN LEWIS, OLLI SAARI, AND ANDREW VOGEL
}

ABSTRACT. In this article we study two classical problems in convex geometry associated to $\mathcal{A}$-harmonic PDEs, quasi-linear elliptic PDEs whose structure is modeled on the $p$-Laplace equation. Let $p$ be fixed with $2 \leq n \leq p<\infty$. For a convex compact set $E$ in $\mathbb{R}^{n}$, we define and then prove the existence and uniqueness of the so called $\mathcal{A}$-harmonic Green's function for the complement of $E$ with pole at infinity. We then define a quantity $\mathrm{C}_{\mathcal{A}}(E)$ which can be seen as the behavior of this function near infinity.

In the first part of this article, we prove that $\mathrm{C}_{\mathcal{A}}(\cdot)$ satisfies the following BrunnMinkowski type inequality

$$
\left[\mathrm{C}_{\mathcal{A}}\left(\lambda E_{1}+(1-\lambda) E_{2}\right)\right]^{\frac{1}{p-n}} \geq \lambda\left[\mathrm{C}_{\mathcal{A}}\left(E_{1}\right)\right]^{\frac{1}{p-n}}+(1-\lambda)\left[\mathrm{C}_{\mathcal{A}}\left(E_{2}\right)\right]^{\frac{1}{p-n}}
$$

when $n<p<\infty, 0 \leq \lambda \leq 1$, and $E_{1}, E_{2}$ are nonempty convex compact sets in $\mathbb{R}^{n}$. While $p=n$ then

$$
\mathrm{C}_{\mathcal{A}}\left(\lambda E_{1}+(1-\lambda) E_{2}\right) \geq \lambda \mathrm{C}_{\mathcal{A}}\left(E_{1}\right)+(1-\lambda) \mathrm{C}_{\mathcal{A}}\left(E_{2}\right)
$$

where $0 \leq \lambda \leq 1$ and $E_{1}, E_{2}$ are convex compact sets in $\mathbb{R}^{n}$ containing at least two points.

Moreover, if equality holds in the either of the above inequalities for some $E_{1}$ and $E_{2}$, then under certain regularity and structural assumptions on $\mathcal{A}$ we show that these two sets are homothetic.

The classical Minkowski problem asks for necessary and sufficient conditions on a non-negative Borel measure on the unit sphere $\mathbb{S}^{n-1}$ to be the surface area measure of a convex compact set in $\mathbb{R}^{n}$ under the Gauss mapping for the boundary of this convex set. In the second part of this article we study a Minkowski type problem for a measure associated to the $\mathcal{A}$-harmonic Green's function for the complement of a convex compact set $E$ when $n \leq p<\infty$. If $\mu_{E}$ denotes this measure, then we show that necessary and sufficient conditions for existence under this setting are exactly the same conditions as in the classical Minkowski problem. Using the Brunn-Minkowski inequality result from the first part, we also show that this problem has a unique solution up to translation.

2010 Mathematics Subject Classification. 35J60,31B15,39B62,52A40,35J20,52A20,35J92.

Key words and phrases. The Brunn-Minkowski inequality, Minkowski Problem, Inequalities and extremum problems, Potentials and capacities, $\mathcal{A}$-harmonic PDEs, $\mathcal{A}$-harmonic Green's function, Variational formula, Hadamard variational formula. 


\section{Contents}

\section{Part 1. The Brunn-Minkowski inequality for $\mathcal{A}$-harmonic Green's} function

1. Introduction

2. Notation and statement of results

3. Basic estimates for $\mathcal{A}$-harmonic functions

4. Existence and uniqueness of a fundamental solution 10

4.1. Existence and uniqueness for $p=n \quad 10$

4.2. Existence and uniqueness for $p>n \quad 23$

5. $\mathcal{A}$-harmonic Green's function 25

6. Proof of Theorem A 31

6.1. Proof of the Brunn-Minkowski inequality 34

6.2. Final proof of Theorem A 36

6.2.1. Construction of the fundamental solution 36

Part 2. A Minkowski problem for $\mathcal{A}$-harmonic Green's function 41

7. Introduction and statement of results 41

8. Boundary behavior of $\mathcal{A}$-harmonic functions in Lipschitz domains 43

9. Weak convergence of certain measures on $\mathbb{S}^{n-1} \quad 48$

10. The Hadamard variational formula for $\mathcal{A}$-harmonic PDEs 49

11. Proof of Theorem B 57

11.1. Proof of existence in Theorem B in the discrete case 58

11.2. Existence in Theorem B in the continuous case 65

11.3. Uniqueness in Theorem B 73

$\begin{array}{ll}\text { Acknowledgment } & 73\end{array}$

$\begin{array}{ll}\text { References } & 74\end{array}$

\section{Part 1. The Brunn-Minkowski inequality for $\mathcal{A}$-harmonic Green's} function

\section{INTRODUCTION}

The classical Brunn-Minkowski inequality states that

$$
\left[\operatorname{Vol}\left(\lambda E_{1}+(1-\lambda) E_{2}\right)\right]^{\frac{1}{n}} \geq \lambda\left[\operatorname{Vol}\left(E_{1}\right)\right]^{\frac{1}{n}}+(1-\lambda)\left[\operatorname{Vol}\left(E_{2}\right)\right]^{\frac{1}{n}}
$$

whenever $E_{1}, E_{2}$ are compact convex sets with nonempty interiors in $\mathbb{R}^{n}$ and $\lambda \in[0,1]$. Here $\operatorname{Vol}(\cdot)$ denotes the usual volume in $\mathbb{R}^{n}$ and the summation $\left(\lambda E_{1}+(1-\lambda) E_{2}\right)$ should be understood as a vector sum(called Minkowski addition). Moreover, equality in (1.1) holds precisely when $E_{1}$ and $E_{2}$ are equal up to translation and dilatation (i.e. $E_{1}$ is homothetic to $E_{2}$ ). In fact, this inequality holds for bounded measurable sets as well and it is one of the most important and deepest results in the theory of 
convex bodies. The inequality (1.1) essentially says that $[\operatorname{Vol}(\cdot)]^{1 / n}$ is a concave function with respect to Minkowski addition. The Brunn-Minkowski inequality has connections with many other inequalities including the isoperimetric inequality, Sobolev inequalities, Gagliardo-Nirenberg inequalities. Inequalities of Brunn-Minkowski type have been obtained for various other homogeneous functionals including; torsional rigidity, the first eigenvalue of the Laplacian, Newtonian capacity (for more details see the survey paper of Gardner [Ga] and the book of Schneider [Sc]). Combining results from [CS, B1, CJL], the following p-capacitary version of the Brunn-Minkowski inequality has been shown for $1<p<n$

$$
\left[\operatorname{Cap}_{p}\left(\lambda E_{1}+(1-\lambda) E_{2}\right)\right]^{\frac{1}{n-p}} \geq \lambda\left[\operatorname{Cap}_{p}\left(E_{1}\right)\right]^{\frac{1}{n-p}}+(1-\lambda)\left[\operatorname{Cap}_{p}\left(E_{2}\right)\right]^{\frac{1}{n-p}}
$$

whenever $E_{1}, E_{2}$ are compact convex sets with nonempty interiors in $\mathbb{R}^{n}$. Here $\operatorname{Cap}_{p}(\cdot)$ denotes the $p$-capacity of a set defined by

$$
\operatorname{Cap}_{p}(E)=\inf \left\{\int_{\mathbb{R}^{n}}|\nabla v|^{p} d x: v \in C_{0}^{\infty}\left(\mathbb{R}^{n}\right), v(x) \geq 1 \text { for } x \in E\right\}
$$

Moreover, equality in (1.2) holds precisely when $E_{1}$ is homothetic to $E_{2}$. In [AGHLV], the first, second, and fourth authors of this article along with Jasun Gong and Jay Hineman studied a generalized notion of $p$-capacity for $1<p<n$ associated with "A-harmonic PDEs" (see Definition 2.1) and denoted by $\operatorname{Cap}_{\mathcal{A}}(\cdot)$. It was shown in that article that the Brunn-Minkowski type inequality holds:

$$
\left[\operatorname{Cap}_{\mathcal{A}}\left(\lambda E_{1}+(1-\lambda) E_{2}\right)\right]^{\frac{1}{(n-p)}} \geq \lambda\left[\operatorname{Cap}_{\mathcal{A}}\left(E_{1}\right)\right]^{\frac{1}{(n-p)}}+(1-\lambda)\left[\operatorname{Cap}_{\mathcal{A}}\left(E_{2}\right)\right]^{\frac{1}{(n-p)}}
$$

when $1<p<n, 0 \leq \lambda \leq 1$, and $E_{1}, E_{2}$ are convex compact sets with positive $\mathcal{A}$-capacity (so $E_{1}$ or $E_{2}$ can have an empty interior). Moreover, if equality holds in the above inequality for some $E_{1}$ and $E_{2}$, then under certain regularity and structural assumptions on $\mathcal{A}$, it was concluded that these two sets are homothetic.

We note that when $p \geq n, \operatorname{Cap}_{p}(B(x, r))=0$ (see [HKM, Example 2.12]) for any $r>0$. Borell in [B2] and Colesanti and Cuoghi in [CC] considered an analogous problem when $p=n$. To describe their work, consider a convex compact $E$ with non-empty interior. It can be shown that there exists a unique $u$ which is $n$-harmonic in $\mathbb{R}^{n} \backslash E$ with continuous boundary values 0 on $\partial E$ and $u(x)=\log |x|+a+o(1)$ as $|x| \rightarrow \infty$. Now $u$ and $a \in \mathbb{R}$ are uniquely determined by $E$. If we let $\mathcal{C}_{n}(E):=e^{-a}$ then Borell in [B2] when $n=2$ and Colesanti and Cuoghi in [CC] for $p=n>2$ showed that $\mathcal{C}_{n}(\cdot)$ satisfies the Brunn-Minkowski inequality (1.2) with power $1 /(n-p)$ replaced by 1 for $n \geq 2$. In these articles, it was also shown that if equality holds for some convex compact sets $E_{1}$ and $E_{2}$ with non-empty interiors then they are homothetic. When $n=2, \mathcal{C}_{2}(\cdot)$ is often called logarithmic capacity so the authors of [CC] called $\mathcal{C}_{n}(\cdot)$ an $n$-dimensional logarithmic capacity. In the first part of this article, using a similar approach, we study a Brunn-Minkowski inequality associated with an $\mathcal{A}$-harmonic Green's function when $n \leq p<\infty$. To our knowledge this study is the first of its kind when $n<p<\infty$. 


\section{Notation And Statement of Results}

Let $n \geq 2$ and denote points in Euclidean $n$-space $\mathbb{R}^{n}$ by $y=\left(y_{1}, \ldots, y_{n}\right)$. Let $\mathbb{S}^{n-1}$ denote the unit sphere in $\mathbb{R}^{n}$. We write $e_{m}, 1 \leq m \leq n$, for the point in $\mathbb{R}^{n}$ with 1 in the $m$-th coordinate and 0 elsewhere. Let $\bar{E}, \partial E$, and $\operatorname{diam}(E)$ be the closure, boundary, and diameter of the set $E \subset \mathbb{R}^{n}$ respectively. We define $d(y, E)$ to be the distance from $y \in \mathbb{R}^{n}$ to $E$. Given two sets $E$ and $F$ in $\mathbb{R}^{n}$, let

$$
d_{\mathcal{H}}(E, F):=\max (\sup \{d(y, E): y \in F\}, \sup \{d(y, F): y \in E\})
$$

be the Hausdorff distance between the sets $E$ and $F$. Also

$$
E+F:=\{x+y: x \in E, y \in F\}
$$

is the Minkowski sum of $E$ and $F$. We write $E+x$ for $E+\{x\}$ and if $\rho$ is a non-negative real number set $\rho E=\{\rho y: y \in E\}$. Let $\langle\cdot, \cdot\rangle$ denote the standard inner product on $\mathbb{R}^{n}$ and let $|y|=\langle y, y\rangle^{1 / 2}$ be the Euclidean norm of $y$. Put

$$
B(z, r)=\left\{y \in \mathbb{R}^{n}:|z-y|<r\right\} \quad \text { whenever } z \in \mathbb{R}^{n} \text { and } r>0 .
$$

Let $d y$ denote the $n$-dimensional Lebesgue measure on $\mathbb{R}^{n}$. Let $\mathcal{H}^{\lambda}, 0<\lambda \leq n$, denote the $\lambda$-dimensional Hausdorff measure on $\mathbb{R}^{n}$ defined by

$$
\mathcal{H}^{\lambda}(E)=\lim _{\delta \rightarrow 0} \inf \left\{\sum_{j} r_{j}^{\lambda} ; E \subset \bigcup_{j} B\left(x_{j}, r_{j}\right), r_{j} \leq \delta\right\}
$$

where the infimum is taken over all possible $\delta$-covering $\left\{B\left(x_{j}, r_{j}\right)\right\}$ of $E$. If $O \subset \mathbb{R}^{n}$ is open and $1 \leq q \leq \infty$, then by $W^{1, q}(O)$ we denote the space of equivalence classes of functions $h$ with distributional gradient $\nabla h=\left(h_{y_{1}}, \ldots, h_{y_{n}}\right)$, both of which are $q$-th power integrable on $O$. Let

$$
\|h\|_{1, q}=\|h\|_{q}+\||\nabla h|\|_{q}
$$

be the norm in $W^{1, q}(O)$ where $\|\cdot\|_{q}$ is the usual Lebesgue $q$ norm of functions in the Lebesgue space $L^{q}(O)$. Next let $C_{0}^{\infty}(O)$ be the set of infinitely differentiable functions with compact support in $O$ and let $W_{0}^{1, q}(O)$ be the closure of $C_{0}^{\infty}(O)$ in the norm of $W^{1, q}(O)$. By $\nabla$. we denote the divergence operator.

Definition 2.1. Let $p, \alpha \in(1, \infty)$ and

$$
\mathcal{A}=\left(\mathcal{A}_{1}, \ldots, \mathcal{A}_{n}\right): \mathbb{R}^{n} \backslash\{0\} \rightarrow \mathbb{R}^{n},
$$

be such that $\mathcal{A}=\mathcal{A}(\eta)$ has continuous partial derivatives in $\eta_{k}$ for $k=1,2, \ldots, n$ on $\mathbb{R}^{n} \backslash\{0\}$. We say that the function $\mathcal{A}$ belongs to the class $M_{p}(\alpha)$ if the following conditions are satisfied whenever $\xi \in \mathbb{R}^{n}$ and $\eta \in \mathbb{R}^{n} \backslash\{0\}$ :

(i) Ellipticity: $\alpha^{-1}|\eta|^{p-2}|\xi|^{2} \leq \sum_{i, j=1}^{n} \frac{\partial \mathcal{A}_{i}}{\partial \eta_{j}}(\eta) \xi_{i} \xi_{j} \quad$ and $\quad \sum_{i=1}^{n}\left|\nabla \mathcal{A}_{i}(\eta)\right| \leq \alpha|\eta|^{p-2}$,

(ii) Homogeneity: $\mathcal{A}(\eta)=|\eta|^{p-1} \mathcal{A}(\eta /|\eta|)$. 
We put $\mathcal{A}(0)=0$ and note that Definition $2.1(i)$ and $(i i)$ implies

$$
\left(|\eta|+\left|\eta^{\prime}\right|\right)^{p-2}\left|\eta-\eta^{\prime}\right|^{2} \approx\left\langle\mathcal{A}(\eta)-\mathcal{A}\left(\eta^{\prime}\right), \eta-\eta^{\prime}\right\rangle
$$

whenever $\eta, \eta^{\prime} \in \mathbb{R}^{n} \backslash\{0\}$ and the proportionality constants depend only on $p, n$, and $\alpha$.

Definition 2.2. Let $p \in(1, \infty)$ and let $\mathcal{A} \in M_{p}(\alpha)$ for some $\alpha \in(1, \infty)$. Given an open set $O$ we say that $u$ is $\mathcal{A}$-harmonic in $O$ provided $u \in W^{1, p}(G)$ for each open $G$ with $\bar{G} \subset O$ and

$$
\int\langle\mathcal{A}(\nabla u(y)), \nabla \theta(y)\rangle d y=0 \quad \text { whenever } \theta \in W_{0}^{1, p}(G) .
$$

We say that $u$ is an $\mathcal{A}$-subsolution ( $\mathcal{A}$-supersolution) in $O$ if $u \in W^{1, p}(G)$ whenever $G$ is as above and (2.2) holds with $=$ replaced by $\leq(\geq)$ whenever $\theta \in W_{0}^{1, p}(G)$ with $\theta \geq 0$. As a short notation for (2.2) we write $\nabla \cdot \mathcal{A}(\nabla u)=0$ in $O$.

Remark 2.3. We remark for $O, \mathcal{A}, p, u$, as in Definition 2.2 that if $F: \mathbb{R}^{n} \rightarrow \mathbb{R}^{n}$ is the composition of a translation and a dilation then

$$
\hat{u}(z)=u(F(z)) \text { is } \mathcal{A} \text {-harmonic in } F^{-1}(O) \text {. }
$$

Moreover, if $\tilde{F}: \mathbb{R}^{n} \rightarrow \mathbb{R}^{n}$ is the composition of a translation, a dilation, and a rotation then

$$
\tilde{u}(z)=u(\tilde{F}(z)) \text { is } \tilde{\mathcal{A}} \text {-harmonic in } \tilde{F}^{-1}(O) \text { for some } \tilde{\mathcal{A}} \in M_{p}(\alpha) \text {. }
$$

We note that $\mathcal{A}$-harmonic PDEs have been studied in [HKM]. Also dimensional properties of the Radon measure associated with a positive $\mathcal{A}$-harmonic function $u$, vanishing on a portion of the boundary of $O$, have been studied in [A, ALV12, ALV, LN, AGHLV], see also [LLN, LN4].

In this article, we often assume that $E \subset \mathbb{R}^{n}$ is a convex compact set usually containing at least two points or equivalently having positive $\mathcal{H}^{1}$ measure. Note that when $p=2$ and $\mathcal{A}(\eta)=\left(\eta_{1}, \ldots, \eta_{n}\right), \eta \in \mathbb{R}^{n} \backslash\{0\}$, then one gets Laplace's equation in $\mathbb{R}^{n}$. If we additionally let $n=2$ and $\Omega=\mathbb{R}^{2} \backslash E$, then the logarithmic capacity of $E$ can be defined through the behavior of the Green's function near $\infty$. That is, the Green's function $u(\cdot, \infty)$ with pole at $\infty$ has continuous boundary values zero on $\partial E$, and has a representation $u(z, \infty)=\log |z|+H(z, \infty)$ in $\Omega$ where $H(z, \infty)$ is harmonic, bounded, and continuous at $\infty$. The quantity $H(\infty, \infty)=a$ is known as the Robin's constant for $E$. Thus $u(z, \infty)=\log |z|+a+o(1)$ as $|z| \rightarrow \infty$. Classically the logarithmic capacity of $E$ in the plane is defined by $\operatorname{Cap}_{2}(E)=e^{-a}$. More about logarithmic capacity in the plane can be found in [GM, Chapter III] and in [La, Page 167].

When $\mathcal{A}(\eta)=|\eta|^{p-2} \eta$ for $\eta \in \mathbb{R}^{n} \backslash\{0\}$ and $1<p<\infty$ then one gets the pLaplace equation $\nabla \cdot\left(|\nabla u|^{p-2} \nabla u\right)=0$ and solutions are called $p$-harmonic functions. In $\mathbb{R}^{n}, n \geq 2, \log |x|$ is an $n$-harmonic function and $|x|^{\frac{p-n}{p-1}}$ is a $p$-harmonic function in $\mathbb{R}^{n} \backslash\{0\}$ when $1<p \neq n<\infty$. As in the planar case, given a convex compact set $E \subset \mathbb{R}^{n}$ with non-empty interior, there exists a unique $p$-harmonic function $u$ 
such that $\nabla \cdot\left(|\nabla u|^{p-2} \nabla u\right)=0$ in $\mathbb{R}^{n} \backslash E$ with continuous boundary values 0 on $\partial E$ satisfying

$$
u(x)=\left\{\begin{array}{l}
\log |x|+a+o(1) \quad \text { when } p=n, \\
|x|^{\frac{p-n}{p-1}}+a+o(1) \quad \text { when } n<p<\infty,
\end{array} \quad \text { as }|x| \rightarrow \infty .\right.
$$

Here $a \in \mathbb{R}$ when $p=n$ and $a<0$ when $n<p<\infty$. So based on the classical function theory, $p=2, n=2$, case, a natural definition of $\mathcal{C}_{p}(E)$ is $\mathcal{C}_{p}(E)=e^{-a}$ when $p=n$ and $\mathcal{C}_{p}(E)=(-a)^{p-1}$ when $n<p<\infty$. It can be shown that $u$ and $a$ are uniquely determined by $E$. Moreover, $t \mapsto \mathcal{C}_{p}(t E)$ for $t \in(0, \infty)$ is homogeneous of degree 1 function when $p=n$ while this function is homogeneous of degree $p-n$ when $n<p<\infty$.

Using the above approach but a more PDE inspired normalization, we will define $\mathcal{C}_{\mathcal{A}}(E)$, whenever $n \leq p<\infty$, and $E$ contains at least two points when $p=n$ or is nonempty when $p>n$. We will show in $\S 4$ that there exists a unique $u$, satisfying

$$
\left\{\begin{array}{l}
\nabla \cdot \mathcal{A}(\nabla u)=0 \text { in } \mathbb{R}^{n} \backslash E \\
u=0 \text { on } \partial E \\
u(x)=F(x)+a+o(1) \text { as }|x| \rightarrow \infty
\end{array}\right.
$$

where $F(x)$ is the so called fundamental solution to an $\mathcal{A}$-harmonic PDE with pole at infinity (see Lemma 4.2 and Lemma 4.4). Here $F(x) \approx \log |x|$, when $p=n$ and $F(x) \approx|x|^{\frac{p-n}{p-1}}$ when $n<p<\infty$ as $x \rightarrow \infty$. Given $E$ as above we call $u$ the $\mathcal{A}$ harmonic Green's function for $\mathbb{R}^{n} \backslash E$ with pole at $\infty$. We will also see in $\S 5$ that $u$ and $a$ are uniquely determined by $E$. Thereupon, we define

$$
\mathcal{C}_{\mathcal{A}}(E)= \begin{cases}e^{-a / \gamma} & \text { when } p=n \\ (-a)^{p-1} & \text { when } n<p<\infty\end{cases}
$$

Here $\gamma$ is a non-negative constant (see Lemma 4.4). With this notation we prove that $\mathcal{C}_{\mathcal{A}}(\cdot)$ satisfies the following Brunn-Minkowski type inequality when $n \leq p<\infty$. In particular, our first main result is

Theorem A. Let $E_{1}$ and $E_{2}$ be compact convex sets in $\mathbb{R}^{n}, n \geq 2$. Assume that both sets contain at least two points when $p=n$ and that both sets are nonempty when $p>n$. If $\lambda \in[0,1]$ and if $p=n$ then

$$
\mathcal{C}_{\mathcal{A}}\left(\lambda E_{1}+(1-\lambda) E_{2}\right) \geq \lambda \mathcal{C}_{\mathcal{A}}\left(E_{1}\right)+(1-\lambda) \mathcal{C}_{\mathcal{A}}\left(E_{2}\right)
$$

While if $n<p<\infty$ then

$$
\left[\mathcal{C}_{\mathcal{A}}\left(\lambda E_{1}+(1-\lambda) E_{2}\right)\right]^{\frac{1}{p-n}} \geq \lambda \mathcal{C}_{\mathcal{A}}\left(E_{1}\right)^{\frac{1}{p-n}}+(1-\lambda) \mathcal{C}_{\mathcal{A}}\left(E_{2}\right)^{\frac{1}{p-n}}
$$


If equality holds in (2.4) or in (2.5) and $\mathcal{A}$ satisfies

(i) There exists $1 \leq \Lambda<\infty$ such that $\left|\frac{\partial \mathcal{A}_{i}}{\partial \eta_{j}}(\eta)-\frac{\partial \mathcal{A}_{i}}{\partial \eta_{j}^{\prime}}\left(\eta^{\prime}\right)\right| \leq \Lambda\left|\eta-\eta^{\prime}\right||\eta|^{p-3}$ whenever $0<\frac{1}{2}|\eta| \leq\left|\eta^{\prime}\right| \leq 2|\eta|$ and $1 \leq i \leq n$,

(ii) $\mathcal{A}_{i}(\eta)=\frac{\partial f}{\partial \eta_{i}}$ for $1 \leq i \leq n$ where $f(t \eta)=t^{p} f(\eta)$ when $t>0$ and $\eta \in \mathbb{R}^{n} \backslash\{0\}$,

then $E_{2}$ is a translation and dilation of $E_{1}$ provided that both sets contain at least two points.

We first remark that the work in [CC, B2] corresponds to the case $p=n$ and $\mathcal{A}(\eta)=|\eta|^{n-2} \eta$, whereas in Theorem A we consider all possible values of $p, n \leq p<\infty$ and general $\mathcal{A} \in M_{p}(\alpha)$ for $\alpha \in(1, \infty)$. Second, in [CC, B2], the convex compact sets $E_{1}$ and $E_{2}$ are required to have interiors, unlike our "bare bones" assumption on $E_{1}$ and $E_{2}$. We consider it a very interesting question as to whether (2.6) is needed in Theorem A. Finally, Theorem A will be used to prove uniqueness in the Minkowski type problem associated with an $\mathcal{A}$-harmonic Green's function for the complement of a compact convex set when $p \geq n$ (see $\S 7$ ).

\section{Basic estimates for $\mathcal{A}$-Harmonic functions}

In this section we state some fundamental estimates for $\mathcal{A}$-harmonic functions. Concerning constants, unless otherwise stated, in this section, and throughout the paper, $c$ will denote a positive constant $\geq 1$, not necessarily the same at each occurrence, depending at most on $p, n, \alpha, \Lambda$ which sometimes we refer to as depending on the data. In general, $c\left(a_{1}, \ldots, a_{m}\right)$ denotes a positive constant $\geq 1$, which may depend at most on the data and $a_{1}, \ldots, a_{m}$, not necessarily the same at each occurrence. If $B \approx C$ then $B / C$ is bounded from above and below by constants which, unless otherwise stated, depend at most on the data. Moreover, we let $\max _{F} \tilde{u}, \min _{F} \tilde{u}$ be the essential supremum and infimum of $\tilde{u}$ on $F$ whenever $F \subset \mathbb{R}^{n}$ and $\tilde{u}$ is defined on $F$. Finally in this section, unless otherwise stated, we assume that $1<p<\infty$. We shall start with a definition of thickness and fatness of a closed set

Definition 3.1 (Thickness). Given $\lambda>0$ and $r_{0}>0$, a closed set $E$ is called $\left(r_{0}, \lambda\right)$-thick if there exists $c>0$ such that

$$
\mathcal{H}_{\infty}^{\lambda}(E \cap \bar{B}(w, r)) \geq c r^{\lambda} \quad \text { for all } \quad 0<r \leq r_{0} \quad \text { and } \quad w \in E .
$$

Here $\mathcal{H}_{\infty}^{\lambda}$ denotes the Hausdorff content of a set (take the infimum in the definition of $\mathcal{H}^{\lambda}$ over all possible covering of the set). The classical definition of the $p$-capacity of a compact set $E$ inside a bounded domain $D$ is defined by

$$
\operatorname{Cap}_{p}(E, D)=\inf \left\{\int_{D}|\nabla v|^{p} d x: v \in C_{0}^{\infty}(D), v(x) \geq 1 \text { for } x \in E\right\} .
$$

Note that when $D=\mathbb{R}^{n}$ one has $\operatorname{Cap}_{p}\left(E, \mathbb{R}^{n}\right)=\operatorname{Cap}_{p}(E)$ as defined earlier. 
Definition 3.2 (Fatness). A closed set $E \subset \mathbb{R}^{n}$ is called uniformly $\left(r_{0}, p\right)$-fat if

$$
\frac{C_{a p}(E \cap \bar{B}(w, r), B(w, 2 r))}{\operatorname{Cap}_{p}(\bar{B}(w, r), B(w, 2 r))} \geq c
$$

for $0<r \leq r_{0}$ and all $w \in E$.

We note from [HKM, Example 2.12] that for $n<p<\infty$,

$$
\operatorname{Cap}_{p}\left(\left\{x_{0}\right\}, B\left(x_{0}, 2 r\right)\right) \approx \operatorname{Cap}_{p}\left(\bar{B}\left(x_{0}, r\right), B\left(x_{0}, 2 r\right)\right) \approx r^{n-p} .
$$

Thus if $p>n$ and $0<r_{0}<\infty$ then any nonempty closed set is uniformly $\left(r_{0}, p\right)$-fat. On the other hand, if $1<p \leq n$, it follows essentially from Frostman's lemma that if a closed set $E$ is $\left(r_{0}, \lambda\right)$-thick for some $r_{0}$ and $\lambda>n-p$ then $E$ is uniformly $\left(r_{0}, p\right)$ fat (see [AH, Corollary 5.1.14]). Thus a compact convex set containing at least two points is uniformly $\left(r_{0}, n\right)$-fat with capacitary ratio (i.e, $c$ in Definition 3.2) depending only on $n$ when $r_{0}=\operatorname{diam}(E)$. Finally, uniform $\left(r_{0}, p\right)$-fatness for some $r_{0}>0$, is a sufficient condition for solvability of the Dirichlet problem for $\mathcal{A}$-harmonic PDEs in a bounded domain $\Omega$ in the sense that if every point $w \in \mathbb{R}^{n} \backslash \Omega$ is uniformly $\left(r_{0}, p\right)$-fat, then

$$
\int_{0}^{r_{0}}\left[\frac{\operatorname{Cap}_{p}\left(\left(\mathbb{R}^{n} \backslash \Omega\right) \cap \bar{B}(w, r), B(w, 2 r)\right)}{\operatorname{Cap}_{p}(\bar{B}(w, r), B(w, 2 r))}\right]^{\frac{1}{(p-1)}} \frac{d r}{r}=\infty .
$$

That is, uniform $\left(r_{0}, p\right)$-fatness implies Wiener regularity (see [HKM, Theorem 6.33]). Next we state some basic estimates for $\mathcal{A}$-harmonic functions.

Lemma 3.3. Given $p, 1<p<\infty$, assume that $\tilde{\mathcal{A}} \in M_{p}(\alpha)$ for some $\alpha \in(1, \infty)$ in $\mathbb{R}^{n}, n \geq 2$. Let $\tilde{u}$ be a $\tilde{\mathcal{A}}$-harmonic function in $B(w, 4 r)$ for some $r>0$. Then

$$
\begin{aligned}
& \text { (i) } r^{p-n} \int_{B(w, r / 2)}|\nabla \tilde{u}|^{p} d y \leq c\left(\max _{B(w, r)}|\tilde{u}|\right)^{p} \leq c^{2} r^{-n} \int_{B(w, 2 r)}|\tilde{u}|^{p} d x, \\
& \text { (ii) If } \tilde{u} \geq 0 \text { in } B(w, 2 r) \text { then } \max _{B(w, r)} \tilde{u} \leq c \min _{B(w, r)} \tilde{u} .
\end{aligned}
$$

Furthermore, there exists $\tilde{\sigma}=\tilde{\sigma}(p, n, \alpha) \in(0,1)$ such that

$$
\text { (iii) }|\tilde{u}(x)-\tilde{u}(y)| \leq c\left(\frac{|x-y|}{r}\right)^{\tilde{\sigma}} \max _{B(w, 2 r)}|\tilde{u}|
$$

whenever $x, y \in B(w, r)$.

Proof. A proof of this lemma can be found in [S].

Lemma 3.4. Let $p, n, \tilde{\mathcal{A}}, \alpha, w, r, \tilde{u}$ be as in Lemma 3.3. Then $\tilde{u}$ has a representative locally in $W^{1, p}(B(w, 4 r)$ ), with Hölder continuous partial derivatives in $B(w, 4 r)$ (also denoted $\tilde{u})$. Moreover, there exist $\tilde{\beta} \in(0,1]$ and $c \geq 1$, depending only on $p$, $n$, and 
$\alpha$, such that if $x, y \in B(w, r)$, then

$$
\begin{aligned}
& (\hat{a}) c^{-1}|\nabla \tilde{u}(x)-\nabla \tilde{u}(y)| \leq(|x-y| / r)^{\tilde{\beta}} \max _{B(w, r)}|\nabla \tilde{u}| \leq c r^{-1}(|x-y| / r)^{\tilde{\beta}} \tilde{u}(w), \\
& (\hat{b}) \int_{B(w, r)} \sum_{i, j=1}^{n}|\nabla \tilde{u}|^{p-2}\left|\tilde{u}_{x_{i} x_{j}}\right|^{2} d y \leq c r^{(n-p-2)} \tilde{u}(w) .
\end{aligned}
$$

If

$$
\gamma r^{-1} \tilde{u} \leq|\nabla \tilde{u}| \leq \gamma^{-1} r^{-1} \tilde{u} \quad \text { on } \quad B(w, 2 r)
$$

for some $\gamma \in(0,1)$ and (2.6) $(i)$ holds then $\tilde{u}$ has Hölder continuous second partial derivatives in $B(w, r)$ and there exist $\tilde{\theta} \in(0,1)$ and $\bar{c} \geq 1$, depending only on the data and $\gamma$, such that

$$
\begin{aligned}
{\left[\sum_{i, j=1}^{n}\left(\tilde{u}_{x_{i} x_{j}}(x)-\tilde{u}_{y_{i} y_{j}}(y)\right)^{2}\right]^{1 / 2} } & \leq \bar{c}(|x-y| / r)^{\tilde{\theta}} \max _{B(w, r)}\left(\sum_{i, j=1}^{n}\left|\tilde{u}_{x_{i} x_{j}}\right|\right) \\
& \leq \bar{c}^{2} r^{-n / 2}(|x-y| / r)^{\tilde{\theta}}\left(\sum_{i, j=1}^{n} \int_{B(w, 2 r)} \tilde{u}_{x_{i} x_{j}}^{2} d x\right)^{1 / 2} \\
& \leq \bar{c}^{3} r^{-2}(|x-y| / r)^{\tilde{\theta}} \tilde{u}(w)
\end{aligned}
$$

whenever $x, y \in B(w, r / 2)$.

Proof. A proof of (3.2) can be found in [T]. Also, (3.3) follows from (3.2), the added assumptions, and Schauder type estimates (see [GT]).

Lemma 3.5. Fix $p$ with $1<p<\infty$ and assume that $\tilde{\mathcal{A}} \in M_{p}(\alpha)$ for some $\alpha \in(1, \infty)$. Let $\tilde{E} \subset B(0, R)$, for some $R>0$, be a uniformly $\left(r_{0}, p\right)$-fat compact convex set where $r_{0}=\operatorname{diam}(\tilde{E})$. Let $\zeta \in C_{0}^{\infty}(B(0,2 R))$ with $\zeta \equiv 1$ on $B(0, R)$. If $0 \leq \tilde{u}$ is $\tilde{\mathcal{A}}$-harmonic in $B(0,4 R) \backslash \tilde{E}$, and $\tilde{u} \zeta \in W_{0}^{1, p}(B(0,4 R) \backslash \tilde{E})$, then $\tilde{u}$ has a continuous extension to $B(0,4 R)$ obtained by putting $\tilde{u} \equiv 0$ on $\tilde{E}$. Moreover, if $0<r<R$ and $w \in \tilde{E}$ then

$$
\text { (i) } r^{p-n} \int_{B(w, r)}|\nabla \tilde{u}|^{p} d y \leq c\left(\max _{B(w, 2 r)} \tilde{u}\right)^{p} .
$$

Furthermore, there exists $\hat{\sigma} \in(0,1)$, depending on $p, n, \alpha$, and the uniform $\left(r_{0}, p\right)$ fatness constant for $\tilde{E}$, such that

$$
\text { (ii) }|\tilde{u}(x)-\tilde{u}(y)| \leq c\left(\frac{|x-y|}{r}\right)^{\hat{\sigma}} \max _{B(w, 2 r)} \tilde{u}
$$

whenever $x, y \in B(w, r)$ and $0<r<\operatorname{diam}(\tilde{E})$.

Proof. Here $(i)$ is a standard Caccioppoli inequality and ( $i i)$ for $y \in \tilde{E}$ follows from uniform $\left(r_{0}, p\right)$-fatness of $\tilde{E}$ and essentially Theorem 6.18 in [HKM]. Combining this fact with (3.1) (iii) we obtain (ii). 
Remark 3.6. As an application of Lemma 3.5 observe from the remarks following Definitions 3.1 and 3.2 that if $p>n$ then Lemma 3.5 is valid for any compact set $\tilde{E} \subset B(0, R)$ with $\operatorname{diam}(\tilde{E})$ in (3.4) (ii) replaced by $R$. If $p=n$ and $\tilde{E}$ is convex, containing at least two points, then $\tilde{E}$ contains a line segment so is $\left(r_{0}, 1\right)$-thick and thus uniformly $\left(r_{0}, n\right)$-fat. Hence Lemma 3.5 applies in this situation as well.

Lemma 3.7. Let $\tilde{\mathcal{A}}, p, n, \alpha, \tilde{E}, r_{0}, R, \tilde{u}$ be as in Lemma 3.5. Then there exists a unique finite positive Borel measure $\tilde{\mu}$ with support contained in $\tilde{E}$ such that

$$
\text { (i) } \int\langle\tilde{\mathcal{A}}(\nabla \tilde{u}(y)), \nabla \phi(y)\rangle d y=-\int \phi d \tilde{\mu} \quad \text { whenever } \phi \in C_{0}^{\infty}(B(0,2 R)) \text {. }
$$

Moreover, there exists $c \geq 1$, with the same dependence as in Lemma 3.5 such that

$$
\text { (ii) } c^{-1} r^{p-n} \tilde{\mu}(B(w, r)) \leq \max _{B(w, 2 r)} \tilde{u}^{p-1} \leq c r^{p-n} \tilde{\mu}(B(w, 4 r))
$$

whenever $0<r \leq r_{0}$ and $w \in \tilde{E}$.

Proof. For the proof of $(i)$ see [HKM, Theorem 21.2] or [KZ]. The left-hand inequality in (ii), follows from $(i)$ in $(2.1)$ with $\eta^{\prime}=(0, \ldots, 0)$, and Hölder's inequality, using a test function, $\phi$, with $\phi \equiv 1$ on $\bar{B}(w, r)$. The proof of the right-hand inequality in (3.5) (ii) follows from [EL] or [KZ].

\section{Existence AND Uniqueness of a fUNDAMENTAL SOlUtion}

Let $p$ and $\alpha$ be fixed with $2 \leq n \leq p<\infty$ and $\alpha \in(1, \infty)$. In this section, for $\mathcal{A} \in M_{p}(\alpha)$, we will show existence and uniqueness of a fundamental solution to $\mathcal{A}$-harmonic PDE. The cases $p>n$ and $p=n$ require different proofs and slightly different definitions. We begin with the more difficult case $p=n$.

\subsection{Existence and uniqueness for $p=n$.}

Definition 4.1. If $p=n$ we say that $F$ is a fundamental solution to $\nabla \cdot \mathcal{A}(\nabla F)=0$ in $\mathbb{R}^{n}$ with pole at 0 if

$$
\begin{aligned}
& F \text { is } \mathcal{A} \text {-harmonic in } \mathbb{R}^{n} \backslash\{0\}, F \in W_{\text {loc }}^{1, l}\left(\mathbb{R}^{n}\right) \text { for } 1<l<n, \\
& \text { and }|F(x)|=O(\log |x|) \text { in a neighborhood of } \infty \text {. } \\
& \quad \int\langle\mathcal{A}(\nabla F(z)), \nabla \theta(z)\rangle d z=-\theta(0) \quad \text { whenever } \theta \in C_{0}^{\infty}\left(\mathbb{R}^{n}\right) \text {. }
\end{aligned}
$$

In order to show existence and uniqueness of $F$ we require several preliminary lemmas. Fix $R$ with $2<R<\infty$, and let $v=v(\cdot, R)$ be the $\mathcal{A}$-harmonic function in $B(0, R) \backslash \bar{B}(0,1)$ with continuous boundary values 0 on $\partial B(0,1)$ and $\log R$ on $\partial B(0, R)$. Extend $v$ to $B(0,1)$ by putting $v \equiv 0$ on $B(0,1)$. Let $\nu$ be the positive Borel measure as in Lemma 3.7 with support contained on $\partial B(0,1)$ associated to $v$. We claim that 
Lemma 4.2. For $v$ and $\nu$ as above we have

(a) $\nu(\bar{B}(0,1)) \approx 1$,

(b) $\quad c^{-1} \leq\langle\nabla v(x), x\rangle \leq|x||\nabla v| \leq c \quad$ whenever $x \in B(0, R) \backslash B(0,1)$,

(c) $v(x) \approx \log |x|$ whenever $x \in B(0, R) \backslash B(0,1)$

where ratio constants depend only on $n$ and $\alpha$.

Proof. To prove this inequality let $w(x)=\log |x|$, when $x \in B(0, R)$, and note that $w$ is $n$-harmonic in $B(0, R) \backslash \bar{B}(0,1)$ with continuous boundary values and $v \equiv w$ on $\partial[B(0, R) \backslash B(0,1)]$. Then

$$
\nu(\bar{B}(0,1)) \log R=\int_{B(0, R) \backslash \bar{B}(0,1)}\langle\mathcal{A}(\nabla v), \nabla v\rangle d x \approx \int_{B(0, R) \backslash \bar{B}(0,1)}|\nabla w|^{n} d x \approx \log R .
$$

where all ratio constants depend only on $n$ and $\alpha$. The left-hand inequality in (4.3) follows from the definition of $\nu$, using $\max (\log R-v, 0)$ as a test function. That is, as in Lemma 3.7, we first have

$$
\begin{aligned}
\left.-\int\langle\mathcal{A}(\nabla v), \nabla v)\right\rangle d x & =\int\langle\mathcal{A}(\nabla v), \nabla(\log R-v)\rangle d x \\
& =-\int_{\partial B(0,1)} \log R d \nu=-\log R \nu(\bar{B}(0,1)) .
\end{aligned}
$$

The middle inequality in (4.3) follows from Hölder's inequality after using $v-w$ as a test function in the definition of $\mathcal{A}$ and $n$-harmonic functions using $v, w$. Indeed, since $v$ is $\mathcal{A}$-harmonic in $B(0, R) \backslash \bar{B}(0,1)$

$$
0=\int\langle\mathcal{A}(\nabla v), \nabla(v-w)\rangle d x=\int\langle\mathcal{A}(\nabla v), \nabla v\rangle d x-\int\langle\mathcal{A}(\nabla v), \nabla w\rangle d x .
$$

Using the structural assumptions on $\mathcal{A}$ and Hölder's inequality with $\epsilon$ 's to estimate the second integral on the right in the above equality, it follows that

$$
\int|\nabla v|^{n} d x \leq \alpha \int\langle\mathcal{A}(\nabla v), \nabla v\rangle d x=\alpha \int\langle\mathcal{A}(\nabla v), \nabla w\rangle d x \leq c \int|\nabla w|^{n} d x
$$

where $c$ depends only on $n$ and $\alpha$. A similar argument using $n$-harmonicity of $w$ and $v-w$ as a test function gives the reverse inequality. Thus (4.2) (a) is valid. To prove $(4.2)(b)$ we note that $\partial B(0,1)$ is uniformly $(1, n)$-fat in the sense of $n$-capacity defined relative to $B(0,4)$. It follows from Lemma $3.7(i i)$ and Harnack's inequality for $\mathcal{A}$-harmonic functions that

$$
1 \approx \nu(\bar{B}(0,1))^{1 /(n-1)} \approx \max _{\partial B(0,2)} v .
$$

Similarly, if $\tau$ is the measure with support contained on $\partial B(0, R)$ associated with $\log R-v$ as in Lemma 3.7 and $\tilde{\mathcal{A}}(\eta)=-\mathcal{A}(-\eta)$ then

$$
1 \approx \nu(\bar{B}(0,1))^{1 /(n-1)}=\tau(\bar{B}(0, R))^{1 /(n-1)} \approx \max _{\partial B(0, R / 2)}(\log R-v) .
$$


We now argue as in the proof of Lemma 4.3 in [AGHLV]. For fixed $1<\lambda<101 / 100$, we claim that

$$
k(x):=\frac{v(\lambda x)-v(x)}{\lambda-1} \geq c^{-1} \quad \text { whenever } x \in B(0, R / \lambda) \backslash B(0,1)
$$

where $c$ depends only on $n$ and the structure constants for $\mathcal{A}$. To prove (4.6) let

$$
\phi_{1}(x)=\frac{e^{N|x|^{2}}-e^{N}}{e^{64 N}-e^{N}} \quad \text { and } \quad \phi_{2}(x)=\frac{e^{-N|x|^{2}}-e^{-64 N}}{e^{-N}-e^{-64 N}} .
$$

Notice that $\phi_{1}=0$ on $\partial B(0,1)$ and $\phi_{1}=1$ on $\partial B(0,8)$. Similarly, $\phi_{2}=1$ on $\partial B(0,1)$ and $\phi_{2}=0$ on $\partial B(0,8)$. Note also that at points in $B(0,8) \backslash \bar{B}(0,1)$ where $\nabla v \neq 0$, these functions are subsolutions to the second order non-divergence form PDE for $v$ corresponding to $\mathcal{A}$-harmonicity when $N \geq 1$ is sufficiently large depending only on the data (see [AGHLV, Section 7.1]). Using these notes and arguing as in [AGHLV, Section 7.1] we see there exists $N, c_{1} \geq 1$, depending only on the structure constants for $\mathcal{A}, \alpha$, and $n$ such that

$$
c_{1}^{3}(|y|-1) \geq c_{1}^{2}\left[1-\phi_{2}(y)\right] \geq c_{1} v(y) \geq \phi_{1}(y) \geq c_{1}^{-1}(|y|-1)
$$

when $y \in B(0,8) \backslash \bar{B}(0,1)$. From the lower bound for $v$ in (4.8) we find that (4.6) is valid when $x \in \partial B(0,1)$ for $c$ suitably large. Similarly

$$
c_{3}(1-|y| / R) \geq c_{1}^{2}\left[1-\phi_{1}(8 y / R)\right] \geq c_{1}(\log R-v(y)) \geq \phi_{2}(8 y / R) \geq c_{1}^{-1}(1-|y| / R)
$$

when $y \in B(0, R) \backslash B(0, R / 8)$. From the lower bound for $\log R-v$ in (4.9) we deduce that (4.6) holds for $x \in \partial B(0, R / \lambda)$. From the boundary maximum principle for $\mathcal{A}$ harmonic functions it follows that (4.6) is true. Letting $\lambda \rightarrow 1$ and using the chain rule we obtain the left-hand inequality in (4.2) (b). The right-hand inequality in (4.2) (b) for $y \in B(0,8) \backslash B(0,1)$ follows from (3.5) (ii), (4.5), Lemma $3.4(\hat{a})$ with $v=u$ and the estimate for $v$ from above in (4.8). Likewise using $\log R-v=u$ in these displays and the estimate from above for $\log R-v$ in (4.9) we arrive at the right-hand inequality in (4.2) (b) for $y \in B(0, R) \backslash B(0, R / 8)$. To handle other values of $y$ we need some notation. Given a real valued continuous function $q$ defined on $\partial B(0, s)$, set

$$
m(s, q)=\min _{x \in \partial B(0, s)} q(x) \text { and } \quad M(s, q)=\max _{x \in \partial B(0, s)} q(x) .
$$

If $2<t<R / 4$ we note that

$$
E=\{x ; v(x) \leq m(t, v)\} \subset \bar{B}(0, t)
$$

is connected and has diameter at least $t$. Also $\max (v(x)-m(t, v), 0) \geq 0$ is $\mathcal{A}$ harmonic in $B(0, R) \backslash E$ and $\equiv 0$ on $E$. Let $\beta=\beta_{t}$ be the positive Borel measure with support contained in $E$ corresponding to $\max (v(x)-m(t, v), 0)$ as in Lemma 3.7. As in Lemma 4.2 of $[\mathrm{AGHLV}]$ we see that $\beta(E)=\nu(\bar{B}(0,1))$. So once again from 
Lemma 3.7 we have

$$
\begin{aligned}
1 & \approx \nu(\bar{B}(0,1))^{1 /(n-1)}=\beta(E)^{1 /(n-1)} \approx \max _{\partial B(0,2 t)}[\max (v(x)-m(t, v), 0)] \\
& =M(2 t, v)-m(t, v)
\end{aligned}
$$

for $2<t<R / 4$. Now from Lemmas 3.3-3.5 with $u$ replaced by $v-m(t, v)$ and $(4.10)$ we see that

$$
M(3 t / 2,|\nabla v|) \leq c t^{-1} .
$$

All constants in (4.10)-(4.11) depend only on the data. Combining (4.10)-(4.11) we get the right-hand inequality in (4.2) (b) when $4 \leq|y| \leq R / 4$. Thus (4.2)(b) is valid. Now (4.2)(c) follows from (4.2)(b) and integration.

To avoid confusion we temporarily write $v(\cdot, R)$ and $\nu(\cdot, R)$, for $v$ and $\nu$ in Lemma 4.2. Using Ascoli's theorem and Lemmas 3.3-3.5 for $\mathcal{A}$-harmonic functions we see as $m \rightarrow \infty$ that a sub-sequence of $\{v(\cdot, m)\}_{m=8}^{\infty}$ converges uniformly on compact subsets of $\mathbb{R}^{n}$ to a locally Hölder continuous function $\hat{V} \geq 0$ which is $\mathcal{A}$-harmonic in $\mathbb{R}^{n} \backslash \bar{B}(0,1)$ and $\hat{V} \equiv 0$ on $\bar{B}(0,1)$. Let $\hat{\Theta}$ be the measure corresponding to $\hat{V}$ as in Lemma 3.7 and put

$$
\Theta(\cdot)=\frac{\hat{\Theta}(\cdot)}{\hat{\Theta}(\bar{B}(0,1))} \quad \text { and } \quad V(\cdot)=\frac{\hat{V}(\cdot)}{[\hat{\Theta}(\bar{B}(0,1))]^{1 /(n-1)}} .
$$

Then Lemma 4.2 is valid with $v$ and $\nu$ replaced by $V$ and $\Theta$ in $\mathbb{R}^{n} \backslash B(0,1)$, and $\Theta(\bar{B}(0,1))=1$. Next we prove

Lemma 4.3. For $V$ and $\Theta$ as above there exist $R_{0} \geq 4, b \in C^{1, \sigma}(B(0,2) \backslash \bar{B}(0,1 / 2))$, $\tilde{\alpha} \in(0,1), c_{+} \geq 1$, and $0<\gamma \leq c_{+}$where $\tilde{\alpha}, \sigma, c_{+}$, depend only on the data, satisfying

$$
|V(x)-\gamma \log | x|-b(x /|x|)| \leq c_{+}\left(R_{0} /|x|\right)^{\tilde{\alpha}}
$$

whenever $|x|=R \geq R_{0} \geq 4$.

Proof. We first note that $h(x):=\langle x, \nabla V(x)\rangle$ is a weak solution to

$$
L h=\sum_{i, j=1}^{n} \frac{\partial}{\partial x_{i}}\left(\mathfrak{b}_{i j}(x) h_{x_{j}}\right)=0
$$

whenever $x \in \mathbb{R}^{n} \backslash \bar{B}(0,1)$ where

$$
\mathfrak{b}_{i j}(x)=\frac{\partial \mathcal{A}_{i}(\nabla V(x))}{\partial \eta_{j}}
$$

for almost every $x \in \mathbb{R}^{n} \backslash \bar{B}(0,1)$. From (4.14) and the structural assumptions on $\mathcal{A}$ in Definition 2.1 it follows that

$$
\sum_{i, j=1}^{n} \mathfrak{b}_{i j}(x) \xi_{i} \xi_{j} \approx|\nabla V(x)|^{n-2}|\xi|^{2} \quad \text { whenever } \xi \in \mathbb{R}^{n} \backslash\{0\}
$$


where ratio constants depend only on the data. From (4.13)-(4.15) and (4.2) (b) we see for $r \geq 8$ that $h$ is a bounded positive weak solution to a uniformly elliptic PDE in $B(0,2 r) \backslash B(0, r / 2)$ with bounded measurable coefficients.

We observe that $m(r, h)$ as a function of $r$ is either non-decreasing on $(1, \infty)$ or ultimately non-increasing in the sense that there exists $t_{1}>1$ with $m(\cdot, h)$ nonincreasing on $\left[t_{1}, \infty\right)$. Also either $M(r, h)$ is non-increasing on $(1, \infty)$ or ultimately non-decreasing on $(1, \infty)$ in the sense that there exists $t_{1}>1$ with $M(\cdot, h)$ nondecreasing on $\left[t_{1}, \infty\right)$. Both statements follow from the maximum-minimum principles for $\mathcal{A}$-harmonic functions. Thus there exist constants $\beta$ and $\gamma$ such that

$$
0<\beta:=\lim _{r \rightarrow \infty} m(r, h) \leq \lim _{r \rightarrow \infty} M(r, h)=: \gamma<\infty
$$

thanks to Lemma 4.2. Then $h-\min (m(r / 2, h), m(2 r, h))$ is non-negative in $B(0,2 r) \backslash$ $B(0, r / 2)$ so by Harnack's inequality for uniformly elliptic PDE in (4.13) we have,

$$
M(r, h)-\min (m(r / 2, h), m(2 r, h)) \leq c[m(r, h)-\min (m(r / 2, h), m(2 r, h))],
$$

$c$ depending only on the data. Letting $r \rightarrow \infty$ in (4.16) we obtain that

$$
\gamma=\beta<\infty \text {. }
$$

To get the estimate in (4.12) first assume that $M(\cdot, h)$, is non-decreasing on $\left[t_{1}, \infty\right)$ for some $t_{1} \geq 1$. Then from (4.17) it follows that $m(\cdot, h)$ is also non-decreasing on $\left[t_{1}, \infty\right)$. In this case we show the existence of $t_{2} \geq t_{1}$ with

$$
h \equiv \gamma \quad \text { on } \mathbb{R}^{n} \backslash B\left(0, t_{2}\right) .
$$

From (4.17) and Harnack's inequality for $L$ in (4.13) applied to $h-m(t, h)$ it follows that either $m(t, h)=\gamma$ for some $t>t_{1}$ in which case we put $t_{2}=t$ and observe that (4.18) is valid or there exists $t_{2}>t_{1}$ with

$$
\gamma>m(t, h)>M\left(t_{1}, h\right) \text { for } t \geq t_{2} .
$$

Assuming (4.19) we claim that for $\mathcal{H}^{1}$-almost every $\tau \in\left[m\left(t_{2}, h\right), \gamma\right]$,

$$
I(\tau):=\int_{\left\{x \in \mathbb{R}^{n} \backslash B\left(0, t_{1}\right): h(x)=\tau, \nabla h(x) \neq 0\right\}} \sum_{i, j=1}^{n}|\nabla h|^{-1} \mathfrak{b}_{i j}(x) h_{x_{i}} h_{x_{j}} d \mathcal{H}^{n-1}=\breve{a}
$$

where $\breve{a}$ is a constant independent of $\tau \in\left[m\left(t_{2}, h\right), \gamma\right]$. To prove this claim, let $\tau_{1}, \tau_{2}, \epsilon$ be so that $m\left(t_{2}, h\right)<\tau_{1}<\tau_{2}<\gamma$ and

$$
0<\epsilon<\frac{1}{8} \min \left\{\tau_{1}-m\left(t_{2}, h\right), \gamma-\tau_{2}, \tau_{2}-\tau_{1}\right\}
$$

Let $l$ be infinitely differentiable on $\mathbb{R}$ with $l \equiv 1$ on $\left[\tau_{1}, \tau_{2}\right], l \equiv 0$ on $\mathbb{R} \backslash\left[\tau_{1}-\epsilon, \tau_{2}+\epsilon\right]$, and $|\nabla l| \leq c / \epsilon$ where $c$ is an absolute constant. Using $l \circ h$ as a test function in the weak formulation of (4.13) and using (4.14) and (4.15) we see that

$$
0=\int_{\mathbb{R}^{n}} \sum_{i, j=1}^{n} \mathfrak{b}_{i j}(x) h_{x_{i}} h_{x_{j}}\left(l^{\prime} \circ h\right) d y=\int_{\tau_{1}-\epsilon}^{\tau_{1}} I(s) l^{\prime}(s) d s+\int_{\tau_{2}}^{\tau_{2}+\epsilon} I(s) l^{\prime}(s) d s .
$$

where we have used the version of the coarea theorem in [MSZ] which is permissible since $h \in W_{\text {loc }}^{1,2}\left(\mathbb{R}^{n} \backslash \bar{B}(0,1)\right)$. From Lemma 4.2 applied to $V$, (4.14), (4.15), and 
once again the coarea theorem, we deduce that $I$ is integrable on any compact subset of $\left(m\left(t_{2}, h\right), \gamma\right)$. This observation, (4.21), and the Lebesgue differentiation theorem imply

$$
I\left(\tau_{2}\right)-I\left(\tau_{1}\right)=\int_{\tau_{1}-\epsilon}^{\tau_{1}}\left[I(s)-I\left(\tau_{1}\right)\right] l^{\prime}(s) d s+\int_{\tau_{2}}^{\tau_{2}+\epsilon}\left[I(s)-I\left(\tau_{2}\right)\right] l^{\prime}(s) d s \rightarrow 0 \quad \text { as } \epsilon \rightarrow 0
$$

for $\mathcal{H}^{1}$-almost every $\tau_{1}, \tau_{2} \in\left[m\left(t_{2}, h\right), \gamma\right]$. This proves claim (4.20).

Assuming $\breve{a} \neq 0$ and that $t_{0}>t_{2}$ we let

$$
\tilde{h}(x):= \begin{cases}\max \left(h(x)-m\left(t_{0}, h\right), 0\right) & \text { whenever } x \in \mathbb{R}^{n} \backslash \bar{B}\left(0, t_{1}\right), \\ 0 & \text { whenever } x \in \bar{B}\left(0, t_{1}\right) .\end{cases}
$$

Then from (4.19) we observe that

$$
\lim _{x \rightarrow y} \tilde{h}(x)=0 \quad \text { whenever } \quad y \in \partial B\left(0, t_{1}\right) .
$$

Let $0 \leq \phi \in C_{0}^{\infty}\left(B\left(0,4 t_{0}\right)\right)$ with $\phi \equiv 1$ on $\bar{B}\left(0,2 t_{0}\right)$ and $|\nabla \phi| \leq c t_{0}^{-1}$ where $c=c(n)$. Then from the above observation, (4.19) and Lemma 4.2 we see that $\tilde{h} \phi^{2}$ can be used as a test function in the weak formulation of (4.13). Doing this and using Hölder's inequality we get the Caccioppoli inequality,

$$
I_{1}:=\int \sum_{i, j=1}^{n} \mathfrak{b}_{i j}(x) \tilde{h}_{x_{i}} \tilde{h}_{x_{j}} \phi^{2} d x \leq c \int|\nabla V|^{n-2} \tilde{h}^{2}|\nabla \phi|^{2} d x=: I_{2} .
$$

Using (4.23), the coarea theorem from [MSZ], and (4.20) once again we arrive at

$$
\begin{aligned}
I_{1} & \geq \int_{\left\{x: 0<\tilde{h}(x)<m\left(2 t_{0}, h\right)-m\left(t_{0}, h\right)\right\}} \sum_{i, j=1}^{n} \mathfrak{b}_{i j}(x) \tilde{h}_{x_{i}} \tilde{h}_{x_{j}} \phi^{2} d x \\
& =\int_{0}^{m\left(2 t_{0}, h\right)-m\left(t_{0}, h\right)}\left(\int_{\{\tilde{h}=t\}}|\nabla \tilde{h}|^{-1} \sum_{i, j=1}^{n} \mathfrak{b}_{i j}(x) \tilde{h}_{x_{i}} \tilde{h}_{x_{j}} d \mathcal{H}^{n-1}\right) d t \\
& =\breve{a}\left[m\left(2 t_{0}, h\right)-m\left(t_{0}, h\right)\right]
\end{aligned}
$$

Also from Lemma 4.2 (b) for $V$ and the definition of $\tilde{h}$ we find

$$
I_{2} \leq c\left[M\left(4 t_{0}, h\right)-m\left(t_{0}, h\right)\right]^{2} \leq c^{2}\left[m\left(2 t_{0}, h\right)-m\left(t_{0}, h\right)\right]^{2} \neq 0
$$

where to get the last two inequalities we have used (4.19) and Harnack's inequality for $h-m\left(t_{0}, h\right)$ in $\mathbb{R}^{n} \backslash \bar{B}\left(0, t_{0}\right)$. Using (4.24) and (4.25) we conclude for some $\tilde{c} \geq 1$ that

$$
\breve{a} \leq \tilde{c}\left[m\left(2 t_{0}, h\right)-m\left(t_{0}, h\right)\right] .
$$

We now repeat this argument with $t_{0}$ replaced by $2^{k} t_{0}$ for $k=1,2, \ldots$ in (4.26). Summing the resulting inequalities we eventually get a contradiction to (4.17) unless $\breve{a}=0$. If $\breve{a}=0$ we observe that

$$
B\left(0,4 t_{0}\right) \backslash \bar{B}\left(0, t_{0}\right) \subset\left\{x: m\left(t_{0}, h\right)<h(x)<M\left(4 t_{0}, h\right)\right\} .
$$


Using this observation, the coarea theorem from [MSZ], (4.20), and arguing as in (4.24) we get $I_{1}=0$. It then follows from (4.15) that $\nabla \tilde{h} \equiv 0$ in $B\left(0,2 t_{0}\right) \backslash \bar{B}\left(0, t_{0}\right)$ and thereupon from Harnack's inequality that $h \equiv \gamma$ in $\mathbb{R}^{n} \backslash B\left(0, t_{0}\right)$, a contradiction to (4.19). Since $t_{0}>t_{2}$ is arbitrary we conclude from all these contradictions that (4.18) holds when $M(\cdot, h)$ and $m(\cdot, h)$ are both non-decreasing on $\left[t_{1}, \infty\right)$.

If $M(\cdot, h)$ and $m(\cdot, h)$ are both non-increasing on $\left[t_{1}, \infty\right)$ then either $M(t, h)=\gamma$ for some $t \geq t_{1}$ in which case we put $t_{2}=t$ and observe that (4.18) holds or there exists $t_{2}>t_{1}$ so that

$$
\gamma<M(t, h)<m\left(t_{1}, h\right) \text { for } t \geq t_{2}
$$

In this case let $t_{0}>t_{2}$ and define

$$
\hat{h}(x)= \begin{cases}\max \left(M\left(t_{0}, h\right)-h(x), 0\right) & \text { whenever } x \in \mathbb{R}^{n} \backslash \bar{B}\left(0, t_{1}\right), \\ 0 & \text { whenever } x \in B\left(0, t_{1}\right) .\end{cases}
$$

Repeating the argument from (4.23)-(4.26) with $\tilde{h}$ replaced by $\hat{h}$ and using arbitrariness of $t_{0}$ we eventually arrive at a contradiction so (4.18) is valid. The only other possible case in view of (4.17) is that there exist $t_{1}>1$, such that $M(\cdot, h)$ is nonincreasing on $\left[t_{1}, \infty\right)$ and $m(\cdot, h)$ is non-decreasing on $\left[t_{1}, \infty\right)$. In this case we see that either (4.18) is valid for some $t_{2} \geq t_{1}$ or

$$
m\left(t_{0}, h\right)<\gamma<M\left(t_{0}, h\right) \text { for all } t_{0} \in\left[t_{1}, \infty\right) .
$$

In this case we can apply Harnack's inequality to $M\left(t_{0}, h\right)-h$ and $h-m\left(t_{0}, h\right)$ in $\mathbb{R}^{n} \backslash \bar{B}\left(0, t_{0}\right)$ whenever $t_{0}>t_{1}$ to get

$$
M\left(t_{0}, h\right)-m\left(2 t_{0}, h\right) \leq c_{1}\left[M\left(t_{0}, h\right)-M\left(2 t_{0}, h\right)\right]
$$

and

$$
M\left(2 t_{0}, h\right)-m\left(t_{0}, h\right) \leq c_{1}\left[m\left(2 t_{0}, h\right)-m\left(t_{0}, h\right)\right] .
$$

Adding these two inequalities and doing some arithmetic we obtain

$$
M\left(2 t_{0}, h\right)-m\left(2 t_{0}, h\right) \leq \kappa\left(M\left(t_{0}, h\right)-m\left(t_{0}, h\right)\right)
$$

where $\kappa=\frac{c_{1}-1}{c_{1}+1} \in(0,1)$. Iterating this inequality with $t_{0}$ replaced by $2^{k} t_{0}, k=1,2, \ldots$, it follows that if $s \geq 2 t_{0}$, then

$$
M(s, h)-m(s, h) \leq c\left(t_{0} / s\right)^{\tilde{\alpha}}\left(M\left(t_{0}, h\right)-m\left(t_{0}, h\right)\right) \leq c^{2}\left(t_{1} / s\right)^{\tilde{\alpha}}
$$

for some $c \geq 1$, depending only on the data.

Finally, we are in a position to prove (4.12) in Lemma 4.3. First if (4.18) is valid, then using $r^{-1} h(r \omega)=\frac{\partial V(r \omega)}{\partial r}$ when $|x|=r$ and $\omega=x /|x| \in \mathbb{S}^{n-1}$ we find upon integrating (4.18) from $r=R \geq t_{2}$ to $\tau>2 R$ that

$$
V(\tau \omega)-V(R \omega)=\gamma \log (\tau / R)
$$

Otherwise, (i.e. (4.28) holds and therefore (4.30) holds) we find from (4.30) that if $\omega \in \mathbb{S}^{n-1}$ then

$$
|h(s \omega)-\gamma| \leq c_{++}\left(t_{1} / s\right)^{\tilde{\alpha}} \quad \text { for } s \geq 2 R \geq 4 t_{1}
$$


where $c_{++}$depends only on the data. Integrating this inequality with respect to $s$ from $R$ to $\tau>R$ we get as in (4.31) that

$$
|V(\tau \omega)-V(R \omega)-\gamma \log (\tau / R)| \leq c_{+}\left(t_{1} / R\right)^{\tilde{\alpha}}
$$

whenever $\omega \in \mathbb{S}^{n-1}$ and $\tau \geq 2 R \geq 4 t_{1}$. If either (4.31) or (4.32) holds we let

$$
\psi_{m}(y)=V(m y /|y|)-\gamma \log m \quad \text { whenever } y \in \mathbb{R}^{n} \backslash\{0\} \quad \text { for } m=2,3, \ldots
$$

Note that $\psi_{m}$ is a homogeneous of degree zero function for $m=2,3, \ldots$. From Lemma $4.2(b)$ for $V$ in $\mathbb{R}^{n} \backslash\{0\}$ and Lemmas 3.3-3.5, we see that $\nabla \psi_{m} \in C^{0, \sigma}(B(0,4) \backslash$ $B(0,1 / 4))$ for some $\sigma \in(0,1)$ with norm $\leq c$, while from (4.31) or (4.32) we deduce for $m \geq 2 R$ that $\left\{\psi_{m}\right\}$ is uniformly bounded by a constant depending on $R$. Using Ascoli's theorem we conclude that a sub-sequence of $\left\{\psi_{m}\right\}$ say $\left\{\psi_{m_{l}}\right\}$ converges uniformly in the $C^{1, \sigma}(B(0,2) \backslash B(0,1 / 2))$ norm to $b$, a homogeneous degree 0 function on $\mathbb{R}^{n} \backslash\{0\}$. Letting $\tau=m_{l}, \omega=x /|x|$ in (4.31) or (4.32), and $l \rightarrow \infty$ we conclude (4.12) for a fixed $x$ with $|x|=R \geq R_{0}$ where $R_{0}=2 t_{2}$ if (4.31) holds and $R_{0}=2 t_{1}$ if (4.32) holds. Now letting $R$ vary we get Lemma 4.3.

Finally, we prove

Lemma 4.4. Let $\mathcal{A} \in M_{n}(\alpha)$. Then there exists a unique (up to an additive constant) fundamental solution $F$ to the equation $\nabla \cdot \mathcal{A}(\nabla F)=0$ in $\mathbb{R}^{n}$, with pole at 0 , in the sense of (4.1). Furthermore, there exist $b \in C^{1, \sigma}(B(0,2) \backslash B(0,1 / 2)), \gamma>0$, and $\tilde{c} \geq 1$ such that

$$
\begin{aligned}
& (+) \quad F(z)=\gamma \log |z|+b(z /|z|) \quad \text { whenever } z \in \mathbb{R}^{n} \backslash\{0\}, \\
& (++) \quad \tilde{c}^{-1} \leq\langle z, \nabla F(z)\rangle \leq|z| \nabla F(z) \mid \leq \tilde{c} \quad \text { whenever } z \in \mathbb{R}^{n} \backslash\{0\} .
\end{aligned}
$$

Proof. Let $x=m y,|y| \geq 1 / m$, for $m \geq 4 R_{0}^{2}$ where $R_{0}$ is as above, and put

$$
F_{m}(y)=V(x)-\gamma \log m \quad \text { for } y \in \mathbb{R}^{n} \backslash \bar{B}(0,1 / m) .
$$

Then from Lemma 4.3 with $R=|x|$ we get for $|y| \geq m^{-1 / 2}$ that

$$
\begin{aligned}
\left|F_{m}(y)-\gamma \log \right| y|-b(y /|y|)| & =|V(x)-\gamma \log | y|-\gamma \log m-b(y /|y|)| \\
& =|V(m y)-\gamma \log m| y \mid-b(m y /(m|y|) \mid \\
& \leq c\left(R_{0} / m|y|\right)^{\alpha} \leq c\left(R_{0}^{2} / m\right)^{\alpha / 2}
\end{aligned}
$$

Also from the chain rule and Lemma 4.3 for $V$ we have if $|y|>1 / m$ that

$$
c^{-1} \leq\left\langle\nabla F_{m}(y), y\right\rangle \leq|y|\left|\nabla F_{m}(y)\right| \leq c
$$

where $c \geq 1$ depends only on the data. From Lemma 4.2, (4.34), (4.35), as well as Lemmas 3.3-3.5, we observe that the $C^{1, \sigma}$ norms of $\left\{F_{m}\right\}_{\{m \geq 16\}}$ are uniformly bounded on a given compact subset of $\mathbb{R}^{n} \backslash\{0\}$ for $m$ large enough. From this observation and (4.34) we conclude that

$$
\lim _{m \rightarrow \infty} F_{m}(y)=\gamma \log |y|+b(y /|y|)=F(y) \quad \text { whenever } y \in \mathbb{R}^{n} \backslash\{0\} .
$$

Thus, (4.33) $(+)$ is proved. Also (4.33) $(++)$ follows from (4.35) and uniform convergence of $\nabla F_{m} \rightarrow \nabla F$ on compact subsets of $\mathbb{R}^{n} \backslash\{0\}$. Moreover, $F$ is $\mathcal{A}$-harmonic in 
$\mathbb{R}^{n} \backslash\{0\}$. Extend $F_{m}+\gamma \log m$ to a continuous function on $\mathbb{R}^{n}$ by putting this function $\equiv 0$ on $\bar{B}(0,1 / m)$. Let $\mu_{m}$ denote the measure as in Lemma $3.7(i)$ corresponding to $F_{m}+\gamma \log m$, with support contained in $\partial B(0,1 / m)$. Once again using invariance of $\mathcal{A}$-harmonic functions under dilation and translation we deduce that

$$
\mu_{m}(\bar{B}(0,1 / m))=\Theta(\bar{B}(0,1))=1 \text {. }
$$

Using uniform convergence of $\nabla F_{m} \rightarrow \nabla F$ on compact subsets of $\mathbb{R}^{n} \backslash\{0\}$ and (4.35) we conclude for $\theta \in C_{0}^{\infty}\left(\mathbb{R}^{n}\right)$ that

$$
\int\langle\mathcal{A}(\nabla F), \nabla \theta\rangle d x=\lim _{m \rightarrow \infty} \int\left\langle\mathcal{A}\left(\nabla F_{m}\right), \nabla \theta\right\rangle d x=-\lim _{m \rightarrow \infty} \int \theta d \mu_{m}=-\theta(0) .
$$

Thus (4.1) $(i)$ and $(i i)$ are valid so $F$ is a fundamental solution in the sense of Definition 4.1 .

To prove uniqueness of $F$ up to a constant, suppose $F_{1}$ also satisfies (4.1) $(i)$ and (ii). We assume, as we may, that $F_{1}\left(e_{1}\right)=1$ where $e_{1}=(1,0, \ldots, 0)$. Then

$$
A=\lim _{t \rightarrow 0} m\left(t, F_{1}\right) \quad \text { and } \quad B=\lim _{t \rightarrow \infty} m\left(t, F_{1}\right)
$$

in the extended sense thanks to the minimum principle for $\mathcal{A}$-harmonic functions. We note that if $A$ is finite then $F_{1} \rightarrow A$ as $x \rightarrow 0$ while if $B$ is finite then $F_{1} \rightarrow B$ as $x \rightarrow \infty$. This note is proved using Harnack's inequality as in (4.16). We first show that

$$
A=-\infty .
$$

Indeed, if $A \neq-\infty$, then from the above note and the maximum-minimum principles for $\mathcal{A}$-harmonic functions we see that $A=\lim _{t \rightarrow 0} M\left(t, F_{1}\right)$ and $B \neq A$. In this case if $A>B$, let $s, t$ satisfy $A>s>t>B$ and set

$$
\hat{\theta}(x)=\max \left(\min \left(F_{1}, s\right), t\right)-t .
$$

Approximating $\hat{\theta}$ by $C_{0}^{\infty}\left(\mathbb{R}^{n}\right)$ functions we see that $\hat{\theta}$ can be used as a test function in (4.1) $(i i)$. Doing this it follows that

$$
-\hat{\theta}(0)=-s+t=\int_{\left\{x: t<F_{1}(x)<s\right\}}\left\langle\mathcal{A}\left(\nabla F_{1}\right), \nabla F_{1}\right\rangle d x .
$$

which is a contradiction since the right-hand integral is always non-negative as we see from the structural assumptions on $\mathcal{A}$. If $A<B$ we suppose $A<s<t<B$ and use

$$
\hat{\theta}(x)=\min \left(\max \left(F_{1}, s\right), t\right)-t
$$

as a test function in (4.1) $($ ii $)$ in order to obtain (4.38). Since $A \neq-\infty$ we can let $s \rightarrow A$ and take $t \geq M\left(1, F_{1}\right)$ in order to conclude from (4.38) that $F_{1} \in W^{1, n}(B(0,1) \backslash$ $\{0\})$. Using this conclusion and the fact that a point has zero $n$-capacity one can now show that $F_{1}$ extends to a $\mathcal{A}$-harmonic function in $B(0,1)$ which easily leads to a contradiction. To see this contradiction, given $\theta \in C_{0}^{\infty}(B(0,1))$ with $\theta(0) \neq 0$ and $\epsilon>0$, we let $\theta_{\epsilon}=\eta_{\epsilon} \theta$ where

$$
\eta_{\epsilon}(x)=\frac{\max (\log (|x| / \epsilon), 0)}{\log (1 / \epsilon)} \quad \text { whenever } x \in B(0,1) .
$$


It is easily shown that

$$
0 \leq \eta_{\epsilon} \leq 1, \quad \int_{B(0,1)}\left|\nabla \eta_{\epsilon}\right|^{n} d x \rightarrow 0, \quad \text { and } \eta_{\epsilon} \rightarrow 1 \text { a.e in } B(0,1) \quad \text { as } \epsilon \rightarrow 0 .
$$

Using $\theta_{\epsilon}$ as a test function in (4.1) (ii) and letting $\epsilon \rightarrow 0$ it follows from (2.1), $F_{1} \in W^{1, n}(B(0,1) \backslash\{0\}),(4.40)$, Hölder's inequality, and (4.1) (ii) for $\theta$ that

$$
\begin{aligned}
0 & =\int_{B(0,1)}\left\langle\mathcal{A}\left(\nabla F_{1}\right), \nabla \theta_{\epsilon}\right\rangle d x \\
& =\int_{B(0,1)} \theta\left\langle\mathcal{A}\left(\nabla F_{1}\right), \nabla \eta_{\epsilon}\right\rangle d x+\int_{B(0,1)} \eta_{\epsilon}\left\langle\mathcal{A}\left(\nabla F_{1}\right), \nabla \theta\right\rangle d x \\
& \leq \int_{B(0,1)} \theta\left|\nabla F_{1}\right|^{n-1}\left|\nabla \eta_{\epsilon}\right| d x+\int_{B(0,1)} \eta_{\epsilon}\left\langle\mathcal{A}\left(\nabla F_{1}\right), \nabla \theta\right\rangle d x \\
& \leq c\left(\int_{B(0,1)}\left|\nabla F_{1}\right|^{n} d x\right)^{(n-1) / n}\left(\int_{B(0,1)}\left|\nabla \eta_{\epsilon}\right|^{n} d x\right)^{1 / n}+\int_{B(0,1)} \eta_{\epsilon}\left\langle\mathcal{A}\left(\nabla F_{1}\right), \nabla \theta\right\rangle d x \\
& \rightarrow 0+\int_{B(0,1)}\left\langle\mathcal{A}\left(\nabla F_{1}\right), \nabla \theta\right\rangle d x=-\theta(0) \neq 0 \quad \text { as } \epsilon \rightarrow 0 .
\end{aligned}
$$

From this contradiction in (4.41) we conclude that (4.37) holds. Next we use (4.37) and $(4.1)(i)$ to show that

$$
d=\lim _{t \rightarrow 0} M\left(t, F_{1}\right)=-\infty .
$$

The proof is by contradiction. If $d \neq \pm \infty$, then from Harnack's inequality we find that $d=\lim _{t \rightarrow 0} m\left(t, F_{1}\right)$, a contradiction to (4.37). If $d=\infty$ then from the maximumminimum principles for $\mathcal{A}$-harmonic functions we deduce the existence of $t_{1}>0$ with $M\left(\cdot, F_{1}\right)$ non-increasing and $m\left(\cdot, F_{1}\right)$ non-decreasing on $\left(0, t_{1}\right)$. So arguing as in $(4.30)$ we get for some $\tilde{\alpha} \in(0,1)$ and $0<2 t<s<t_{1}$ that

$$
M\left(s, F_{1}\right)-m\left(s, F_{1}\right) \leq c(t / s)^{\tilde{\alpha}}\left(M\left(t, F_{1}\right)-m\left(t, F_{1}\right)\right) .
$$

Fix $s<t_{1}$ so that $m\left(s, F_{1}\right)<0$ and $M\left(s, F_{1}\right)>0$, and choose $x$ so that $|x|=t$ and $F_{1}(x)=\max \left(M\left(t, F_{1}\right),-m\left(t, F_{1}\right)\right)$. Then from (3.1) we have for some $\bar{c} \geq 1$,

$$
\left|F_{1}(x)\right|^{n} \leq \bar{c} t^{-n} \int_{B(x, t / 8)}\left|F_{1}(y)\right|^{n} d y .
$$

From (4.43), (4.44), and Hölder's inequality it follows that if $\lambda=2 / \tilde{\alpha}$, then

$$
\left[M\left(s, F_{1}\right)-m\left(s, F_{1}\right)\right]^{n \lambda}(s / t)^{2 n} \leq\left(\hat{c}\left|F_{1}(x)\right|\right)^{n \lambda} \leq \hat{c}^{2 \lambda n} t^{-n} \int_{B(x, t / 8)}\left|F_{1}(y)\right|^{n \lambda} d y
$$

where $\hat{c} \geq 1$ depends only on the data. Multiplying both sides of (4.45) by $t^{n}$ it follows that

$$
\int_{B(x, t / 8)}\left|F_{1}(y)\right|^{n \lambda} d y \rightarrow \infty \quad \text { as } t \rightarrow 0
$$


We have reached a contradiction since by $(4.1)(i)$ and Sobolev's inequality we have $\left|F_{1}\right|^{n \lambda}$ integrable on $B(0,1)$. Thus $(4.42)$ is valid. We put $F_{1}(0)=-\infty$ and observe from (4.42) that $F_{1}$ is continuous in the extended sense on $\mathbb{R}^{n}$.

The next step in our proof of Lemma 4.4 is to show using (4.42) that for $0<t<\infty$,

(a) $\left\{x: F_{1}(x)<m\left(t, F_{1}\right)\right\}$ is open, bounded, connected, and contains 0 ,

(b) $m\left(t, F_{1}\right)$ and $M\left(t, F_{1}\right)$ are strictly increasing on $(0, \infty)$.

To prove (4.46) (b) observe from (4.42) and the maximum principle for $\mathcal{A}$-harmonic functions that $M\left(\cdot, F_{1}\right)$ is at least non-decreasing on $(0, \infty)$. Also if $M\left(\cdot, F_{1}\right)$ is not strictly increasing then from Harnack's inequality for $\mathcal{A}$-harmonic functions it follows that $F_{1} \equiv$ constant in $B\left(0, t_{2}\right) \backslash \bar{B}\left(0, t_{1}\right)$ for some $0<t_{1}<t_{2}<\infty$. To get a contradiction choose $\theta \in C_{0}^{\infty}\left(B\left(0, t_{2}\right)\right)$ with $\theta \equiv 1$ in $\bar{B}\left(0, t_{1}\right)$. Using $\theta$ as a test function in (4.1) $(i i)$ we obtain a contradiction, since then the integral in this display is zero.

To prove $(4.46)(b)$ for $m\left(\cdot, F_{1}\right)$ by way of contradiction, observe by the same reasoning as above, that if $(b)$ is false for this function, then there exists $t_{1} \in(0, \infty)$ with $m\left(\cdot, F_{1}\right)$ strictly increasing on $\left(0, t_{1}\right)$ and strictly decreasing on $\left[t_{1}, \infty\right)$. In this case we can apply the same argument as in (4.43) to find for some $\tilde{\alpha} \in(0, \infty)$ that

$$
M\left(s, F_{1}\right)-m\left(s, F_{1}\right) \geq c^{-1}\left(s / t_{1}\right)^{\tilde{\alpha}}\left(M\left(t_{1}, F_{1}\right)-m\left(t_{1}, F_{1}\right)\right) .
$$

whenever $s>t_{1}$. Letting $s \rightarrow \infty$ in (4.47) we obtain a contradiction to our assumption that $\left|F_{1}(x)\right|=O(\log |x|)$ in a neighborhood of $\infty$.

From (4.46) (b) and the discussion above (4.37) it follows that

$$
\lim _{t \rightarrow \infty} M\left(t, F_{1}\right)=\lim _{t \rightarrow \infty} m\left(t, F_{1}\right)
$$

in possibly the extended sense. (4.46) (a) follows from this display, continuity of $F_{1}$ in the extended sense in $\mathbb{R}^{n}$, and the maximum principle for $\mathcal{A}$-harmonic functions.

Using (4.46) we show for given $r>0$ that $k=k(\cdot, r)=\max \left(F_{1}-m\left(r, F_{1}\right), 0\right)$ is an $\mathcal{A}$-subsolution on $\mathbb{R}^{n}$ with corresponding measure $\mu=\mu(\cdot, r)$ satisfying

$$
\operatorname{supp}(\mu) \subset\left\{x: F_{1}(x)=m\left(r, F_{1}\right)\right\} \quad \text { and } \quad \mu\left(\left\{x: F_{1}(x)=m\left(r, F_{1}\right)\right\}\right)=1 .
$$

To prove (4.48) we first note from (4.46) that $k \in W_{\text {loc }}^{1, n}\left(\mathbb{R}^{n}\right)$. Let $\psi=\left(k+\epsilon_{1}\right)^{\epsilon_{2}}-\epsilon_{1}^{\epsilon_{2}}$ where $\epsilon_{1}, \epsilon_{2}>0$ and suppose $0 \leq \theta \in C_{0}^{\infty}\left(\mathbb{R}^{n}\right)$. Using $\psi \theta$ as a test function in (4.1) (ii) for $\epsilon_{1}>0$ small, we obtain

$$
\begin{aligned}
0 & =\int\left\langle\mathcal{A}\left(\nabla F_{1}\right), \nabla k\right\rangle \epsilon_{2}\left(k+\epsilon_{1}\right)^{\left(\epsilon_{2}-1\right)} \theta d x+\int\left\langle\mathcal{A}\left(\nabla F_{1}\right), \nabla \theta\right\rangle \psi d x \\
& \geq \int\langle\mathcal{A}(\nabla k), \nabla \theta\rangle \psi d x .
\end{aligned}
$$

To get (4.49) we have used

$$
\int_{\left\{x: F_{1}(x)-m\left(r, F_{1}\right)>0\right\}}\left\langle\mathcal{A}\left(\nabla F_{1}\right), \nabla F_{1}\right\rangle \epsilon_{2}\left(k+\epsilon_{1}\right)^{\left(\epsilon_{2}-1\right)} \theta d x>0
$$


and also that

$$
\begin{aligned}
0 & \geq \int\left\langle\mathcal{A}\left(\nabla F_{1}\right), \nabla \theta\right\rangle \psi d x \\
& =\int_{\left\{x: F_{1}(x)-m\left(r, F_{1}\right)>0\right\}}\langle\mathcal{A}(\nabla k), \nabla \theta\rangle \psi d x \\
& =\int\langle\mathcal{A}(\nabla k), \nabla \theta\rangle \psi d x .
\end{aligned}
$$

Letting $\epsilon_{1} \rightarrow 0$ first and then $\epsilon_{2} \rightarrow 0$ in (4.49), we conclude from the Lebesgue dominated converge theorem and arbitrariness of $\theta$ that $k$ is an $\mathcal{A}$-subsolution on $\mathbb{R}^{n}$, so there exists $\mu$ as defined above (4.48). Let $K=\left\{x: F_{1}(x) \leq m\left(r, F_{1}\right)\right\}$. Then from (4.46) we see that $K$ is compact so there exists $0 \leq \theta \in C_{0}^{\infty}\left(\mathbb{R}^{n}\right)$ with $\theta \equiv 1$ on an open set containing $K$. Then from (4.1) (ii) and the integral inequality involving $k, \mu$ we obtain that

$$
\begin{aligned}
\mu(K)=\mu\left(\mathbb{R}^{n}\right) & =\int \theta d \mu=-\int\langle\mathcal{A}(\nabla k), \nabla \theta\rangle d x \\
& =-\int\left\langle\mathcal{A}\left(\nabla F_{1}\right), \nabla \theta\right\rangle d x=\theta(0)=1 .
\end{aligned}
$$

Also $\mu\left(\left\{x: F_{1}(x)<m\left(r, F_{1}\right)\right\}\right)=0$ since this set is open, bounded, and $\nabla k=0$ on it. Using arbitrariness of $\theta$ and regularity of $\mu$ we see that (4.48) is valid.

Next from (4.46) $(a)$ and facts about $n$-capacity we deduce that $\left\{x: F_{1}(x) \leq\right.$ $\left.m\left(r, F_{1}\right)\right\}$ is uniformly $(4 r, n)$-fat. Using this fact and (4.48) we see as in (4.2) $(a)$ that

$$
M(4 r, k)=M\left(4 r, F_{1}\right)-m\left(r, F_{1}\right) \approx m\left(2 r, F_{1}\right)-m\left(r, F_{1}\right) \approx 1
$$

whenever $0<r<\infty$. From (4.51) and Lemmas 3.3-3.5, it follows that

$$
M\left(2 r,\left|\nabla F_{1}\right|\right) \leq c r^{-1} \text {. }
$$

Now (4.51), (4.52), and $F_{1}\left(e_{1}\right)=1$ also imply that

$$
-F_{1}(x) \approx-\log |x| \text { whenever }|x| \leq 1 / 2 \quad \text { and } \quad F_{1}(x) \approx \log |x| \text { whenever }|x| \geq 2
$$

where all constants in (4.51)-(4.53) depend only on the data. Indeed, if $|x| \geq 2$, choose $k$ so that $2^{k} \leq|x| \leq 2^{k+1}$. Putting $r=2^{l}$ in (4.51) and summing the resulting inequality from 0 to $k$ we obtain (4.53) after simple estimates. A similar argument gives (4.53) if $|x| \leq 1 / 2$. To get a better inequality we note that if $F_{2}=\zeta F, \zeta$ a real number, then

$$
\hat{L}\left(F_{1}-F_{2}\right)=\sum_{i, j=1}^{n} \frac{\partial}{\partial x_{i}}\left(\hat{\mathfrak{b}}_{i j}(x)\left(F_{1}-F_{2}\right)_{x_{j}}\right)=0
$$

weakly in $\mathbb{R}^{n} \backslash\{0\}$ where

$$
\hat{\mathfrak{b}}_{i j}(x)=\int_{0}^{1} \frac{\partial \mathcal{A}_{i}\left(t \nabla F_{1}(x)+(1-t) \nabla F_{2}(x)\right)}{\partial \eta_{j}} d t
$$


for almost every $x \in \mathbb{R}^{n} \backslash\{0\}$. From (4.55) and the structural assumptions on $\mathcal{A}$ it follows that for fixed $x \in \mathbb{R}^{n} \backslash\{0\}$,

$$
\sum_{i, j=1}^{n} \hat{\mathfrak{b}}_{i j}(x) \xi_{i} \xi_{j} \approx\left(\left|\nabla F_{1}(x)\right|+\left|\nabla F_{2}(x)\right|\right)^{n-2}|\xi|^{2} \approx[(1+|\zeta|)|x|]^{2-n}|\xi|^{2}
$$

whenever $\xi \in \mathbb{R}^{n} \backslash\{0\}$. Here to get the last inequality we have used (4.52) and (4.33) $(++)$. Ratio constants depend only on the data. From (4.54)-(4.56) we see for $r \in(0, \infty)$ that $F_{1}-F_{2}$ is a bounded weak solution to a uniformly elliptic PDE with bounded measurable coefficients in $B(0,2 r) \backslash B(0, r / 2)$. Moreover, ellipticity constants and $L^{\infty}$ bound on the coefficients depend only on the data and $\zeta$. Put

$$
a_{1}=\liminf _{r \rightarrow \infty} m\left(r,\left(F_{1}-F\right) / F\right) .
$$

From (4.53) and our knowledge of $F$, we see that $F_{1} \approx F$ so that $-1<a_{1} \leq c$, where constants depend only on the data. Using this fact and Harnack's inequality it follows that

$$
a_{1}=\lim _{r \rightarrow \infty} M\left(r,\left(F_{1}-F\right) / F\right)=\lim _{r \rightarrow \infty} m\left(r,\left(F_{1}-F\right) / F\right) .
$$

To outline the proof of (4.57) observe from the definition of $a_{1}$ that there exists an increasing sequence $\left\{r_{j}\right\}$ with $r_{j} \rightarrow \infty$ as $j \rightarrow \infty$ such that $\left.m\left(r_{j}, F_{1}-F\right) / F\right) \rightarrow a_{1}$ as $j \rightarrow \infty$. Then for $j$ large enough, say $j \geq j_{0}$, and fixed $\epsilon>0$, small we have $F_{1}-F-\left(a_{1}-\epsilon\right) F \geq 0$ on $\partial B\left(0, r_{j}\right)$. Using the maximum principle for $\mathcal{A}$-harmonic functions in $B\left(0, r_{j}\right) \backslash B\left(0, r_{j_{0}}\right)$ and letting $j \rightarrow \infty$ it follows that $F_{1}-F-\left(a_{1}-\epsilon\right) F \geq 0$ on $\mathbb{R}^{n} \backslash B\left(0, r_{j_{0}}\right)$. Next we choose $j_{1}$ so that $r_{j_{1}} \geq 2 r_{j_{0}}$ and $F_{1}-F \leq\left(a_{1}+\epsilon\right) F$ at some point on $\partial B\left(0, r_{j}\right)$ for $j \geq j_{1}$. Using this choice and the previous facts, as well as (4.54)-(4.56) with $F_{2}=\left(1+a_{1}-\epsilon\right) F$, we see that Harnack's inequality can be applied on $\partial B\left(0, r_{j}\right)$ for $j \geq j_{1}$. Thus

$$
\max _{\partial B\left(0, r_{j}\right)}\left[F_{1}-F-\left(a_{1}-\epsilon\right) F\right] \leq c \min _{\partial B\left(0, r_{j}\right)}\left[F_{1}-F-\left(a_{1}-\epsilon\right) F\right] \leq c^{2} \epsilon \min _{\partial B\left(0, r_{j}\right)} F
$$

Using once again the maximum principle for $\mathcal{A}$-harmonic functions in $B\left(0, r_{j}\right) \backslash$ $B\left(0, r_{j_{1}}\right)$ and letting $j \rightarrow \infty$ we conclude for some $c^{\prime} \geq 0$, depending only on the data and $F_{1}\left(e_{1}\right)$ that

$$
F_{1}-F-a_{1} F \leq c^{\prime} \in F \quad \text { in } \mathbb{R}^{n} \backslash B\left(0, r_{j_{1}}\right) .
$$

Dividing both sides by $F$ and letting $\epsilon \rightarrow 0$ we get (4.57).

Next we put $F_{2}=\left(1+a_{1}\right) F$ and observe as in the display above (4.16) that

$$
a_{2}=\lim _{r \rightarrow 0} m\left(r, F_{1}-F_{2}\right) \quad \text { and } \quad a_{3}=\lim _{r \rightarrow 0} M\left(r, F_{1}-F_{2}\right) \quad \text { both exist. }
$$

if $\tilde{a} \in\left\{a_{3}, a_{2}\right\}$ is finite, then from (4.54)-(4.56) we see that Harnack's inequality can be used in a now well-known way to deduce $\tilde{a}=a_{3}=a_{2}$. Also, the case $a_{2}=-\infty, a_{3}=\infty$ cannot occur in view of (4.52)-(4.56), our knowledge of $F, F_{1}$, and the same argument as in (4.43). In order to rule out other cases choose $t_{1}>0$ so that $F(x) \geq 0$ in 
$\mathbb{R}^{n} \backslash B\left(0, t_{1}\right)$. Then given $\epsilon>0, t>t_{1}$, it follows from (4.33) and our choice of $a_{1}$ that there exists $s_{0}=s_{0}(\epsilon, t)>>t$ so that

$m\left(t, F_{1}-F_{2}\right)-\epsilon F \leq F_{1}-F_{2} \leq M\left(t, F_{1}-F_{2}\right)+\epsilon F$ on $\partial[B(0, s)-B(0, t)]$ for $s \geq s_{0}$.

Thus from the maximum - minimum principles for $\mathcal{A}$-harmonic functions, this inequality also holds in $B(0, s)-B(0, t)$. Letting $\epsilon \rightarrow 0$ we conclude that

$m\left(\cdot, F_{1}-F_{2}\right)$ is non-decreasing, and $M\left(\cdot, F_{1}-F_{2}\right)$ is non-increasing on $\left(t_{1}, \infty\right)$.

From the above discussion of cases we see that if $a_{2}=-\infty$, then $a_{3}=-\infty$. However from (4.60) and the maximum principle for $\mathcal{A}$-harmonic functions it then follows that $F_{1}-F_{2} \equiv$ constant, an obvious contradiction. If $a_{2}=\infty=a_{3}$ we can apply the same argument to get that $F_{1}-F_{2}$ has an absolute minimum so is constant, another contradiction. Also if $a_{2}=a_{3} \neq \pm \infty$, then (4.60) and the above maximum - minimum principles imply that

$$
m\left(\cdot, F_{1}-F_{2}\right) \geq a_{2} \geq M\left(\cdot, F_{1}-F_{2}\right) \text { on }(0, \infty)
$$

so $F_{1}-F_{2} \equiv$ constant. Finally $a_{1}=0$ since if $\theta \in C_{0}^{\infty}\left(\mathbb{R}^{n}\right)$ with $\theta(0)=1$, then from (4.1) (ii) for $F, F_{1}$, we have

$$
0=\int_{\mathbb{R}^{n}}\left\langle\mathcal{A}\left(\nabla F_{1}\right)-\mathcal{A}\left(\nabla F_{2}\right), \nabla \theta\right\rangle d x=\left(1+a_{1}\right)^{n-1}-1 .
$$

The proof of Lemma 4.4 is now complete.

\subsection{Existence and uniqueness for $p>n$.}

Definition 4.5. If $p>n \geq 2$ we say that $F$ is a fundamental solution to $\nabla \cdot \mathcal{A}(\nabla F)=$ 0 in $\mathbb{R}^{n}$ with pole at 0 if

(i) $F$ is $\mathcal{A}$-harmonic in $\mathbb{R}^{n} \backslash\{0\}$,

(ii) $F \in W_{\text {loc }}^{1, p}\left(\mathbb{R}^{n}\right), F$ is continuous in $\mathbb{R}^{n}, F(0)=0, F>0$ in $\mathbb{R}^{n} \backslash\{0\}$,

(iii) $\int\langle\mathcal{A}(\nabla F(z)), \nabla \theta(z)\rangle d z=-\theta(0) \quad$ whenever $\theta \in C_{0}^{\infty}\left(\mathbb{R}^{n}\right)$.

As in Lemma 4.4 we prove

Lemma 4.6. Let $2 \leq n$ be an integer and let $p$ be fixed with $n<p<\infty$. Let $\xi=(p-n) /(p-1)$ and assume that $\mathcal{A} \in M_{p}(\alpha)$ for some $\alpha \in(1, \infty)$. Then there exists a unique fundamental solution $F$ to the equation $\nabla \cdot \mathcal{A}(\nabla F)=0$ in $\mathbb{R}^{n}$ with pole at 0 in the sense of Definition 4.5. Furthermore, there exist $\sigma=\sigma(p, n, \alpha) \in(0,1)$, $c \geq 1$, depending only on the data, and $\psi \in C^{1, \sigma}\left(\mathbb{S}^{n-1}\right)$ such that

(i) $F(z)=|z|^{\xi} \psi(z /|z|)$ whenever $z \in \mathbb{R}^{n} \backslash\{0\}$,

(ii) $c^{-1} F(z)=\langle z, \nabla F(z)\rangle \leq|z||\nabla F(z)| \leq c F(z) \quad$ whenever $z \in \mathbb{R}^{n} \backslash\{0\}$.

Proof. A proof of Lemma 4.6 is given in Lemmas 5.1 and 5.2 of [LN4] and somewhat simpler than the case $p=n$ so we only sketch the details. Fix $R, 2<R<\infty$, and let $v=v(\cdot, R)$ be the $\mathcal{A}$-harmonic function in $B(0, R) \backslash\{0\}$ with continuous boundary 
value 0 at 0 and $R^{\xi}$ on $\partial B(0, R)$. Existence of $v \in W^{1, p}(B(0, R))$, follows from the fact that a point set has positive $\mathcal{A}$-capacity when $p>n$ (see [HKM, Chapter 2]). Extend $v$ to a continuous function in $B(0, R)$ by setting $v(0)=0$. Let $\nu$ denote the positive Borel measure with support at $\{0\}$ associated with $v$. As in Lemma 4.2 we prove

Lemma 4.7. Let $\nu, v, R$, and $\xi$ be as above. Then

(a) $\nu(\bar{B}(0, R)) \approx 1$

(b) $\quad c^{-1} v(x) \leq\langle\nabla v(x), x\rangle \leq|x||\nabla v| \leq c v(x) \quad$ whenever $x \in B(0, R) \backslash\{0\}$,

(c) $v(x) \approx|x|^{\xi} \quad$ whenever $x \in B(0, R) \backslash B(0,1)$

where ratio constants depend only on $p, n$, and $\alpha$.

Proof. To prove (4.63) let $w(x)=|x|^{\xi}$, when $x \in B(0, R)$, and note that $w$ is $p$ harmonic in $B(0, R) \backslash\{0\}$ with $v \equiv w$, continuously on $\partial B(0, R) \cup\{0\}$. Then as in (4.3) we also have

$$
\nu(\bar{B}(0,1)) R^{\xi}=\int_{B(0, R) \backslash \bar{B}(0,1)}\langle\mathcal{A}(\nabla v), \nabla v\rangle d x \approx \int_{B(0, R)}|\nabla w|^{\xi} d x \approx R^{\xi} .
$$

Thus $(a)$ in (4.63) is valid. To prove $(b)$ in (4.63), we show (compare with (4.6)) that if $1<\lambda \leq 101 / 100$ and $x \in B(0, R / \lambda) \backslash\{0\}$, then for some $c \geq 1$, depending only on the data

$$
c v(x) \leq \frac{v(\lambda x)-v(x)}{\lambda-1}
$$

Indeed this estimate is clearly valid at $x=0$. To show this inequality also holds when $x \in \partial B(0, R / \lambda)$ we first observe from Lemma 3.7 applied to $R^{\xi}-v$ and the same argument as in (4.5) that

$$
R^{\xi}-v(x) \approx R^{\xi} \quad \text { when }|x|=R / 8 .
$$

Let $\phi_{2}$ be as in (4.7). Then $\phi_{2} \equiv 1$ on $\partial B(0,1)$, $\equiv 0$ on $\partial B(0,8)$, and $\phi(8 x / R)$ is a subsolution at points in $B(0, R) \backslash B(0, R / 8)$ where $\nabla v \neq 0$, to the second order nondivergence form PDE for $v$ corresponding to $\mathcal{A}$-harmonicity when $N$ is sufficiently large (depending only on the data). Using these facts and (4.66) it follows as in (4.9) that

$$
R^{\xi}-v(x) \geq c^{-1} R^{\xi} \phi_{2}(8 x / R) \geq c^{-2} R^{\xi-1}(R-|x|)
$$

whenever $x \in B(0, R) \backslash B(0, R / 8)$. This inequality is easily seen to imply (4.65) whenever $x \in \partial B(0, R / \lambda), 1<\lambda \leq 101 / 100$. Letting $\lambda \rightarrow 1$ we get the left-hand inequality in (4.65). The far right inequality follows from (3.2) for $|x| \leq R / 2$ and from a barrier type argument using $R^{\xi}\left[1-\phi_{1}(8 x / R)\right], \phi_{1}$ as in (4.7), when $R / 2 \leq|x|<R$. Thus (4.65) is true. Letting $\lambda \rightarrow 1$ in this inequality we obtain the left-hand inequality in (4.63) (b). The right-hand inequality in this display follows from (3.2) ( $i i)$ for $x \in B(0, R / 2) \backslash B(0,2)$ and from the above barrier estimates if $1<|x|<2$ or $R / 2<|x|<R$. Finally, (4.63) (c) follows from uniform $(|x| / 2, p)$ fatness of $\{0\}$ and (3.5) $($ ii) . 
We now return to the proof of lemma 4.6. To avoid confusion we again temporarily write $v(\cdot, R), \nu(\cdot, R)$, for $v$ in Lemma 4.7. Using Ascoli's theorem and Lemmas 3.33.5 for $\mathcal{A}$-harmonic functions we see as $m \rightarrow \infty$ that a sub-sequence of $\{v(\cdot, m)\}_{m=8}^{\infty}$ converges uniformly on compact subsets of $\mathbb{R}^{n}$ to a $W_{\text {loc }}^{1, p}\left(\mathbb{R}^{n}\right)$ function $\hat{V} \geq 0$ which is $\mathcal{A}$-harmonic in $\mathbb{R}^{n} \backslash\{0\}$ with $\hat{V}(0)=0$. Let $\hat{\Theta}$ be the measure with support at $\{0\}$ corresponding to $\hat{V}$. We put

$$
F(\cdot):=\frac{\hat{V}(\cdot)}{[\hat{\Theta}(0))]^{1 /(p-1)}} .
$$

Then $(i)$ and $(i i)$ of (4.61) are true for $F$. (iii) of (4.61) follows from (3.5) (i) with $\tilde{u}=F$ since the point measure corresponding to $F$ has total mass 1 at $\{0\}$. Moreover (4.62) (ii) is true as we see from Lemma 4.7.

To prove uniqueness suppose that $F_{1}$ also satisfies $(4.61)(i)-(i i i)$. Then from (4.61) (iii) we see that the measure associated to $F_{1}$ has a point mass of total mass 1 at $\{0\}$ and since $\{0\}$ is uniformly $(R, p)$-fat for any $R>0$, it follows from (3.5) (ii) of Lemma 3.7 (as in (4.48)-(4.51)) that

$$
M\left(t, F_{1}\right)-m\left(t, F_{1}\right) \approx t^{\xi} \quad \text { whenever } t>0 .
$$

From (4.67) and (3.2) ( $\hat{a})$ we have

$$
M\left(t,\left|\nabla F_{1}\right|\right) \leq c t^{(1-n) /(p-1)} \quad \text { whenever } t>0 .
$$

Again constants depend only on the data. Using (4.67), (4.68), and (4.62) (ii) for $F$ we can now use the same argument as after (4.54) to deduce first that if $F_{2}=\zeta F$ then $F_{1}-F_{2}$ is a solution to an elliptic PDE as in (4.54), (4.55), whose ellipticity is given by (4.56) with $n$ replaced by $p$. Using these facts it then follows as in (4.57) that $\lim _{x \rightarrow \infty}\left(F_{1}-F\right) / F=a_{1}$, where $-1<a_{1}<\infty$. Next given $\epsilon>0$ small we find that $\left|F_{1}-\left(1+a_{1}\right) F\right| \leq \epsilon F$ on $\partial B(0, s)$ for $s>0$ large enough so from the maximum principle for $\mathcal{A}$-harmonic functions and $F(0)=0=F_{1}(0)$, this inequality also holds in $B(0, s)$. Letting $\epsilon \rightarrow 0$ we get $F_{1}=\left(1+a_{1}\right) F$. Now $a_{1}=0$ is proved in the same way as earlier (see the display before (4.61)). Thus $F$ is the unique function satisfying $(4.61)(i)-(i i i)$. Finally, since $\mathcal{A}$-harmonic functions are dilation invariant, it is easily checked that given $t>0$, the function $x \rightarrow t^{-\xi} F(t x)$, whenever $x \in \mathbb{R}^{n}$, also satisfies (4.61) $(i)-($ iii) so by uniqueness equals $F$. The proof of Lemma 4.6 is now complete.

\section{5. $\mathcal{A}$-harmonic Green's function}

Fix $p \geq n$, and let $F$ be the fundamental solution constructed in Lemma 4.4 when $p=n$ and in Lemma 4.6 when $n<p<\infty$. If $p=n$ we assume that $F\left(e_{1}\right)=1$. In this section we use $F$ to define, show the existence of, develop some properties of, and prove uniqueness for the $\mathcal{A}$-harmonic Green's function for $\mathbb{R}^{n} \backslash E$ with pole at $\infty$ when $p \geq n$ whenever $E$ is a certain compact convex set.

Definition 5.1 (A-harmonic Green's function). Given a compact, convex set $E \subset \mathbb{R}^{n}$ we say that $G$ is the $\mathcal{A}$-harmonic Green's function for $\mathbb{R}^{n} \backslash E$ with pole at $\infty$, 
if $G: \mathbb{R}^{n} \backslash E \rightarrow(0, \infty)$ has continuous boundary value 0 on $\partial E, G$ is $\mathcal{A}$-harmonic in $\mathbb{R}^{n} \backslash E$, and $G(x)=F(x)+k(x)$ where $k(x)$ is a bounded function in a neighborhood of $\infty$.

The statements and proof of uniqueness as well as some other properties of $G$ and $k$ are slightly different when $p>n$ and $p=n$, so we consider each range of $p$ separately. We start with the case when $p=n$.

Lemma 5.2. Let $p=n$. Given a compact convex set $E$ consisting of at least two points, there exists a unique $\mathcal{A}$-harmonic Green's function $G$ for $\mathbb{R}^{n} \backslash E$ with pole at $\infty$ in the sense of Definition 5.1. Moreover,

(a) $|\nabla G(x)| \leq c|x-\hat{x}|^{-1}$ whenever $x \in \mathbb{R}^{n} \backslash E$ and $\hat{x} \in E$ with $|x-\hat{x}| \geq 8 \operatorname{diam}(E)$ where $c$ depends only on the data.

(b) $\lim _{x \rightarrow \infty} k(x)=k(\infty)$ exists finitely.

(c) There exist $\beta \in(0,1)$, depending only on the data, and $\hat{r}_{0}=\hat{r}_{0}(E) \geq 100$ such that $|k(x)-k(\infty)| \leq \hat{r}_{0}|x|^{-\beta}$ whenever $|x| \geq \hat{r}_{0}$.

(d) If $\mu$ denotes the positive Borel measure associated to $G$ then $\mu(E)=1$.

Proof. To prove existence in Lemma 5.2 for $G$, let $E$ be a convex set consisting of at least two points and let $\hat{x}$ be as in Lemma 5.2. We first suppose $\hat{r}:=\operatorname{diam}(E)=1$ and $\hat{x}=0$. Then after proving Lemma 5.2 for these values, we shall use translation and dilation invariance of $\mathcal{A}$-harmonic functions to get this lemma for general $\hat{x}$ and $\hat{r}$. Let $R>4$ and $v=v(\cdot, R)$ be the $\mathcal{A}$-harmonic function in $B(0, R) \backslash E$ with $v(x) \equiv \gamma \log R$ on $\partial B(0, R)$ and $v \equiv 0$ on $\partial E$ in the sense of continuous boundary values. Here $\gamma$ is as in (4.33). Existence of $v$ and in fact local Hölder continuity of $v$ in $B(0, R)$ follows from the note after Definition 3.2. Extend $v$ to a continuous function in $B(0, R)$ by putting $v \equiv 0$ on $E$. Let $\nu$ be the measure corresponding to $v$ with support contained in $E$ as in Lemma 3.7 and let $\zeta(x)$ be the $n$-capacitary function for $E$ relative to $B(0, R)$. Since the $n$-capacity of a line segment of length 1 with center at the origin in $B(0, R)$ is $\approx(\log R)^{1-n}$, we see from Lemma 3.7, and the same argument as in (4.3) with $w(x)=(\log R)(1-\zeta(x))$, as well as (4.4) and (4.10) that

$$
1 \approx \nu(\bar{B}(0,1))^{1 /(n-1)} \approx M(2 t, v)-m(t, v) \approx M(2, v)
$$

whenever $2 \leq t \leq R / 2$. Using (5.2) and arguing as in (4.11) we get

$$
M(t,|\nabla v|) \leq c t^{-1} \quad \text { whenever } \quad 4 \leq t \leq R / 4 .
$$

Moreover, from (5.2), Harnack's inequality for $v$, the boundary maximum principle for $\mathcal{A}$-harmonic functions, and Lemma 4.4 we have

$$
|v(x)-F(x)| \leq c \quad \text { whenever } \quad 2 \leq|x| \leq R,
$$

where constants in (5.2)-(5.4) depend only on the data. From (5.2)-(5.4), Lemmas 3.3-3.5, and Ascoli's theorem we deduce that a subsequence, say $\left\{v\left(\cdot, R_{l}\right)\right\}$, converges uniformly on compact subsets of $\mathbb{R}^{n}$ as $R_{l} \rightarrow \infty$ to a Hölder continuous function, $G$, that is $\mathcal{A}$-harmonic in $\mathbb{R}^{n} \backslash E$ with $G \equiv 0$ on $E$. Moreover, $\left\{\nabla v\left(\cdot, R_{l}\right)\right\}$ converges 
uniformly on compact subsets of $\mathbb{R}^{n} \backslash E$ to $\nabla G$, and $\left\{\nu\left(\cdot, R_{l}\right)\right\}$ converges weakly as measures to $\mu$, the positive Borel measure corresponding to $G$. From this convergence, (5.2) and (5.3), we deduce that (5.1) $(a)$ is true when $\hat{x}=0$ and $\hat{r}=1$. Also (5.4) holds with $v$ replaced by $G$, so $G$ as in Definition 5.1 exists.

We next prove uniqueness. To this end, suppose $G_{1}$ is another $\mathcal{A}$-harmonic function in $\mathbb{R}^{n} \backslash E$ with continuous boundary value 0 on $\partial E$ and $G_{1}-F$ is bounded in a neighborhood of $\infty$. Then from the boundary maximum principle we see that $\mid G-$ $G_{1} \mid \leq a<\infty$ on $\mathbb{R}^{n} \backslash E$. From this inequality, the fact that $|G-F| \leq c$, and our knowledge of $F$, we deduce that for given $\epsilon>0$ there is an $s>0$ large such that $\left|G-G_{1}\right| \leq \epsilon G$ on $\partial B(0, s)$. Using the boundary maximum principle once again for $\mathcal{A}$-harmonic functions we conclude that this inequality also holds in $B(0, s) \backslash E$. Letting $\epsilon \rightarrow 0$ we obtain $G \equiv G_{1}$. Thus $G$ is unique.

It remains to prove $(b),(c)$ and $(d)$ of Lemma 5.2. To prove these inequalities, we note from (5.3) and Lemma 4.4 that $k=G-F$ satisfies the elliptic PDE in (4.54)(4.56) with $F_{1}=G, F_{2}=F$, and $\zeta=1$. Thus, $k$ is a solution to a uniformly elliptic PDE in divergence form in $B(0,2 r) \backslash \bar{B}(0, r / 2)$ when $r \geq 16$. Using this fact and boundedness of $k$ in $\mathbb{R}^{n} \backslash B(0,4)$ we can now essentially repeat the argument given for $h$ in the proof of Lemma 4.3. Thus, we first obtain as in (4.17) that

$$
\lim _{x \rightarrow \infty} k(x)=k(\infty)<\infty
$$

which proves (b) of Lemma 5.2. Then, using (5.5), we argue as in (4.18)-(4.27) to show that if $M(t, k)$ and $m(t, k)$ are both either ultimately non-decreasing or both ultimately non-increasing then $k \equiv$ a constant in a neighborhood of $\infty$. Thus in these cases, $(c)$ of Lemma 5.2 is trivially true. Otherwise, there exists $R_{0}$ with $M(\cdot, k)$ strictly decreasing and $m(\cdot, k)$ strictly increasing on $\left(R_{0}, \infty\right)$. Using this fact and Harnack's inequality we find as in (4.30) that $(c)$ of Lemma 5.1 is true in this case (when $\hat{x}=0$ and $\hat{r}=1$ ) with $\hat{r}_{0}=c R_{0}$.

To prove $(d)$ assuming $(c)$ when $\hat{x}=0$ and $\hat{r}=1$, let $R \geq 10 \hat{r}_{0} \geq 1000$ and $0 \leq \theta \in C_{0}^{\infty}(B(0,2 R))$ with $\theta \equiv 1$ on $B(0, R)$ and $|\nabla \theta| \leq c / R$. Using $\theta$ as a test function in (4.1) for $G$ we see that

$$
\mu(E)=-\int_{B(0,2 R) \backslash B(0, R)}\langle\mathcal{A}(\nabla G), \nabla \theta\rangle d x .
$$

Now from $C^{1}$ regularity of $\mathcal{A}$, the structural assumptions on $\mathcal{A}$ in Definition 2.1, Lemma 4.4, (5.1), we have

$$
|\mathcal{A}(\nabla F)-\mathcal{A}(\nabla G)||\nabla \theta| \leq c R^{1-n}|\nabla k| \quad \text { whenever } \quad R \leq|x| \leq 2 R
$$

where $c>0$ is a constant that depends only on the data. Also since $k$ is a solution to a uniformly elliptic PDE in $B(0,4 R) \backslash B(0, R / 2)$ it follows from a Caccioppoli type inequality for $k$ and $(c)$ of (5.1) that

$$
\int_{B(0,2 R) \backslash B(0, R)}|\nabla k| d x \leq c R^{n / 2}\left(\int_{B(0,2 R) \backslash B(0, R)}|\nabla k|^{2} d x\right)^{1 / 2} \leq c^{2} \hat{r}_{0} R^{n-1-\beta}
$$


From (5.6)-(5.8) and (4.1) (ii), we obtain

$$
\begin{aligned}
|\mu(E)-1| & =\left|\int_{B(0,2 R) \backslash B(0, R)}\langle\mathcal{A}(\nabla G)-\mathcal{A}(\nabla F), \nabla \theta\rangle d x\right| \\
& \leq c R^{1-n} \int_{B(0,2 R) \backslash B(0, R)}|\nabla k| d x \\
& \leq c^{3} \hat{r}_{0} R^{-\beta} \rightarrow 0 \text { as } R \rightarrow \infty .
\end{aligned}
$$

From (5.9) we conclude $(d)$ of (5.1) and so also Lemma 5.2 when $\hat{x}=0$ and $\hat{r}=1$. Now suppose that $\hat{x} \in E$ and $\operatorname{diam}(E)=\hat{r}$. Let $\hat{E}=(1 / \hat{r})(E-\hat{x})$. Then $0 \in \hat{E}$ and $\operatorname{diam}(\hat{E})=1$, so from our previous work, we see that $\hat{G}=F+\hat{k}$ where $\hat{G}$ is the $\mathcal{A}$-harmonic Green's function for $\mathbb{R}^{n} \backslash \hat{E}$ with pole at $\infty$. Let

$$
G(x)=\hat{G}\left(\frac{x-\hat{x}}{\hat{r}}\right) \quad \text { whenever } \quad x \in \mathbb{R}^{n} \backslash E .
$$

Using Lemma 5.2 for $\hat{E}$ and translation and dilation invariance of $\mathcal{A}$-harmonic functions we see that $G$ is $\mathcal{A}$-harmonic in $\mathbb{R}^{n} \backslash E$ with $G \equiv 0$ on $\partial E$. Also from Lemma 4.4 and Lemma 5.2 we can write for $x \in \mathbb{R}^{n} \backslash E$, that

$$
G(x)=\gamma \log \left(\frac{|x-\hat{x}|}{\hat{r}}\right)+b\left(\frac{x-\hat{x}}{|x-\hat{x}|}\right)+\hat{k}\left(\frac{x-\hat{x}}{\hat{r}}\right)=F(x)+k(x)
$$

where

$$
k(x)=-\gamma \log \hat{r}+\gamma \log \left(\frac{|x-\hat{x}|}{|x|}\right)+b\left(\frac{x-\hat{x}}{|x-\hat{x}|}\right)-b\left(\frac{x}{|x|}\right)+\hat{k}\left(\frac{x-\hat{x}}{\hat{r}}\right) .
$$

Using Lemma 5.1 for $\hat{k}$ and smoothness of $b$ we see first from (5.10) that

$$
k(\infty)=\lim _{x \rightarrow \infty} k(x)=\hat{k}(\infty)-\gamma \log (\hat{r})
$$

and second that there exists $\hat{r}_{0}=\hat{r}_{0}(E)$ with

$$
|k(x)-k(\infty)| \leq \hat{r}_{0}|x|^{-\beta} \quad \text { whenever } \quad|x| \geq \hat{r}_{0} .
$$

Also if $\hat{\mu}$ and $\mu$ denote the measures corresponding to $\hat{G}$ and $G$ respectively, then $\mu(E)=\mu(\hat{E})=1$, as follows easily from changing variables in the integral identity for $\hat{\mu}$. The proof of Lemma 5.2 is now complete.

Next we state

Lemma 5.3. Let $n<p<\infty$ be fixed and $\xi=(p-n) /(p-1)$. Given a nonempty convex compact set $E$, there exists a unique $\mathcal{A}$-harmonic Green's function $G$ for $\mathbb{R}^{n} \backslash E$ 
with pole at $\infty$ in the sense of Definition 5.1. Moreover

$(a)|\nabla G(x)| \leq c|x-\hat{x}|^{\xi-1} \approx \frac{G(x)}{|x-\hat{x}|}$ whenever $x \in \mathbb{R}^{n} \backslash E$ and $\hat{x} \in E$ with $|x-\hat{x}| \geq 8$ diam $(E)$ where $c$ depends only on the data.

(b) $\lim _{x \rightarrow \infty} k(x)=k(\infty)$ exists finitely and $k \leq 0$ in $\mathbb{R}^{n} \backslash E$.

(c) There exist $\beta \in(0,1)$, depending only on the data, and $\hat{r}_{0}=\hat{r}_{0}(E) \geq 100$ such that $|k(x)-k(\infty)| \leq \hat{r}_{0}|x|^{-\beta}$ whenever $|x| \geq \hat{r}_{0}$.

(d) If $\mu$ denotes the positive Borel measure associated to $G$ then $\mu(E)=1$.

Proof. Once again we first assume that $\hat{x}=0$ and $\hat{r}:=\operatorname{diam}(E)=1$. For fixed $p>n$, let $v=v(\cdot, R)$ be the $\mathcal{A}$-harmonic function in $B(0, R) \backslash E$ with boundary values $v \equiv 0$ on $E$ and $v \equiv R^{\xi}$ on $\partial B(0, R)$ in the continuous sense. Extend $v$ to a Hölder continuous function in $B(0, R)$ by setting $v \equiv 0$ on $E$. Let $\nu$ be the positive Borel measure associated to $v$ with support contained in $E$ as in Lemma 3.7. Using uniform $(R, p)$-fatness of $E$ and arguing as in (4.64), (4.67), we see that

$$
1 \approx \nu(B(0,2))^{1 /(p-1)} \approx t^{-\xi}[M(2 t, v)-m(t, v)] \approx M(2, v)
$$

whenever $4 \leq t \leq R / 4$. Using (5.13) and arguing as in (4.68) we get

$$
M(t,|\nabla v|) \leq c t^{(1-n) /(p-1)} \quad \text { whenever } \quad 8 \leq t \leq R / 8
$$

where constants in (5.13)-(5.14) depend only on the data. From (5.13)-(5.14), Lemmas 3.3-3.5, and Ascoli's theorem we deduce that a subsequence, say $\left\{v\left(\cdot, R_{l}\right)\right\}$, converges uniformly on compact subsets of $\mathbb{R}^{n}$ as $R_{l} \rightarrow \infty$ to a Hölder continuous function, $\bar{G}$ that is $\mathcal{A}$-harmonic in $\mathbb{R}^{n} \backslash E$ with $\bar{G} \equiv 0$ on $E$. Moreover, $\left\{\nabla v\left(\cdot, R_{l}\right)\right\}$ converges uniformly on compact subsets of $\mathbb{R}^{n} \backslash E$ to $\nabla \bar{G}$ and $\left\{\nu\left(\cdot, R_{l}\right)\right\}$ converges weakly as measures to $\bar{\mu}$, the positive Borel measure corresponding to $\bar{G}$. Let

$$
G(\cdot)=\frac{\bar{G}(\cdot)}{\bar{\mu}(B(0,2))^{1 /(p-1)}} \quad \text { and } \quad \mu(\cdot)=\frac{\bar{\mu}(\cdot)}{\bar{\mu}(B(0,2))} .
$$

Then $\mu$ is the measure corresponding to $G$ and $\mu(B(0,2))=1$.

Moreover from (5.13) and (5.14) we deduce that

$$
|\nabla G(x)| \leq c|x|^{(1-n) /(p-1)} \approx G(x) /|x| \approx F(x) /|x| \text { whenever } \quad|x| \geq 8 .
$$

Using these facts and the maximum principle for $\mathcal{A}$-harmonic functions we see that $F-G$ is a non-negative weak solution in $\mathbb{R}^{n} \backslash E$ to the elliptic equation in (4.54)-(4.55) with $F=F_{1}, G=F_{2}, \zeta=1$, and $n$ replaced by $p$. Also (4.56) is valid with $n$ replaced by $p$. Using this version of (4.54)-(4.56) we deduce as in (4.57) that

$$
\lim _{x \rightarrow \infty} \frac{(F-G)(x)}{G(x)}=a_{1}>-1 .
$$

Then from the maximum principle for $\mathcal{A}$-harmonic functions we see for given small $\epsilon>0$ 


$$
-\epsilon F-M(2, F) \leq\left(1+a_{1}\right) G-F \leq \epsilon F \quad \text { on } \partial(B(0, s) \backslash E)
$$

provided $s$ is large enough, so this inequality also holds in $B(0, s) \backslash E$. Letting $\epsilon \rightarrow 0$ we get

$$
0 \leq F-\left(1+a_{1}\right) G \leq M(2, F) \text { in } \mathbb{R}^{n} \backslash E .
$$

Using (5.17) and the new version of (4.54)-(4.56) we can now once again essentially repeat the argument given in the proof of Lemma 4.3 with $h=F-\left(1+a_{1}\right) G$. Thus if $k=\left(1+a_{1}\right) G-F$ we first obtain (5.5). Next using (5.5) we argue as in (4.17)-(4.26) to eventually conclude that $(c)$ of Lemma 5.3 is true for this $k$. It remains to show that $a_{1}=0$ in order to prove $(b),(c)$, of Lemma 5.3. To do this let $R>16,0 \leq \theta \in C_{0}^{\infty}(B(0,2 R))$ with $\theta \equiv 1$ on $B(0, R)$ and $|\nabla \theta| \leq c / R$. Using $\theta$ as a test function in (4.1) for $G$ we see that

$$
\left(1+a_{1}\right)^{p-1} \mu(E)=\left(1+a_{1}\right)^{p-1}=-\int_{B(0,2 R) \backslash B(0, R)}\left\langle\mathcal{A}\left(\left(1+a_{1}\right) \nabla G\right), \nabla \theta\right\rangle d x .
$$

Then as in (5.7) we get from (5.15), (4.62) (ii), and the structure assumptions on $\mathcal{A}$ for $k$ as defined above,

$\left|\mathcal{A}(\nabla F)-\mathcal{A}\left(\left(1+a_{1}\right) \nabla G\right)\right||\nabla \theta| \leq c\left(1+\left|a_{1}\right|\right)^{p-2} R^{\frac{n(2-p)-1}{p-1}}|\nabla k| \quad$ whenever $R \leq|x| \leq 2 R$.

Also (5.8) remains valid for our $k$. Using these inequalities we obtain as in (5.9)

$$
\begin{aligned}
\left|\left(1+a_{1}\right)^{p-1}-1\right| & =\left|\int_{B(0,2 R) \backslash B(0, R)}\left\langle\mathcal{A}\left(\left(1+a_{1}\right) \nabla G\right)-\mathcal{A}(\nabla F), \nabla \theta\right\rangle d x\right| \\
& \leq c\left(1+\left|a_{1}\right|\right)^{p-2} R^{\frac{n(2-p)-1}{p-1}} \int_{B(0,2 R) \backslash B(0, R)}|\nabla k| d x \\
& \leq c^{2}\left(1+\left|a_{1}\right|\right)^{p-2} \hat{r}_{0} R^{\frac{(n-1)-(p-1)(1+\beta)}{p-1}} \rightarrow 0 \quad \text { as } R \rightarrow \infty .
\end{aligned}
$$

From (5.20) we conclude $a_{1}=0$. Thus $(b)$ and $(c)$ of Lemma 5.3 are true when $\hat{r}=1$ and $\hat{x}=0$.

To prove uniqueness in this case suppose $G_{1}$ is also a $\mathcal{A}$-harmonic Green's function for $\mathbb{R}^{n} \backslash E$ with pole at $\infty$. Then from the boundary maximum principle for $\mathcal{A}$ harmonic functions we have $\left|G-G_{1}\right|$ bounded in $\mathbb{R}^{n} \backslash E$ so given $\epsilon>0$ it follows from Lemma $5.3(b)$ that if $s>0$ is large enough, then

$$
\left|G-G_{1}\right| \leq \epsilon G \quad \text { on } \partial(B(0, s) \backslash E) .
$$

Once again from the maximum principle this inequality holds in $B(0, s) \backslash E$. Letting $\epsilon \rightarrow 0$ we conclude $G_{1}=G$.

To remove the assumption that $0 \in E$ and $\operatorname{diam}(E)=1$, we suppose that $\hat{x} \in E$ and $\operatorname{diam}(E)=\hat{r}$ for some $\hat{x}$ and $\hat{r}>0$. Let $\hat{E}=(1 / \hat{r})(E-\hat{x})$. Then $0 \in \hat{E}$ and 
$\operatorname{diam}(\hat{E})=1$, so from our previous work, we see that $\hat{G}=F+\hat{k}$ where $\hat{G}$ is the $\mathcal{A}$-harmonic Green's function for $\mathbb{R}^{n} \backslash \hat{E}$ with pole at $\infty$ and $p>n$ fixed. Let

$$
G(x)=(\hat{r})^{\xi} \hat{G}\left(\frac{x-\hat{x}}{\hat{r}}\right) \quad \text { whenever } x \in \mathbb{R}^{n} \backslash E .
$$

Using Lemma 5.2 for $\hat{E}$ and translation and dilation invariance of $\mathcal{A}$-harmonic functions we see that $G$ is $\mathcal{A}$-harmonic in $\mathbb{R}^{n} \backslash E$ with $G \equiv 0$ on $\partial E$. Also from Lemma 4.4 and Lemma 5.2 we can write for $x \in \mathbb{R}^{n} \backslash E$, that

$$
G(x)=F(x-\hat{x})+\hat{r}^{\xi} \hat{k}\left(\frac{x-\hat{x}}{\hat{r}}\right)=F(x)+k(x)
$$

where

$$
k(x)=F(x-\hat{x})-F(x)+\hat{r}^{\xi} \hat{k}\left(\frac{x-\hat{x}}{\hat{r}}\right) .
$$

From this display and Lemma 5.3 in the previous special case we conclude first that

$$
\lim _{x \rightarrow \infty} k(x)=k(\infty)=\hat{r}^{\xi} \hat{k}(\infty)
$$

so $(b)$ of Lemma 5.3 is valid. Second we deduce the validity of $(c)$ of Lemma 5.3. Finally if $\hat{\mu}$ and $\mu$ denote the measures corresponding to $\hat{G}$ and $G$ respectively then $\mu(E)=\mu(\hat{E})=1$, as follows easily from changing variables in the integral identity involving $\hat{\mu}$. The proof of Lemma 5.3 is now complete.

We are now able to define $\mathcal{C}_{\mathcal{A}}(E)$ alluded to in the introduction.

Definition 5.4. If $E$ is a nonempty convex compact set when $p>n$ or $E$ contains at least two points when $p=n$ we put

$$
\mathcal{C}_{\mathcal{A}}(E):= \begin{cases}e^{-k(\infty) / \gamma} & \text { when } p=n \\ (-k(\infty))^{p-1} & \text { when } p>n\end{cases}
$$

Here $\gamma$ is the constant as in Lemma 4.4 .

Remark 5.5. From the definition of $\mathcal{C}_{\mathcal{A}}(E)$ and translation and dilation invariance of $\mathcal{A}$-harmonic functions we note that if $y \in \mathbb{R}^{n}, r>0$, and $E$ is a convex compact set containing at least two points with $\hat{E}=r(E+y)$, then

$$
\mathcal{C}_{\mathcal{A}}(\hat{E})= \begin{cases}r \mathcal{C}_{\mathcal{A}}(E) & \text { when } p=n \\ r^{p-n} \mathcal{C}_{\mathcal{A}}(E) & \text { when } p>n\end{cases}
$$

\section{Proof of Theorem A}

Let $\mathcal{A} \in M_{p}(\alpha)$ and $p \geq n$ be fixed. Let $G$ be the $\mathcal{A}$-harmonic Green's function for the complement of a nonempty compact convex set, $E$, containing at least two points when $p=n$ (see Lemma 5.2 for $p=n$ and Lemma 5.3 when $p>n$ ). In this section, we first study the levels of $G$ and then we prove that $\mathcal{C}_{\mathcal{A}}(\cdot)$ satisfies the Brunn-Minkowski inequality and also obtain the conclusion of Theorem A when equality occurs. We begin with 
Lemma 6.1. Let $p, \mathcal{A}, E$, and $G$ be as above and put $G \equiv 0$ in $E$. Then $\{x: G(x)<$ $t\}$ is convex for every $t \in(0, \infty)$. Moreover, $\nabla G \neq 0$ in $\mathbb{R}^{n} \backslash E$ and if $B\left(0, r_{0}\right) \subset E$ for some $r_{0}>0$ and $\operatorname{diam}(E)=1$ then

(a) $|\nabla G(x)| \approx\langle\nabla G, x /|x|\rangle \approx|x|^{(1-n) /(p-1)}$ wnenever $|x| \geq 4$,

(b) There exists $\tilde{\theta}>0$ and $c \geq 1$ depending only on $r_{0}$ and the data with

$$
|k(x)-k(\infty)| \leq c|x|^{-\tilde{\theta}} \text { whenever }|x| \geq 4 .
$$

Here proportional constants depend only on $r_{0}$ and the data.

Proof. Our proof of Lemma 6.1 is similar to the proof of Lemma 4.4 in [AGHLV]. Thus we shall often refer to this lemma for details. We first assume that

$$
\operatorname{diam}(E)=1,0 \in E, B\left(0, r_{0}\right) \subset E \text {, and }(2.6)(i) \text { holds for } \mathcal{A} \text {. }
$$

Given $R \geq 4$, let $v(\cdot)=v(\cdot, R)$ be the $\mathcal{A}$-harmonic function, defined as in the proof of Lemmas 5.2 and 5.3 , by

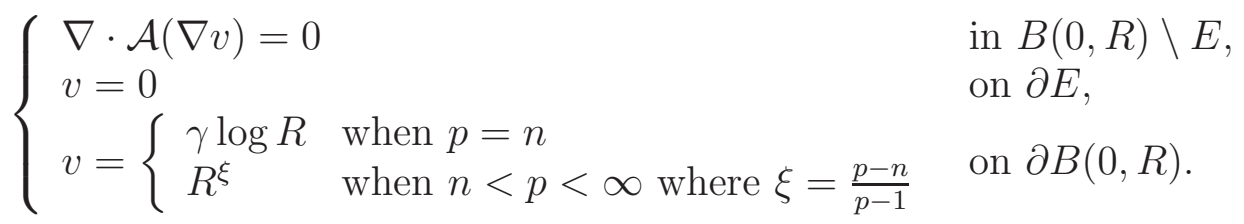

Here $\gamma$ is as in Definition 5.4. We claim there exists $c_{+} \geq 1$ such that if $x \in B(0, R) \backslash E$ then

$$
\begin{array}{lll}
\text { (a) } & c_{+}^{-1} \leq\langle x, \nabla v(x)\rangle & \text { when } p=n \\
\text { (b) } & c_{+}^{-1} v(x) \leq\langle x, \nabla v(x)\rangle & \text { when } p>n
\end{array}
$$

where $c_{+}$depends only on the data and $r_{0}$. To prove (6.4) we show for $1<\lambda \leq$ $101 / 100$ that if $x \in B(0, R / \lambda) \backslash E$ then

$$
\begin{aligned}
& \text { (a) } \frac{v(\lambda x)-v(x)}{\lambda-1} \geq c_{++}^{-1} \quad \text { when } p=n \text {, } \\
& \text { (b) } \frac{v(\lambda x)-v(x)}{\lambda-1} \geq c_{++}^{-1} v(x) \text { when } p>n
\end{aligned}
$$

where $c^{++}$depends only on the data and $r_{0}$. To prove (6.5) suppose $x, z \in \partial E$ with $|z-\lambda x|=d(\lambda x, E)$. Observe from convexity of $E$ and (6.2) that $d(\lambda x, E) \approx \lambda-1$, where constants depend only on $r_{0}$ and the data. Let $w=8 \frac{\lambda x-z}{|\lambda x-z|}$ and let $\phi_{1}$ and $\phi_{2}$ be as in (4.7). Then as in (4.8) we have for $N$ large enough that

$$
v(\lambda x) \geq c^{-1} v(w) \phi_{2}(\lambda x-w) \geq \tilde{c}^{-1}(\lambda-1),
$$

where $\tilde{c}$ has the same dependence as $c_{++}$. Thus (6.5) holds when $x \in \partial E$. The proof of (6.5) for $x \in \partial B(0, R / \lambda)$ is the same as in the right hand inequality in (4.9) and below (4.66). Using the boundary maximum principle for $\mathcal{A}$-harmonic functions we conclude the validity of (6.5) and letting $\lambda \rightarrow 1$ we obtain (6.4).

Extend $v$ to a Hölder continuous function in $\mathbb{R}^{n}$ by setting $v \equiv 0$ on $E$ while $v=\gamma \log R$ when $p=n$ and $v=R^{\xi}$ when $p>n$ on $\mathbb{R}^{n} \backslash B(0, R)$. Next we show that $\{x: v(x)<t\}$ is convex for every $t \in(0, \gamma \log R)$ when $p=n$ and $t \in\left(0, R^{\xi}\right)$ when 
$p>n$. In order to make easy reference to the proof of Lemma 4.4 in [AGHLV] we define $u(\cdot)=u(\cdot, R)$ by

$$
u(x):= \begin{cases}1-\frac{v(x)}{R^{\xi}} & \text { when } n<p<\infty, \\ 1-\frac{v(x)}{\gamma \log R} & \text { when } p=n .\end{cases}
$$

Then it can be easily seen that $0 \leq u \leq 1$ in $\mathbb{R}^{n}, u \equiv 1$ in $E, u \equiv 0$ on $\mathbb{R}^{n} \backslash B(0, R)$ continuously, and $u$ is $\tilde{\mathcal{A}}$-harmonic in $B(0, R) \backslash E$ where $\tilde{\mathcal{A}}(\eta)=-\mathcal{A}(-\eta)$ for $n \leq p<$ $\infty$. Note as in Remark 2.3 that $\tilde{\mathcal{A}}$ also satisfies the same structural properties as $\mathcal{A}$ (i.e., $\tilde{\mathcal{A}} \in M_{p}(\alpha)$ whenever $\mathcal{A} \in M_{p}(\alpha)$ ). From this observation, it can be easily seen that convexity of $\left\{x \in \mathbb{R}^{n} ; v(x)<t\right\}$ for $t$ in the range stated above is equivalent to convexity of $\left\{x \in \mathbb{R}^{n} ; u(x)>t\right\}$ for $0<t<1$. Moreover, it is shown in Lemma 4.4 of [AGHLV] that $\left\{x \in \mathbb{R}^{n}: u(x)>t\right\}$ is convex for every $t \in(0,1)$. We remark that the range of $p$ considered in [AGHLV] is $1<p<n$ and $u$ is the $\mathcal{A}$-capacitary function for $E$, so defined in $\mathbb{R}^{n} \backslash E$. However, the contradiction type argument in [AGHLV] uses only the maximum principle for $\mathcal{A}$-harmonic functions, assumption (6.2), and Lemmas 3.3-3.5 all of which hold in our situation. Thus $\{x: v(x)<t\}$ is convex for $t$ in the intervals stated above when (6.2) holds. Now we saw, in the proof of Lemmas 5.2 and 5.3, that a subsequence of $v(\cdot)=v(\cdot, R)$ converges uniformly on compact subsets of $\mathbb{R}^{n}$ to $G$ as $R \rightarrow \infty$ when $p=n$ and to $b G, b=$ constant when $p>n$. Moreover, the corresponding sequence of gradients converges uniformly on compact subsets of $\mathbb{R}^{n} \backslash E$ to $\nabla G$ for $p=n$ and to $b \nabla G$ when $p>n$. Thus $\{x: G(x)<t\}$ is convex for $t \in(0, \infty)$ and $(6.4)$ holds with $v$ replaced by $G$ whenever $x \in \mathbb{R}^{n} \backslash E$. To remove the assumption on $\mathcal{A}$ in (6.2) we approximate $\mathcal{A}$ by a sequence of smooth $\left\{\mathcal{A}^{(l)}\right\}$ where $\mathcal{A}^{(l)} \in M_{p}(\alpha)$ for $l=1,2, \ldots$ and take limits of the corresponding sequence $\left\{G^{(l)}\right\}$ (see the paragraph following (4.35) of Lemma 4.4 in [AGHLV] for more details). Thus (6.4) is valid for $\mathcal{A} \in M_{p}(\alpha)$ whenever $B\left(0, r_{0}\right) \subset E$ and $\operatorname{diam}(E)=1$. Now (6.4) and (5.1) (a) imply (6.1) (a) when $p=n$ while (6.4), (5.12) (a), (d), and (3.5) (ii) give (6.1) $(a)$ when $p>n$. To prove $(6.1)(b)$ observe that $m(t, k)$ is non-decreasing and $M(t, k)$ non-increasing on $(2, \infty)$ as follows from the maximum principle for $\mathcal{A}$ harmonic functions, boundedness of $k$, and our knowledge of $F$. Using this fact and arguing as in (4.30) we now get (6.1) (b).

Next if $E$ has nonempty interior, we can translate and dilate $E$ to get a convex set $\hat{E}$ with diameter 1 and $B\left(0, r_{0}\right) \subset \hat{E}$ for some $r_{0}>0$. Using (6.4) for the $\mathcal{A}$ harmonic Green's function for $\mathbb{R}^{n} \backslash \hat{E}$ with pole at infinity and then using a homothetic transformation to get back to $E$ we conclude from Remark 2.3 that Lemma 6.1 is valid whenever $E$ has nonempty interior.

If $E$ has empty interior choose $\hat{x} \in E$ and $\rho>0$ with $E \subset B(\hat{x}, \rho)$. Let $G^{(l)}$ denote the $\mathcal{A}$-harmonic Green's function for $\mathbb{R}^{n} \backslash\{x: d(x, E)<1 / l\}$ for $l=1,2, \cdots$ with pole at infinity and $\tilde{G}$ the $\mathcal{A}$-harmonic Green's function for $\mathbb{R}^{n} \backslash B(\hat{x}, \rho)$ with pole at infinity. Then for $l$ large enough it follows from the same argument used for proving uniqueness of $G$ that $\tilde{G} \leq G^{(l)} \leq G$. Using this fact, Lemmas 3.3-3.5, and Ascoli's theorem we find that a subsequence of $\left\{G^{(l)}\right\}$ converges uniformly to $G$ on compact 
subsets of $\mathbb{R}^{n}$ so $\{x: G(x)<t\}$ is convex for $t \in(0, \infty)$. Finally, we note that $G-t$ is the $\mathcal{A}$-harmonic Green's function for $\mathbb{R}^{n} \backslash\{x: G(x) \leq t\}$ with a pole at infinity. Since this set is convex with nonempty interior we have $\nabla G(x) \neq 0$ whenever $G(x)>t$ and $t>0$. The proof of Lemma 6.1 is now complete.

Remark 6.2. We note that if $\mathcal{A} \in M_{p}(\alpha)$ for fixed $\alpha \in(1, \infty)$ and $F$ is the fundamental solution with pole at $\{0\}$ when $p=n$ defined in Definition 4.1 then $\{x: F(x)<t\}$ is convex when $t \in(-\infty, \infty)$ as follows from our construction of $F$ and essentially the same proof we gave for $G$. Also observe that this remark is not needed when $p>n$ since the $\mathcal{A}$-harmonic Green's function $G$ with pole at infinity for $\mathbb{R}^{n} \backslash\{0\}$ and fundamental solution $F$ with pole at $\{0\}$ defined in Definition 4.5 are the same.

\subsection{Proof of the Brunn-Minkowski inequality.}

Proof of (2.4) and (2.5) in Theorem $A$. Let $E_{1}$ and $E_{2}$ be compact convex sets. Note from (5.23) that if $p>n$ and either $E_{1}$ or $E_{2}$ is a single point, then equality holds in (2.5) and Theorem $\mathrm{A}$ is trivially true. Thus we assume $E_{1}$ and $E_{2}$ each contain at least two points when $p \geq n$. For $i=1,2$, let $G_{i}$ be the $\mathcal{A}$-harmonic Green's function for $\mathbb{R}^{n} \backslash E_{i}$ with pole at $\infty$ obtained in Lemmas 5.2 and 5.3 for $n \leq p<\infty$. Let $\mathcal{C}_{\mathcal{A}}(\cdot)$ be defined as in Definition 5.4. For fixed $\lambda \in(0,1)$, let $G$ be the $\mathcal{A}$-harmonic Green's function for $\mathbb{R}^{n} \backslash\left[\lambda E_{1}+(1-\lambda) E_{2}\right]$ with pole at $\infty$. As observed in [B1, CS, AGHLV], it is enough to prove for arbitrary convex compact sets $E_{i}^{\prime}$ containing at least two points when $p \geq n$ for $i=1,2$ that

$$
\mathcal{C}_{\mathcal{A}}\left(E_{1}^{\prime}+E_{2}^{\prime}\right)^{\frac{1}{p-n}} \geq \mathcal{C}_{\mathcal{A}}\left(E_{1}^{\prime}\right)^{\frac{1}{p-n}}+\mathcal{C}_{\mathcal{A}}\left(E_{2}^{\prime}\right)^{\frac{1}{p-n}} \quad \text { when } n<p<\infty
$$

and

$$
\mathcal{C}_{\mathcal{A}}\left(E_{1}^{\prime}+E_{2}^{\prime}\right) \geq \mathcal{C}_{\mathcal{A}}\left(E_{1}^{\prime}\right)+\mathcal{C}_{\mathcal{A}}\left(E_{2}^{\prime}\right) \quad \text { when } p=n
$$

To get (2.5) from (6.8) when $n<p<\infty$ (and (2.4) from (6.9) when $p=n$ ) put

$$
E_{1}^{\prime}=\lambda E_{1} \quad \text { and } \quad E_{2}^{\prime}=(1-\lambda) E_{2}
$$

and use $(p-n)$-homogeneity of $\mathcal{C}_{\mathcal{A}}(\cdot)$ (and 1-homogeneity of $\mathcal{C}_{\mathcal{A}}(\cdot)$ when $p=n$ ). To get (6.8) from (2.5) (and to get (6.9) from (2.4)) one can take $\lambda=1 / 2$ and use $(p-n)$-homogeneity of $\mathcal{C}_{\mathcal{A}}(\cdot)$ when $n<p<\infty$ (and 1-homogeneity of $\mathcal{C}_{\mathcal{A}}(\cdot)$ when $p=n$ ) once again. On the other hand, to prove (6.8) when $n<p<\infty$ and (6.9) when $p=n$ it suffices to show, for all $\lambda \in(0,1)$, that

$$
\mathcal{C}_{\mathcal{A}}\left(\lambda E_{1}^{\prime \prime}+(1-\lambda) E_{2}^{\prime \prime}\right) \geq \min \left\{\mathcal{C}_{\mathcal{A}}\left(E_{1}^{\prime \prime}\right), \mathcal{C}_{\mathcal{A}}\left(E_{2}^{\prime \prime}\right)\right\}
$$

whenever $E_{i}^{\prime \prime}$ for $i=1,2$ are compact convex sets containing at least two points when $p \geq n$ and for fixed $p$ with $n \leq p<\infty$. To get (6.8) from (6.10) when $n<p<\infty$ let

$$
E_{i}^{\prime \prime}=\frac{E_{i}^{\prime}}{\mathcal{C}_{\mathcal{A}}\left(E_{i}^{\prime}\right)^{\frac{1}{p-n}}} \quad \text { for } i=1,2 \quad \text { and } \quad \lambda=\frac{\mathcal{C}_{\mathcal{A}}\left(E_{1}^{\prime}\right)^{\frac{1}{p-n}}}{\mathcal{C}_{\mathcal{A}}\left(E_{1}^{\prime}\right)^{\frac{1}{p-n}}+\mathcal{C}_{\mathcal{A}}\left(E_{2}^{\prime}\right)^{\frac{1}{p-n}}}
$$


then use $(p-n)$-homogeneity of $\mathcal{C}_{\mathcal{A}}(\cdot)$ and do some algebra. Similarly, to get $(6.9)$ from (6.10) when $p=n$ we let

$$
E_{i}^{\prime \prime}=\frac{E_{i}^{\prime}}{\mathcal{C}_{\mathcal{A}}\left(E_{i}^{\prime}\right)} \quad \text { for } i=1,2 \quad \text { and } \quad \lambda=\frac{\mathcal{C}_{\mathcal{A}}\left(E_{1}^{\prime}\right)}{\mathcal{C}_{\mathcal{A}}\left(E_{1}^{\prime}\right)+\mathcal{C}_{\mathcal{A}}\left(E_{2}^{\prime}\right)} .
$$

Finally, one can easily get (6.10) from (2.5) when $n<p<\infty$ (from (2.4) when $p=n$ ). Hence we conclude that (2.5), (6.8), and (6.10) are all equivalent when $n<p<\infty$. Similarly, (2.4), (6.9), and (6.10) are all equivalent when $p=n$. Therefore, we focus on proving (6.10) for $n \leq p<\infty$ and also for ease of notation we shall just use $E_{i}$ instead of $E_{i}^{\prime \prime}$ in (6.10). For fixed $\lambda \in(0,1)$, we claim that

$$
G(x) \leq G^{*}(x):=\inf \left\{\max \left\{G_{1}(y), G_{2}(z)\right\} ; \begin{array}{l}
x=\lambda y+(1-\lambda) z \\
\lambda \in[0,1], y, z \in \mathbb{R}^{n}
\end{array}\right\}
$$

whenever $x \in \mathbb{R}^{n}$. Assume that (6.11) holds for the moment and we show how to get (6.10) from (6.11). It follows from (6.11) and definition of the $\mathcal{A}$-harmonic Green's function that

$$
\begin{aligned}
-k(x)=F(x)-G(x) & \geq F(x)-G^{*}(x) \\
& \geq \min \left\{F(x)-G_{1}(x), F(x)-G_{2}(x)\right\}=\min \left\{-k_{1}(x),-k_{2}(x)\right\}
\end{aligned}
$$

where $F$ is as in Lemma 4.4 when $p=n$ and as in Lemma 4.6 when $n<p<\infty$. Here $k_{1}, k_{2}, k$ are the functions appearing in Lemma 5.2 when $p=n$ and in Lemma 5.3 when $n<p<\infty$ associated to $E_{1}, E_{2}, \lambda E_{1}+(1-\lambda) E_{2}$ respectively. Using the definition of $\mathcal{C}_{\mathcal{A}}(\cdot)$ and this inequality when $n<p<\infty$ we have

$$
\begin{aligned}
\mathcal{C}_{\mathcal{A}}\left(\lambda E_{1}+(1-\lambda) E_{2}\right) & =(-k(\infty))^{p-1}=\lim _{|x| \rightarrow \infty}[F(x)-G(x)]^{p-1} \\
& \geq \min \left\{\lim _{|x| \rightarrow \infty}\left[F(x)-G_{1}(x)\right]^{p-1}, \lim _{|x| \rightarrow \infty}\left[F(x)-G_{2}(x)\right]^{p-1}\right\} \\
& =\min \left\{\left(-k_{1}(\infty)\right)^{p-1},\left(-k_{2}(\infty)\right)^{p-1}\right\}=\min \left\{\mathcal{C}_{\mathcal{A}}\left(E_{1}\right), \mathcal{C}_{\mathcal{A}}\left(E_{2}\right)\right\} .
\end{aligned}
$$

While when $p=n$, the inequality above gives us (where $\gamma$ is as in Remark 5.5)

$$
\begin{aligned}
\mathcal{C}_{\mathcal{A}}\left(\lambda E_{1}+(1-\lambda) E_{2}\right) & =e^{-k(\infty) / \gamma}=\lim _{|x| \rightarrow \infty} e^{\frac{F(x)-G(x)}{\gamma}} \\
& \geq \min \left\{\lim _{|x| \rightarrow \infty} e^{\frac{F(x)-G_{1}(x)}{\gamma}}, \lim _{|x| \rightarrow \infty} e^{\frac{F(x)-G_{2}(x)}{\gamma}}\right\} \\
& =\min \left\{e^{-k_{1}(\infty) / \gamma}, e^{-k_{2}(\infty) / \gamma}\right\}=\min \left\{\mathcal{C}_{\mathcal{A}}\left(E_{1}\right), \mathcal{C}_{\mathcal{A}}\left(E_{2}\right)\right\} .
\end{aligned}
$$

Hence (6.10) is true for all $p$ with $n \leq p<\infty$ and in view of our earlier remarks we conclude that the proof of (2.5) when $n<p<\infty$ and (2.4) when $p=n$ in Theorem A are complete assuming (6.11).

We now return to the proof of (6.11). As in the proof of Lemma 6.1, for $R>>1$ we consider $\tilde{v}(\cdot)=\tilde{v}(\cdot, R)$ which is the solution to the Dirichlet problem as in $(6.3)$ where $v$ is replaced by $\tilde{v}$ whenever $\tilde{v} \in\left\{v_{1}, v_{2}, v\right\}$ corresponding to $\tilde{E} \in\left\{E_{1}, E_{2}, \lambda E_{1}+\right.$ $\left.\left.(1-\lambda) E_{2}\right)\right\}$. Extend $\tilde{v}$ to $\mathbb{R}^{n}$ by putting $\tilde{v} \equiv 0$ on $\tilde{E}$. Also if $p=n$, set $\tilde{v}=\gamma \log R$ and if $p>n$, set $\tilde{v}=R^{\xi}$ on $\mathbb{R}^{n} \backslash B(0, R)$. (where $\xi=(p-n) /(p-1)$ and $\gamma$ is as 
in Definition 5.4) when $n<p<\infty$. We know from the proof of Lemmas 5.2 and 5.3 that a subsequence of $\tilde{v}(\cdot)=\tilde{v}(\cdot, R) \in\left\{v_{1}, v_{2}, v\right\}$ converges uniformly on compact subsets of $\mathbb{R}^{n}$ to $\tilde{G} \in\left\{G_{1}, G_{2}, G\right\}$. From this observation, we see that in order to prove (6.11), it is enough to show that

$$
v(x) \leq v^{*}(x):=\inf \left\{\max \left\{v_{1}(y), v_{2}(z)\right\} ; \begin{array}{l}
x=\lambda y+(1-\lambda) z, \\
\lambda \in[0,1], y, z \in \mathbb{R}^{n}
\end{array}\right\}
$$

whenever $x \in \mathbb{R}^{n}$. Once again as in Lemma 6.1 , we consider $\tilde{u}(\cdot)=\tilde{u}(\cdot, R) \in$ $\left\{u_{1}, u_{2}, u\right\}$ which is defined as in (6.7) with $u$ is replaced by $\tilde{u}$ whenever $\tilde{v} \in\left\{v_{1}, v_{2}, v\right\}$. From this, we observe that $0 \leq \tilde{u} \leq 1$ in $\mathbb{R}^{n}, \tilde{u} \equiv 1$ in $\tilde{E}, \tilde{u} \equiv 0$ on $\mathbb{R}^{n} \backslash B(0, R)$ continuously, and $\tilde{u}$ is $\tilde{\mathcal{A}}$-harmonic in $B(0, R) \backslash \tilde{E}$ where $\tilde{\mathcal{A}}(\eta)=-\mathcal{A}(-\eta)$ for $n \leq p<\infty$. As observed earlier $\tilde{\mathcal{A}}$ also satisfies the same structural properties as $\mathcal{A}$. We see that (6.12) is equivalent to

$$
u(x) \geq u^{*}(x):=\sup \left\{\min \left\{u_{1}(y), u_{2}(z)\right\} ; \begin{array}{l}
x=\lambda y+(1-\lambda) z, \\
\lambda \in[0,1], y, z \in \mathbb{R}^{n}
\end{array}\right\}
$$

whenever $x \in \mathbb{R}^{n}$. A proof of (6.13) can be found in [AGHLV] at (5.13). Once again the range of $p$ in that article is for $1<p<n$ and $\mathcal{A}$-capacitary functions but uses only Lemmas 3.3-3.5 and the maximum principle for $\mathcal{A}$-harmonic functions so is valid in our situation. This finishes the proof of (6.13) and in view of our earlier observations proof of (2.5) when $n<p<\infty$ as well as (2.4) when $p=n$ in Theorem A.

6.2. Final proof of Theorem A. In this subsection our aim is to prove that if equality occurs in (2.5) when $n<p<\infty$ or in (2.4) when $p=n$ in Theorem $\mathrm{A}$ and if $\mathcal{A}$ satisfies the additional structural assumptions in (2.6) then $E_{2}$ is a translation and dilation of $E_{1}$. To do this, using the additional structural assumptions on $\mathcal{A}$, we first construct the corresponding fundamental solution explicitly.

6.2.1. Construction of the fundamental solution. In this subsection we begin with some observations when $\mathcal{A} \in M_{p}(\alpha)$ and additionally satisfies (2.6) (ii):

$$
\mathcal{A}_{i}=\frac{\partial f}{\partial \eta_{i}}(\eta), 1 \leq i \leq n, \text { where } f(t \eta)=t^{p} f(\eta) \text { when } t>0, \eta \in \mathbb{R}^{n} \backslash\{0\}
$$

so $f$ has continuous second partials on $\mathbb{R}^{n} \backslash\{0\}$. Using some ideas from [CS1], the fundamental solution was constructed in [AGHLV, section 7.2] when $1<p<n$ associated to $\mathcal{A}$-harmonic PDEs. In this part we extend this construction to include $n \leq p<\infty$.

We can write $f(\eta)=(k(\eta))^{p}$ and from (6.14) we see that $k(\eta)$ for $\eta \in \mathbb{R}^{n} \backslash\{0\}$ is homogeneous of degree 1 and has continuous second partials on $\mathbb{R}^{n} \backslash\{0\}$. Also in (7.4) of [AGHLV] it is shown that

$$
k^{2} \text { is strictly convex on } \mathbb{R}^{n} \text {. }
$$

From (6.15) and well-known properties of the support function for a convex set we see that if $X \in \mathbb{R}^{n} \backslash\{0\}$, then

$$
h(X)=\sup \{\langle\eta, X\rangle: \eta \in\{k \leq 1\}\}
$$


has continuous second partials and $h$ is homogeneous of degree 1. Moreover,

$$
\nabla h(X)=\eta(X) \text { where } \eta \text { is the point in }\{k=1\} \text { with } \frac{X}{|X|}=\frac{\nabla k(\eta)}{|\nabla k(\eta)|} \text {. }
$$

From calculus and Euler's formula for 1-homogeneous functions it now follows that if $X \in \mathbb{S}^{n-1}$ then

$$
h(X)=\langle\eta(X), X\rangle=|X|\left\langle\eta(X), \frac{\nabla k(\eta)}{|\nabla k(\eta)|}\right\rangle=\frac{|X|}{|\nabla k(\eta)|} .
$$

Using this equality we obtain first

$$
\nabla k(\nabla h(X))=\frac{|\nabla k(\eta)| X}{|X|}=\frac{X}{h(X)}
$$

and second using 1-homogeneity of $k, h$ as well as 0 -homogeneity of $\nabla k, \nabla h$, that

$$
k[h(X) \nabla h(X)] \nabla k[h(X) \nabla h(X)]=h(X) k[\nabla h(X)](X / h(X))=X .
$$

Thus $k \nabla k$ and $h \nabla h$ are inverses of each other on $\mathbb{R}^{n} \backslash\{0\}$.

For fixed $p, n<p<\infty, \xi=(p-n) /(p-1)$, and for $X \in \mathbb{R}^{n} \backslash\{0\}$ we define

$$
\hat{\mathcal{F}}(X)= \begin{cases}(h(X))^{\xi} & \text { when } n<p<\infty \\ \log h(X) & \text { when } p=n\end{cases}
$$

We claim that $\hat{\mathcal{F}}$ is a constant multiple of the fundamental solution for $\mathcal{A}$ in (6.14) in the sense of Definition 4.1. Indeed, if $X \in \mathbb{R}^{n} \backslash\{0\}$, it follows from (6.16)-(6.17) that

$$
\begin{aligned}
(\nabla f)(\nabla \hat{\mathcal{F}}(X)) & =p k^{p-1}(\nabla \hat{\mathcal{F}}(X))(\nabla k(\nabla \hat{\mathcal{F}}(X))) \\
& =p \frac{X}{h(X)} k^{p-1}(\nabla \hat{\mathcal{F}}(X)) \\
& =p X \xi^{p-1} h^{[(\xi-1)(p-1)-1]}(X) k^{p-1}(\nabla h(X)) \\
& =p X \xi^{p-1} h^{-n}(X)
\end{aligned}
$$

when $n<p<\infty$. If $p=n$, then using (6.16)-(6.17), we also have

$$
\begin{aligned}
(\nabla f)(\nabla \hat{\mathcal{F}}(X)) & =n k^{n-1}(\nabla \hat{\mathcal{F}}(X))(\nabla k(\nabla \hat{\mathcal{F}}(X))) \\
& =n \frac{X}{h(X)} k^{n-1}(\nabla \hat{\mathcal{F}}(x)) \\
& =n \frac{X}{h(X)} h^{1-n}(X) k^{n-1}(\nabla h(X)) \\
& =n X h^{-n}(X) .
\end{aligned}
$$

In both cases when $n<p<\infty$ and $p=n$ we have that the right hand side in (6.18) and (6.19) are a constant times $X h^{-n}(X)$. Now $X \mapsto h^{-n}(X /|X|)$ is homogeneous of degree 0 so

$$
\left\langle X, \nabla\left[h^{-n}(X /|X|)\right]\right\rangle=0
$$


by Euler's formula. From this observation and (6.18) when $n<p<\infty$ and (6.19) when $p=n$ we deduce

$$
\begin{aligned}
\nabla \cdot((\nabla f)(\nabla \hat{\mathcal{F}}(X))) & =h^{-n}(X /|X|) \nabla \cdot\left(X|X|^{-n}\right)+|X|^{-n}\left\langle X, \nabla\left[h^{-n}(X /|X|)\right]\right\rangle \\
& =0
\end{aligned}
$$

whenever $X \in \mathbb{R}^{n} \backslash\{0\}$ and for fixed $p, n \leq p<\infty$. Hence $\hat{\mathcal{F}}$ is $\mathcal{A}=\nabla f$-harmonic in $\mathbb{R}^{n} \backslash\{0\}$. Also from (6.18) when $n<p<\infty$ and (6.19) when $p=n$ we note that

$$
|\nabla f(\nabla \hat{\mathcal{F}}(X))| \approx|X|^{1-n} \quad \text { on } \mathbb{R}^{n} \backslash\{0\} .
$$

If $\theta \in C_{0}^{\infty}\left(\mathbb{R}^{n}\right)$ then from the above display we deduce that the function $X \mapsto$ $\langle\nabla f(\nabla \hat{\mathcal{F}}(X)), \nabla \theta(X)\rangle$ is integrable on $\mathbb{R}^{n}$. Using this fact, smoothness of $f, h$, and an integration by parts, we get

$$
\begin{aligned}
\int_{\mathbb{R}^{n}}\langle\nabla f(\nabla \hat{\mathcal{F}}(X)), \nabla \theta(X)\rangle d x & =-\lim _{r \rightarrow 0} \int_{\partial B(0, r)} \theta(X)\langle\nabla f(\nabla \hat{\mathcal{F}}(X)), X /|X|\rangle d \mathcal{H}^{n-1} \\
& =C \theta(0) .
\end{aligned}
$$

Using (6.18) once again when $n<p<\infty$ it follows that

$$
\begin{aligned}
C & =-\lim _{r \rightarrow 0} \int_{\partial B(0, r)}\langle\nabla f(\nabla \hat{\mathcal{F}}(X)), X /|X|\rangle d \mathcal{H}^{n-1}=p \xi^{p-1} \int_{\partial B(0,1)} h^{-n}(X /|X|) d \mathcal{H}^{n-1} \\
& =p \xi^{p-1} \int_{\mathbb{S}^{n-1}} h^{-n}(\omega) d \omega .
\end{aligned}
$$

While when $p=n$ we use (6.19) to get

$$
\begin{aligned}
C & =-\lim _{r \rightarrow 0} \int_{\partial B(0, r)}\langle\nabla f(\nabla \hat{\mathcal{F}}(X)), X /|X|\rangle d \mathcal{H}^{n-1}=n \int_{\partial B(0,1)} h^{-n}(X /|X|) d \mathcal{H}^{n-1} \\
& =n \int_{\mathbb{S}^{n-1}} h^{-n}(\omega) d \omega .
\end{aligned}
$$

Remark 6.3. In view of (6.20) and (6.18) when $n<p<\infty$ and (6.19) when $p=n$

$$
\mathcal{F}(x)=C^{\frac{-1}{p-1}} \hat{\mathcal{F}}(x)= \begin{cases}C^{\frac{-1}{p-1}} h(x)^{\xi} & \text { when } n<p<\infty, \\ C^{\frac{-1}{n-1}} \log h(x) & \text { when } p=n\end{cases}
$$

where $\xi=(p-n) /(p-1)$ and $C$ is as in (6.21) when $n<p<\infty$ and as in (6.22) when $p=n$.

Finally using the fact that $k \nabla k$ and $h \nabla h$ are inverses it follows, as in the argument from (7.10) in the proof of Lemma 6.2 of [AGHLV], that for some $\tau>0$, depending only on the data,

$$
\frac{\mathcal{F}_{\omega \omega}(X)}{|\nabla \mathcal{F}(X)|} \geq \tau>0 \quad \text { whenever } \omega, X \in \mathbb{S}^{n-1} \text { with }\langle\nabla \mathcal{F}(X), \omega\rangle=0
$$


To set the stage for our next lemma we now assume that in addition to $\mathcal{A}=\nabla f$ (as in (6.14)) that (2.6) $(i)$ holds. Let $E_{1}$ and $E_{2}$ be convex compact sets containing at least two points and let $\lambda \in(0,1)$ be fixed. Let $\tilde{G} \in\left\{G_{1}, G_{2}, G\right\}$ be the $\mathcal{A}=\nabla f$-harmonic Green's function for $\mathbb{R}^{n} \backslash \tilde{E}$ with pole at infinity whenever $\tilde{E} \in\left\{E_{1}, E_{2}, \lambda E_{1}+(1-\right.$ $\left.\lambda) E_{2}\right\}$. Let $\mathcal{F}$ be the corresponding fundamental solution as in (6.23). Using (6.24) we next show

Lemma 6.4. There exists $R_{1}=R_{1}(\tilde{G}, \alpha, \Lambda, p, n)$, such that if $\tilde{G} \in\left\{G_{1}, G_{2}, G\right\}$, then

$$
\frac{\tilde{G}_{\tilde{\omega} \tilde{\omega}}(x)}{|\nabla \tilde{G}(x)|} \geq \frac{\tau}{2|x|}>0 \quad \text { whenever } \tilde{\omega} \in \mathbb{S}^{n-1} \text { and }|x|>R_{1} \text { with }\langle\nabla \tilde{G}(x), \tilde{\omega}\rangle=0 \text {. }
$$

Proof. The statement and proof of Lemma 6.4 is essentially the same as in Lemma 6.1 of $[\mathrm{AGHLV}]$. It should be noted though that in Lemma $6.1, G$ is the $\mathcal{A}$-harmonic fundamental solution with pole at 0 while $\tilde{u}$ plays the role of the $\mathcal{A}$-harmonic Green's function. Also since the fundamental solution tends to zero at $\infty$ when $1<p<n$ the curvatures on levels of this function are necessarily negative.

To give a brief outline of the proof of Lemma 6.4 , let $\tilde{G}=\mathcal{F}+\tilde{k}$. From Lemmas 4.4 and 5.2 when $p=n$ and Lemmas 4.6 and 5.3 when $p>n$ we see as in (4.54)-(4.56) that $\tilde{k}$ is a weak solution to

$$
\mathcal{L} \tilde{k}:=\sum_{i, j=1}^{n} \frac{\partial}{\partial y_{i}}\left(\bar{a}_{i j}(y) \frac{\partial \tilde{k}}{\partial y_{j}}\right)=0
$$

on $B(x,|x| / 2)$ with $|x| \geq R_{0}$ when $\tilde{E} \subset B\left(0, R_{0} / 2\right)$. Here

$$
\bar{a}_{i j}(y)=\int_{0}^{1} \frac{\partial^{2} f}{\partial \eta_{i} \partial \eta_{j}}(t \nabla \tilde{G}(y)+(1-t) \nabla \mathcal{F}(y)) d t .
$$

Moreover, for some $c \geq 1$, independent of $x$, we also have

$$
c^{-1} \bar{\sigma}(y)|\xi|^{2} \leq \sum_{i, j=1}^{n} \bar{a}_{i j}(y) \xi_{i} \xi_{j} \leq c \bar{\sigma}(y)|\xi|^{2}
$$

whenever $\xi \in \mathbb{R}^{n} \backslash\{0\}$ where $\bar{\sigma}$ satisfies

$$
\bar{\sigma}(y) \approx(|\nabla \bar{G}(y)|+|\nabla \mathcal{F}(y)|)^{p-2} \approx|y|^{\frac{(1-n)(p-2)}{p-1}}
$$

for $|y| \geq R_{0}$. Also from (5.1) (c) when $p=n$ and (5.12) (c) when $n<p<\infty$ we see there exists $\hat{r}_{0}>R_{0}$ and $\beta>0$ such that

$$
|\tilde{k}(y)-\tilde{k}(\infty)| \leq \hat{r}_{0}|y|^{-\beta}
$$

when $|y| \geq \hat{r}_{0}$. Constants depend on various quantities but are independent of $y$ provided $|y| \geq \hat{r}_{0}$. From well-known results for uniformly elliptic PDE (see [GT]) we deduce from (6.26)-(6.28) that

$$
\begin{aligned}
|x|^{-n / 2}\left(\int_{B(x,|x| / 4)}|\nabla \tilde{k}|^{2} d y\right)^{1 / 2} & \leq c|x|^{-1} \max _{B(x,|x| / 2)}|\tilde{k}-\tilde{k}(\infty)| \\
& =O\left(|x|^{-1-\beta}\right) \text { as } x \rightarrow \infty
\end{aligned}
$$


where $c$ as above depends on various quantities but is independent of $x$. Using (6.29), Lemma 6.1, as well as Lemma 3.4 for $\mathcal{F}, \tilde{G}$, and arguing as in (6.8)-(6.15) of [AGHLV], we eventually obtain

$$
|\nabla \tilde{k}|=o\left(|x|^{\frac{1-n}{p-1}}\right) \quad \text { and } \quad \sum_{i, j=1}^{n}\left|\frac{\partial^{2} \tilde{k}}{\partial x_{i} \partial x_{j}}\right|=o\left(|x|^{\frac{2-n-p}{p-1}}\right) \text { as } x \rightarrow \infty .
$$

Now (6.30) and (6.24) imply Lemma 6.4 as in the paragraph following (6.15) of [AGHLV].

We next consider $G^{*}$ defined as earlier in (6.11). If equality holds in either (2.4) when $p=n$ or (2.5) when $p>n$, then using convexity of the domains bounded by the levels of $G, G_{1}, G_{2}$, and repeating the argument above with $u_{1}, u_{2}, u$ replaced by $G_{1}, G_{2}, G$, we see that $G^{*}=G$ and so

$$
\{G(x) \leq t\}=\lambda\left\{G_{1}(y) \leq t\right\}+(1-\lambda)\left\{G_{2}(z) \leq t\right\}
$$

whenever $n \leq p<\infty$ and $t \in(0, \infty)$.

We now use (6.31) and Lemma 6.1 to study the support functions of the convex domains bounded by the levels of $G_{1}, G_{2}$, and $G$. Let $t>0$ be fixed and let $h_{i}(\cdot, t)$ be the support function of $\left\{G_{i} \leq t\right\}$ for $i=1,2$ while $h(\cdot, t)$ is the support function of $\{G \leq t\}$ defined for $X \in \mathbb{R}^{n}$ and $0<t<\infty$ by

$$
h_{i}(X, t):=\sup _{x \in\left\{G_{i} \leq t\right\}}\langle X, x\rangle \text {, for } i=1,2, \quad \text { and } h(X, t):=\sup _{x \in\{G \leq t\}}\langle X, x\rangle .
$$

From the properties of a support function and (6.31) we have

$$
h(X, t)=\lambda h_{1}(X, t)+(1-\lambda) h_{2}(X, t)
$$

whenever $X \in \mathbb{R}^{n}$ and $t>0$.

We note from Lemma 6.1 and Lemma 3.4 that $\nabla \tilde{G} \neq 0$ and $\tilde{G}$ has locally Hölder continuous second partials in $\{\tilde{G}>0\}$ whenever $\tilde{G} \in\left\{G_{1}, G_{2}, G\right\}$. From Lemma 6.4 we see there exists $t_{0}$ large and $\tau_{0}>0$ small and $R_{0}$ large such that if $\tilde{G} \in\left\{G_{1}, G_{2}, G\right\}$ then

$$
\begin{aligned}
& (\star) \quad\{\tilde{G} \geq t\} \subset \mathbb{R}^{n} \backslash \bar{B}\left(0, R_{0}\right) \text { for } 0<t_{0}<t, \\
& (\star \star) \quad \frac{\tilde{G}_{\tilde{\omega} \tilde{\omega}}(x)}{|\nabla \tilde{G}(x)|} \geq \tau_{0} /|x| \text { whenever } \tilde{\omega} \in \mathbb{S}^{n-1} \text { and }|x| \geq R_{0} \text { with }\langle\nabla \tilde{G}(x), \tilde{\omega}\rangle=0 .
\end{aligned}
$$

Hence we conclude from (6.33) that the curvatures at points on $\{\tilde{G}=t\}$ are bounded away from 0 when $t \geq t_{0}$ and $\tilde{G} \in\left\{G_{1}, G_{2}, G\right\}$. This yields

$$
\frac{\nabla \tilde{G}}{|\nabla \tilde{G}|} \text { is a } 1-1 \text { mapping from }\{\tilde{G}=t\} \text { onto } \mathbb{S}^{n-1}
$$

and

$$
\left(\frac{\nabla \tilde{G}}{|\nabla \tilde{G}|}, \tilde{G}\right) \text { is a } 1-1 \text { mapping from }\left\{\tilde{G}>t_{0}\right\} \text { onto } \mathbb{S}^{n-1} \times\left(t_{0}, \infty\right) \text {. }
$$


It follow from (6.33) and the inverse function theorem that if $\tilde{h}$ is the support function corresponding to $\tilde{G} \in\left\{G_{1}, G_{2}, G\right\}$ and $t_{0}<t<\infty$, then $\tilde{h}$ has Hölder continuous second partials in $X$ and

$$
\nabla_{X} \tilde{h}(X, t)=\tilde{x}(X, t) \in\left\{x_{1}(X, t), x_{2}(X, t), x(X, t)\right\}
$$

where $\tilde{x}$ is the point in $\{\tilde{G}=t\}$ with

$$
\frac{X}{|X|}=\frac{\nabla \tilde{G}(\tilde{x})}{|\nabla \tilde{G}(\tilde{x})|}
$$

We now repeat the argument from (6.22) to (6.42) in [AGHLV] to eventually conclude for fixed $t>t_{0}$ and all $X \in \mathbb{S}^{n-1}$ that

$$
\frac{\partial}{\partial X_{i}}\left(\frac{\left|\nabla G_{2}\right|\left(x_{1}\right)}{\left|\nabla G_{1}\right|\left(x_{2}\right)}\right)=0 \text { and } \frac{\partial}{\partial X_{i}}\left(x_{1}-x_{2} \frac{\left|\nabla G_{2}\right|\left(x_{1}\right)}{\left|\nabla G_{1}\right|\left(x_{2}\right)}\right)=0 .
$$

Since $x_{1}, x_{2}$ are smooth for every fixed $t$, there exists $a=a(t), b=b(t) \in \mathbb{R}$ with

$$
x_{2}(X, t)=a x_{1}(X, t)+b \quad \text { whenever } X \in \mathbb{S}^{n-1} .
$$

It now follows from uniqueness of the $\mathcal{A}$-harmonic Green's function in Lemmas 5.2, 5.3, and Remark 2.3 for $\mathcal{A}$-harmonic functions that

$$
G_{2}(x)=G_{1}(a x+b) \quad \text { whenever } G_{2}(x)>t \text { and } t>t_{0} .
$$

Next from Lemma 6.1, (3.3), homothetic invariance of $\mathcal{A}$-harmonic functions, and the same argument as in (4.54)-(4.56) or (6.25)-(6.27) we see that $G_{2}(x)-G_{1}(a x+b)$ is a solution to a locally uniform elliptic divergence form PDE with Lipschitz coefficients. This fact and a unique continuation theorem in [GL] imply as after (6.44) in [AGHLV] that the above equality holds whenever $x \in \mathbb{R}^{n} \backslash E_{2}$ or equivalently that $E_{1}=a E_{2}+b$. The proof of Theorem A is now complete.

\section{Part 2. A Minkowski problem for $\mathcal{A}$-harmonic Green's function}

\section{InTRODUCTION AND STATEMENT OF RESULTS}

In this section we study the Minkowski problem associated with an $\mathcal{A}=\nabla f$ harmonic Green's function with pole at $\infty$ when $f$ is as in Theorem A. To be more specific, suppose $E \subset \mathbb{R}^{n}$ is a compact convex set with nonempty interior. Then for $\mathcal{H}^{n-1}$ almost every $x \in \partial E$, there is a well defined outer unit normal, $\mathbf{g}(x, E)$ to $\partial E$. The function $\mathbf{g}(\cdot, E): \partial E \mapsto \mathbb{S}^{n-1}$ (whenever defined) is called the Gauss map for $\partial E$. Let $\mu$ be a finite positive Borel measure on $\mathbb{S}^{n-1}$ satisfying

$$
\begin{aligned}
& \text { (i) } \int_{\mathbb{S}^{n-1}}|\langle\theta, \zeta\rangle| d \mu(\zeta)>0 \quad \text { for all } \theta \in \mathbb{S}^{n-1}, \\
& \text { (ii) } \int_{\mathbb{S}^{n-1}} \zeta d \mu(\zeta)=0 .
\end{aligned}
$$

We prove 
Theorem B. Let $\mu$ be as in (7.1) and $p$ be fixed, $n \leq p<\infty$. Let $\mathcal{A}=\nabla f$ be as in (2.6) and Definition 2.1. Then there exists a compact convex set $E$ with nonempty interior and $\mathcal{A}$-harmonic Green's function $u$ for $\mathbb{R}^{n} \backslash E$ with a pole at infinity satisfying $(7.2)$

(a) $\lim _{y \rightarrow x} \nabla u(y)=\nabla u(x)$ exists for $\mathcal{H}^{n-1}$-almost every $x \in \partial E$ as $y \in \mathbb{R}^{n} \backslash E$ approaches $x$ non-tangentially.

(b) $\int_{\partial E} f(\nabla u(x)) d \mathcal{H}^{n-1}<\infty$.

(c) $\int_{\mathbf{g}^{-1}(K, E)} f(\nabla u(x)) d \mathcal{H}^{n-1}=\mu(K)$ whenever $K \subset \mathbb{S}^{n-1}$ is a Borel set.

(d) $E$ is the unique set up to translation for which (c) holds.

We remark that Minkowski originally considered a similar problem for surface area measure (in Theorem B omit $(a),(b)$, and replace $f(\nabla u(x))$ in $(c)$ by 1 ). We also mention that Jerison in $[\mathrm{J}]$ proved a Minkowski type theorem similar to the above when $u$ is the Newtonian capacitary function of a compact convex set. His result was generalized in [CNSXYZ] to $p$-harmonic functions when $1<p<2$. In [AGHLV], the first, second, and fourth authors of this article, along with Jasun Gong and Jay Hineman, obtained an analogue of Theorem $\mathrm{B}$ when $1<p<n$ for the $\mathcal{A}=\nabla f$ capacitary function of a compact convex set $E$ with nonempty interior. For more historical details see [AGHLV, section 8].

As a broad outline of our proof of Theorem B, we follow [AGHLV] who in turn used ideas from $[\mathrm{J}]$ and [CNSXYZ]. Part of the preliminary work for the analogue of Theorem B in [AGHLV] involved generalizing work from [LN, LN1, LN2, LN3] for positive $p$-harmonic functions vanishing on a portion of the boundary of a Lipschitz domain to positive $\mathcal{A}=\nabla f$-harmonic functions vanishing on a portion of the boundary of a Lipschitz domain when $1<p<n$. Much of this work extends without change to the $p \geq n$ and $\mathcal{A}=\nabla f$ situation so we shall often refer to Lemmas in [AGHLV] for proofs. This generalization is done in $\S 8$. From our work in $\S 8$ it follows that if $\tilde{E}$ is a compact convex set with non empty interior and if $\tilde{u}$ is the $\mathcal{A}$-harmonic Green's function for $\tilde{E}$ with pole at $\infty$, then $(7.2)(a),(b)$ hold with $u, E$ replaced by $\tilde{u}, \tilde{E}$. The Gauss map and corresponding measure $\tilde{\mu}$ can then be defined relative to $\tilde{u}, \tilde{E}$ as in $(7.2)(c)$

In $\S 9$ we consider a sequence of compact convex sets, say $\left\{\tilde{E}_{m}\right\}_{m \geq 1}$ with nonempty interiors which converge in the sense of Hausdorff distance to $\widetilde{E}$, a compact convex set with 0 in the interior of $\tilde{E}$. For a fixed $\mathcal{A}=\nabla f$ as in Theorem B, let $\tilde{u}_{m}$ for $m=1,2, \ldots$, and $\tilde{u}$ be the $\mathcal{A}$-harmonic Green's functions with pole at $\infty$ for $\mathbb{R}^{n} \backslash \tilde{E}_{m}$ and $\mathbb{R}^{n} \backslash \tilde{E}$ respectively. If $\tilde{\mu}_{m}$ for $m=1,2, \ldots$, and $\tilde{\mu}$ denote the Borel measures corresponding to $\tilde{u}, \tilde{u}_{m}$ as in the above discussion, we show that $\left\{\tilde{\mu}_{m}\right\}$ converges weakly to $\tilde{\mu}$ on $\mathbb{S}^{n-1}$. In $\S 10$ we use this result to derive the Hadamard variational formula for the derivative of $t \rightarrow \mathcal{C}_{\mathcal{A}}\left(t E_{1}+(1-t) E_{2}\right)$ whenever $t \in[0,1)$ 
and $E_{1}, E_{2}$ are compact convex sets with nonempty interiors. Proofs for $p \geq n$ are more delicate than in the case $1<p<n$ considered in [AGHLV], primarily because our $\mathcal{A}$-harmonic Green's function blows up at $\infty$ when $p \geq n$, whereas $\mathcal{A}$ harmonic capacitary functions have limit 0 at $\infty$ when $1<p<n$. In $\S 11$, we give the proof of Theorem B. As in [AGHLV] the proof essentially consists in showing that a certain minimum problem has a solution, say $\tilde{E}$, in the class of compact convex sets with nonempty interior. To rule out the possibility that $\tilde{E}$ has Hausdorff dimension $k \leq n-1$, we argue as in [AGHLV] when $k<n-1$. However if $k=n-1$ we are not able to use the same argument as in the case $1<p<n$. Instead we study positive $\mathcal{A}$ harmonic solutions vanishing on a ray in $\mathbb{R}^{2}$ when $p \geq n$ and use our results from this study to show that $\tilde{E}$ cannot be $n-1$ dimensional. Finally, uniqueness in Theorem $\mathrm{B}$ is proved in the last part of section 11 using Theorem A.

\section{BoundaRY BEHAVIOR OF $\mathcal{A}$-HARMONIC FUnCTIONS IN LipsChitz DOMAINS}

Throughout this and later sections, the data continues to be $p, n, \alpha, \Lambda$. We begin this section with several definitions and lemmas copied from [AGHLV]. Recall that $\phi: K \rightarrow \mathbb{R}$ is said to be Lipschitz on $K$ provided there exists $\hat{b}, 0<\hat{b}<\infty$, such that

$$
|\phi(z)-\phi(w)| \leq \hat{b}|z-w| \quad \text { whenever } z, w \in K \text {. }
$$

The infimum of all $\hat{b}$ such that (8.1) holds is called the Lipschitz norm of $\phi$ on $K$, denoted $\| \phi \hat{\|}_{K}$. It is well-known that if $K \subset \mathbb{R}^{n-1}$ is compact, then $\phi$ has an extension to $\mathbb{R}^{n}$ (also denoted $\phi$ ) which is differentiable almost everywhere in $\mathbb{R}^{n}$, and

$$
\left\|\phi \hat{\|}_{\mathbb{R}^{n-1}}=\right\||\nabla \phi|\left\|_{\infty} \leq c\right\| \phi \hat{\|}_{K}
$$

Now suppose that $D$ is an open set, $w \in \partial D$, and

$$
\begin{aligned}
\partial D \cap B(w, 4 \hat{r}) & =\left\{y=\left(y^{\prime}, y_{n}\right) \in \mathbb{R}^{n}: y_{n}=\phi\left(y^{\prime}\right)\right\} \cap B(w, 4 \hat{r}), \\
D \cap B(w, 4 \hat{r}) & =\left\{y=\left(y^{\prime}, y_{n}\right) \in \mathbb{R}^{n}: y_{n}>\phi\left(y^{\prime}\right)\right\} \cap B(w, 4 \hat{r})
\end{aligned}
$$

in an appropriate coordinate system for some Lipschitz function $\phi$ on $\mathbb{R}^{n-1}$. Note from elementary geometry that if $\zeta \in \partial D \cap B(w, 2 \hat{r})$ and $0<s<\hat{r}$, we can find points

$$
a_{s}(\zeta) \in D \cap B(\zeta, s) \text { with } \quad d\left(a_{s}(\zeta), \partial D\right) \geq c^{-1} s
$$

for a constant $c$ depending on $\| \nabla \phi \hat{\|}$. In the following, we let $a_{s}(\zeta)$ denote one such point. Also if $\zeta \in \partial D \cap B(w, 2 \hat{r})$, and $t>1$ let

$$
\Gamma(\zeta)=\Gamma(\zeta, t)=\{y \in D \cap B(w, 4 \hat{r}):|y-\zeta|<t d(y, \partial D)\} .
$$

Unless otherwise stated we always assume $t$ is fixed and so large that $\Gamma(\zeta)$ contains the inside of a truncated cone with vertex at $\zeta$, height $\hat{r}$, axis along the positive $e_{n}$ axis, and of angle opening $\theta=\theta(t)>0$. Given a measurable function $g$ on $D \cap B(w, 4 r)$, where $0<r<\hat{r}$, put $\Delta(w, r)=\partial D \cap B(w, r)$ and define the non-tangential maximal function

$$
\mathcal{N}_{r}(g): \Delta(w, r) \rightarrow \mathbb{R}
$$


of $g$ relative to $D \cap B(w, 4 r)$ by

$$
\mathcal{N}_{r}(g)(x)=\sup _{y \in \Gamma(x) \cap B(w, 4 r)}|g|(y) \quad \text { whenever } x \in \Delta(w, r) .
$$

Next we note as in Lemmas 3.5 and 3.7:

Lemma 8.1. Let $D, w, \hat{r}, \phi$ be as in (8.2) and $1<p<\infty$. Suppose $w \in \partial D, 0<4 r<$ $\hat{r}$, and $v$ is a positive $\mathcal{A}$-harmonic function in $D \cap B(w, 4 r)$ with $v \equiv 0$ on $\partial D \cap B(w, 4 r)$ in the $W^{1, p}$ Sobolev sense. Then $v$ has a representative in $W^{1, p}(D \cap B(w, s)), s<4 r$ which extends to a Hölder continuous function on $B(w, s)$ (denoted also by $v$ ) with $v \equiv 0$ on $B(w, s) \backslash D$. Also, there exists $\bar{c} \geq 1$, depending only on the data and $\| \phi \hat{\|}$, such that if $\bar{r}=r / \bar{c}$, then

$$
\bar{r}^{p-n} \int_{B(w, \bar{r})}|\nabla v|^{p} d x \leq \bar{c}\left(v\left(a_{2 \bar{r}}(w)\right)\right)^{p} .
$$

Moreover, there exists $\hat{\sigma} \in(0,1)$, depending only on the data and $\| \phi \hat{\|}$, such that

$$
|v(x)-v(y)| \leq \bar{c}\left(\frac{|x-y|}{\bar{r}}\right)^{\hat{\sigma}} v\left(a_{2 \bar{r}}(w)\right) \quad \text { whenever } x, y \in B(w, \bar{r}) .
$$

Finally there exists a unique finite positive Borel measure $\tau$ on $\mathbb{R}^{n}$, with support contained in $\bar{\Delta}(w, r)$, such that

$$
\begin{aligned}
& \text { (a) } \int\langle\nabla f(\nabla v), \nabla \psi\rangle d x=-\int \psi d \tau \quad \text { whenever } \psi \in C_{0}^{\infty}(B(w, r)), \\
& \text { (b) } \bar{c}^{-1} \bar{r}^{p-n} \tau(\Delta(w, \bar{r})) \leq\left(v\left(a_{2 \bar{r}}(w)\right)\right)^{p-1} \leq \bar{c} \bar{r}^{p-n} \tau(\Delta(w, \bar{r})) .
\end{aligned}
$$

Next we state as lemmas some results given for starlike Lipschitz domains in Lemma 9.5, Proposition 9.7, Lemma 10.9, and Corollary 10.10 of [AGHLV] when $1<p<n$. For the definition of a starlike Lipschitz domain and justification for using these results in the present setting, see Definition 8.4 and the discussion following this definition.

Lemma 8.2. Let $D, w, \hat{r}, r, \phi, p, v, \tau$ be as in Lemma 8.1. There exists $c_{\star} \geq 1$, depending only on the data and $\|\phi\| \hat{~ s u c h ~ t h a t ~ i f ~} 4 \tilde{r}=r / c_{\star}$ and $x \in B(w, \tilde{r}) \cap D$, then

$$
\begin{aligned}
& \text { (a) } c_{\star}^{-1} \frac{v(x)}{d(x, \partial D)} \leq\left\langle\nabla v(x), e_{n}\right\rangle \leq|\nabla v(x)| \leq c_{\star} \frac{v(x)}{d(x, \partial D)}, \\
& \text { (b) } \lim _{\substack{x \rightarrow y \\
x \in \Gamma(y) \cap B(w, 2 r)}} \nabla v(x) \stackrel{\text { def }}{=} \nabla v(y) \text { exists for } \mathcal{H}^{n-1} \text {-almost every } y \in \Delta(w, \tilde{r}) .
\end{aligned}
$$

Moreover, $\Delta(w, \tilde{r})$ has a tangent plane for $\mathcal{H}^{n-1}$-almost every $y \in \Delta(w, \tilde{r})$. If $\mathbf{n}(y)$ denotes the unit normal to this tangent plane pointing into $D \cap B(w, 2 \tilde{r})$, then

$$
\nabla v(y)=|\nabla v(y)| \mathbf{n}(y) \quad \text { for } \mathcal{H}^{n-1} \text {-almost every } y \in \Delta(w, 2 \tilde{r})
$$

and

$$
\frac{d \tau}{d \mathcal{H}^{n-1}}(y)=p \frac{f(\nabla v(y))}{|\nabla v(y)|} \quad \text { for } \mathcal{H}^{n-1} \text {-almost every } y \in \Delta(w, 2 \tilde{r})
$$


Finally, there exists $q>p /(p-1)$ and $c_{\star \star}$ with the same dependence as $c_{\star}$ such that

$$
\begin{aligned}
& \text { (a) } \int_{\Delta(w, \tilde{r})}\left(\frac{f(\nabla v)}{|\nabla v|}\right)^{q} d \mathcal{H}^{n-1} \leq c_{\star \star} r^{(n-1)(1-q)}\left(\int_{\Delta(w, \tilde{r})} \frac{f(\nabla v)}{|\nabla v|} d \mathcal{H}^{n-1}\right)^{q} . \\
& \text { (b) } \int_{\Delta(w, \tilde{r})} \mathcal{N}_{\tilde{r}}(\nabla v)^{q(p-1)} d \mathcal{H}^{n-1} \leq c_{\star \star} r^{(n-1)(1-q)}\left(\int_{\Delta(w, \tilde{r})} \mathcal{N}_{\tilde{r}(\nabla v)^{(p-1)}} d \mathcal{H}^{n-1}\right)^{q} .
\end{aligned}
$$

Lemma 8.3. Let $D, w, \hat{r}, r, p$, be as in Lemma 8.1. Also let $v_{i}$, for $i=1,2$ be as in this lemma with $v$ replaced by $v_{i}$. There exist $\alpha_{+} \in(0,1)$ and $c_{+} \geq 1$, depending only on the data and $\| \phi \hat{\|}$, such that if $r^{+}=r / c^{+}$then

$$
\left|\frac{v_{1}(x)}{v_{2}(x)}-\frac{v_{1}(y)}{v_{2}(y)}\right| \leq c_{+}\left(\frac{|x-y|}{r^{+}}\right)^{\alpha+} \frac{v_{1}\left(a_{r^{+}}(w)\right)}{v_{2}\left(a_{r^{+}}(w)\right)}
$$

whenever $x, y \in D \cap B\left(w, 2 r^{+}\right)$.

To outline the proof of these lemmas we need a definition.

Definition 8.4 (Starlike Lipschitz domain). A bounded domain $D \subset \mathbb{R}^{n}$ is said to be starlike Lipschitz with respect to $z \in D$ provided

$$
\begin{aligned}
& \partial D=\{z+\mathcal{R}(\omega) \omega: \omega \in \partial B(0,1)\} \\
& \text { where } \log \mathcal{R}: \partial B(0,1) \rightarrow \mathbb{R} \text { is Lipschitz on } \partial B(0,1) .
\end{aligned}
$$

Under the above scenario we say that $z$ is the center of $D$ and $\|\log \mathcal{R}\|_{\mathbb{S}^{n-1}}$ is the starlike Lipschitz constant for $D$. We note that if $D, w, \phi$, and $\hat{r}$ are as in (8.2), $0<4 r<\hat{r}$ and $w^{\prime}=w+r e_{n} / 2$, then there exists $c^{\prime} \geq 100$, depending only on $\| \phi \hat{\|}$, such that the following is true: Let $r^{\prime}=r / c^{\prime}$ and let $D^{\prime}$ denote the interior of the set obtained from the union of all line segments connecting points in $\partial D \cap B\left(w, r^{\prime}\right)$ to points in $B\left(w^{\prime}, r^{\prime}\right)$. Then $D^{\prime}$ is starlike Lipschitz with respect to $w^{\prime}$. Moreover, if $\mathcal{R}^{\prime}$ is the graph function for $D^{\prime}$, then

$$
\| \log \mathcal{R}^{\prime} \hat{\|}_{\mathbb{S}^{n-1}} \leq c^{\prime}(\| \phi \hat{\|}+1) .
$$

Also if $D^{\prime}$ is a starlike Lipschitz domain with center at $w^{\prime}$, graph function $\mathcal{R}^{\prime}, w \in \partial D^{\prime}$, $\frac{w^{\prime}-w}{\left|w^{\prime}-w\right|}=e_{n}$, and $10^{-2} d\left(w^{\prime}, \partial D^{\prime}\right)<s<10^{-1} d\left(w^{\prime}, \partial D^{\prime}\right)$, then there exists $c^{\prime \prime} \geq 1$, depending only on $\| \log \mathcal{R}^{\prime} \hat{\|}_{\mathbb{S}^{n-1}}$, such that if $s^{\prime}=s / c^{\prime \prime}$ then

$$
\partial D^{\prime} \cap B\left(w, s^{\prime}\right)=\partial D^{\prime} \cap\left\{\left(y^{\prime}, \phi^{\prime}\left(y^{\prime}\right)\right\} \quad \text { and } \quad D^{\prime} \cap B\left(w, s^{\prime}\right)=D^{\prime} \cap\left\{y: y_{n}>\phi^{\prime}\left(y^{\prime}\right)\right\}\right.
$$

where $\phi^{\prime}$ is Lipschitz on $\mathbb{R}^{n-1}$ and

$$
\left\|\phi^{\prime}\right\| \leq c^{\prime \prime}\left(\mid \log \mathcal{R}^{\prime} \|_{\mathbb{S}^{n-1}}+1\right) .
$$

In $[A G H L V]$ results analogous to Lemmas 8.2, 8.3 were first proved for $1<p<n$ when $D$ is a starlike Lipschitz domain. The results obtained were later used as in (8.11), to prove similar results in the Lipschitz graph setting (see Lemma 10.11 in [AGHLV]). To briefly outline the proofs given in [AGHLV] for $1<p<n$ and starlike Lipschitz domains, we note that $(8.6)(b),(8.7),(8.8),(8.9)$, were proved in [AGHLV] 
under the assumption that an analogue of (8.6) (a) holds for $v$. This was done in Lemmas 9.5, 9.6, and Proposition 9.7. In section 10 of [AGHLV] the authors used the results in Proposition 9.7 to state and prove some rather difficult estimates for a certain elliptic measure in Lemmas 10.1-10.3. In Lemmas 10.4, 10.5, the authors define and study the $\mathcal{A}$-harmonic Green's function, say $G^{\prime}$ for a starlike Lipschitz domain, $D^{\prime}$, with pole at the center, $w^{\prime}$, of this domain. They obtain an analogue of (8.6) (a) for $v=G^{\prime}$ when $1<p<n$. These results are then used in Lemma 10.7 to show that the ratio of $v_{1} / v_{2}$, in the analogue of Lemma 8.3 for starlike Lipschitz domains, is at least bounded. Finally using a perturbation type argument in Lemma 10.9 and Corollary 10.10 they eventually get their version of Lemma 8.3 for $1<p<n$ and essentially simultaneously (8.6) $(a)$ for $v_{1}, v_{2}$. This result is then restated for Lipschitz domains in Lemma 10.11 of [AGHLV] for $1<p<n$.

The proof of Lemmas 10.4, 10.5, in [AGHLV] required a somewhat lengthy study of the Green's function, which however was also used (see Lemma 13.7 in [AGHLV]) to prove the important Proposition 13.6 of that paper. If $p>n$ this approach can no longer be used to get an analogue of Proposition 13.6 in [AGHLV]. There are also certain questions which we do not want to consider when $p>n$, such as should $G^{\prime}$ have a positive point mass (in which case $G^{\prime} \leq 0$ ), or a negative point mass at $w^{\prime}$. To avoid these deliberations, one can replace $G^{\prime}$ in the above proof scheme for fixed $p \geq n$, by the $\mathcal{A}$-harmonic function $v^{\prime}$ in $D^{\prime} \backslash \bar{B}\left(w^{\prime}, s^{\prime}\right)$ with continuous boundary values: $v^{\prime} \equiv 1$ on $\partial B\left(w^{\prime}, s^{\prime}\right)$ and $v^{\prime} \equiv 0$ on $\partial D^{\prime}$. Here $D^{\prime}$ is a starlike Lipschitz domain with center at $w^{\prime}$ and graph function $\mathcal{R}^{\prime}$. Also $s^{\prime}=d\left(w^{\prime}, \partial D^{\prime}\right) / c^{\prime}$ where $c^{\prime} \geq 100$ is fixed. We prove

Lemma 8.5. Let $p, D^{\prime}, v^{\prime}, w^{\prime}, s^{\prime}$ be as above. There exists $c \geq 1$ depending only on the data, $c^{\prime}$, and $\| \log \mathcal{R}^{\prime} \hat{\|}_{\mathbb{S}^{n-1}}$ such that

$$
\begin{aligned}
& \text { (a) } \quad 0<\left|\nabla v^{\prime}(x)\right| \leq c\left\langle\frac{w^{\prime}-x}{\left|w^{\prime}-x\right|}, \nabla v^{\prime}(x)\right\rangle \quad \text { whenever } x \in D^{\prime} \backslash \bar{B}\left(w^{\prime}, s^{\prime}\right) . \\
& \text { (b) } \quad c^{-1} \frac{v^{\prime}(x)}{d\left(x, \partial D^{\prime}\right)} \leq\left|\nabla v^{\prime}(x)\right| \leq c \frac{v^{\prime}(x)}{d\left(x, \partial D^{\prime}\right)} \quad \text { whenever } x \in D^{\prime} \backslash \bar{B}\left(w^{\prime}, 2 s^{\prime}\right) .
\end{aligned}
$$

Proof. The proof of this lemma is essentially the same as Lemma 10.5 in [AGHLV] however since we are using a different function we give some details. To start the proof we assume, as we may, (since $\mathcal{A}$-harmonic functions are invariant under translation and dilation and (8.13) is also invariant under translation and dilation) that

$$
w^{\prime}=0 \text { and } \operatorname{diam}\left(D^{\prime}\right)=1 .
$$

Using Lipschitz starlikeness of $\partial D^{\prime}$, the maximum principle for $\mathcal{A}$-harmonic functions, and arguing as in the proof of (4.6) we find for some $\tilde{c} \geq 1$ and $\lambda>1$ near 1 , that

$$
\frac{v^{\prime}(x)-v^{\prime}(\lambda x)}{\lambda-1} \geq \frac{v^{\prime}(x)}{\tilde{c}} \quad \text { whenever } x \in D^{\prime} \backslash \bar{B}\left(0, s^{\prime}\right)
$$

where $\tilde{c}$ depends only on the data, $c^{\prime}$ and $\| \log \mathcal{R}^{\prime} \hat{\|}_{\mathbb{S}^{n-1}}$. Letting $\lambda \rightarrow 1$ we obtain

$$
-\tilde{c}\left\langle\nabla v^{\prime}(x), x\right\rangle \geq v^{\prime}(x) \quad \text { whenever } x \in D^{\prime} \backslash \bar{B}\left(0, s^{\prime}\right) .
$$


To get estimates near $\partial D^{\prime}$, let

$$
\mathcal{P}(x)=-\left\langle\nabla v^{\prime}(x), x\right\rangle \quad \text { whenever } x \in D^{\prime} \backslash \bar{B}\left(0, s^{\prime}\right) .
$$

As in (4.13) we note that $\psi=v_{x_{i}}^{\prime}$ for $1 \leq i \leq n, \psi=v^{\prime}$, and $\psi=\mathcal{P}$ are all weak solutions in $D^{\prime} \backslash B\left(0, s^{\prime}\right)$ to

$$
\sum_{i, j=1}^{n} \frac{\partial}{\partial x_{i}}\left(\hat{b}_{i j} \psi_{x_{j}}\right)=0
$$

where

$$
\hat{b}_{i j}(x)=f_{\eta_{i} \eta_{j}}\left(\nabla v^{\prime}(x)\right) \quad \text { whenever } \quad x \in D^{\prime} \backslash \bar{B}\left(0, s^{\prime}\right) .
$$

We temporarily assume that $\mathcal{R}^{\prime}$ has an extension to $\mathbb{R}^{n}$ (also denoted $\mathcal{R}^{\prime}$ ) with

$$
\mathcal{R}^{\prime} \in C^{\infty}\left(\mathbb{R}^{n}\right) \text {. }
$$

Then from a theorem of Lieberman in [Li] we deduce that $\mathcal{P}$ and $v_{x_{i}}^{\prime}$ for $1 \leq i \leq n$ have continuous extensions to the closure of $D^{\prime} \backslash B\left(0, s^{\prime}\right)$. Using Lipschitz starlikeness of $D^{\prime}$, and (8.14) we find for some $\breve{c} \geq 1$ depending only on the data, $c^{\prime}$, and $\| \log \mathcal{R}^{\prime} \hat{\|}_{\mathbb{S}^{n-1}}$ that

$$
\breve{c} \mathcal{P}(x) \geq \pm v_{x_{i}}^{\prime}(x) \quad \text { on } \quad \partial D^{\prime} \cup \partial B\left(0, s^{\prime}\right)
$$

when $1 \leq i \leq n$. From this inequality and the boundary maximum principle for the PDE in (8.15), we conclude that (8.13) $(a)$ is valid when (8.17) holds with constants depending only on the data, $c^{\prime}$, and $\| \log \mathcal{R}^{\prime} \hat{\|}_{\mathbb{S}^{n-1}}$. As for (8.13) (b) the right-hand inequality in this display follows from $(3.2)(\hat{a})$. Thus we prove only the left-hand inequality in (8.13) (b). To accomplish this if $x \in D^{\prime} \backslash \bar{B}\left(0,2 s^{\prime}\right)$, we draw a ray $l$ from 0 through $x$ to a point in $\partial D^{\prime}$. Let $y$ be the first point on $l$ (starting from $x$ ) with $v^{\prime}(y)=v^{\prime}(x) / 2$. Then from elementary calculus there exists $\hat{w}$ on the part of $l$ between $x, y$ with

$$
v^{\prime}(x) / 2=v^{\prime}(x)-v^{\prime}(y) \leq\left|\nabla v^{\prime}(\hat{w})\right||y-x| .
$$

From (8.4) we deduce the existence of $c \geq 1$ depending only on the data, $c^{\prime}$, and $\left\|\log \mathcal{R}^{\prime}\right\|_{\mathbb{S}^{n-1}}$ with

$$
y, \hat{w} \in B\left[x,\left(1-c^{-1}\right) d\left(x, \partial D^{\prime}\right)\right] .
$$

Using (8.19), Harnack's inequality for $\mathcal{P}$, and (8.13)(a), it follows for some $c$, depending only on the data, $c^{\prime}$, and $\| \log \mathcal{R}^{\prime} \hat{\|}_{\mathbb{S}^{n-1}}$, that

$$
\left|\nabla v^{\prime}(\hat{w})\right| \leq c\left|\nabla v^{\prime}(x)\right| \text {. }
$$

This inequality, (8.18), and (8.19) imply that

$$
v^{\prime}(x) \leq c\left|\nabla v^{\prime}(x)\right| d\left(x, \partial D^{\prime}\right) .
$$

We conclude that the left-hand inequality in (8.13) (b) is valid for $x \in D^{\prime} \backslash \bar{B}\left(0,2 s^{\prime}\right)$ when $c$ is suitably large and (8.17) holds. To remove this assumption choose $\mathcal{R}_{m} \in$ $C^{\infty}\left(\mathbb{R}^{n}\right)$ for $m=1,2, \ldots$, with

$$
\left\|\log \mathcal{R}_{m} \hat{\|}_{\mathbb{S}^{n-1}} \leq c\right\| \log \mathcal{R}^{\prime} \hat{\|}_{\mathbb{S}^{n-1}}
$$


and $\mathcal{R}_{m} \rightarrow \mathcal{R}^{\prime}$ as $m \rightarrow \infty$ uniformly on $\mathbb{S}^{n-1}$. Here $c$ depends only on $n$. Let $\left(D_{m}\right)$ be the corresponding sequence of starlike Lipschitz domains and for large $m$, let $v_{m}$ be the $\mathcal{A}$-harmonic function in $D_{m} \backslash \bar{B}\left(0, s^{\prime}\right)$ with $v_{m} \equiv 1$ on $\partial B\left(0, s^{\prime}\right)$ and $v_{m} \equiv 0$ on $\partial D_{m}$. Using Lemmas 3.4 and 8.1, we see that

$$
\begin{aligned}
\left\{v_{m}, \nabla v_{m}\right\} & \text { converge to }\left\{v^{\prime}, \nabla v^{\prime}\right\} \\
& \text { uniformly on compact subsets of } D^{\prime} \backslash \bar{B}\left(0, s^{\prime}\right) .
\end{aligned}
$$

Applying Lemma 8.5 to $v_{m}$, and using the fact that the constants in this lemma are independent of $m$ we conclude upon taking limits that Lemma 8.5 holds for $v^{\prime}$ without hypothesis (8.17).

Other than substituting Lemma 8.5 for Lemmas 10.4, 10.5 in [AGHLV], the proof in [AGHLV], outlined above, can also be used if $p \geq n$ to prove Lemmas 8.2, 8.3. Thus we omit further details in the proofs of these lemmas.

For use in later sections we make the following definition:

Definition 8.6 (Lipschitz domain). A domain $D \subset \mathbb{R}^{n}$ is called a bounded Lipschitz domain provided there exists a finite set of balls $\left\{B\left(x_{i}, r_{i}\right)\right\}$ covering an open neighborhood of $\partial D$, such that (8.2) holds with $r_{i}=\hat{r}$ and $\phi_{i}=\phi$. The Lipschitz constant of $D$ is defined to be $M=\max _{i}\left\|\left|\nabla \phi_{i}\right|\right\|_{\infty}$.

\section{WEAK CONVERGENCE OF CERTAIN MEASURES ON $\mathbb{S}^{n-1}$}

Let $E$ be a compact convex set with 0 in the interior of $E, p \geq n$, and let $u$ be the $\mathcal{A}$-harmonic Green's function for $\mathbb{R}^{n} \backslash E$ with pole at infinity. We note that $\mathbb{R}^{n} \backslash E$ is a Lipschitz domain so Lemma 8.2 holds with $v=u, D=\mathbb{R}^{n} \backslash E$ provided $\hat{r}>0$ is small enough. From Lemma 8.2 we see that if $\mathbf{g}(x, E)=\mathbf{g}: \partial E \rightarrow \mathbb{S}^{n-1}$ is defined by

$$
\mathbf{g}(x, E)=-\frac{\nabla u(x)}{|\nabla u(x)|}
$$

then this equality is well-defined on a set $\Theta \subset \partial E$ with $\mathcal{H}^{n-1}(\partial E \backslash \Theta)=0$. Also from Lemma 8.2 we see that if $F \subset \mathbb{S}^{n-1}$ is a Borel set, then $\mathbf{g}^{-1}(F, E)$ is $\mathcal{H}^{n-1}$ measurable. Define a measure $\mu(\cdot)=\mu_{E, f}(\cdot)$ on $\mathbb{S}^{n-1}$ by

$$
\mu(F):=\int_{\Theta \cap \mathbf{g}^{-1}(F, E)} f(\nabla u) d \mathcal{H}^{n-1} \text { whenever } F \subset \mathbb{S}^{n-1} \text { is a Borel set. }
$$

Next suppose that $\left\{E_{m}\right\}_{m \geq 1}$ is a sequence of compact convex sets with nonempty interiors which converge to $E$ in the sense of Hausdorff distance. That is, $d_{\mathcal{H}}\left(E_{m}, E\right) \rightarrow 0$ as $m \rightarrow \infty$ where $d_{\mathcal{H}}$ was defined at the beginning of section 2 . Let $u_{m}$ be the corresponding $\mathcal{A}=\nabla f$-harmonic Green's function for $\mathbb{R}^{n} \backslash E_{m}$ when $m=1,2, \ldots$, with pole at infinity. Then for $m$ large enough say $m \geq m_{0}$ we see that Lemma 8.2 is valid with $D_{m}=\mathbb{R}^{n} \backslash E_{m}, u_{m}=v$, and $w \in \partial D_{m}$. Here $\hat{r}>0$ can be chosen independent of $m$ and constants depend only on the data as well as the Lipschitz constant for $E$. For fixed $m$, let $\mu_{m}$ be the measure on $\mathbb{S}^{n-1}$ defined as in (9.1) relative to $f, u_{m}$, and $\mathbf{g}\left(\cdot, E_{m}\right)$. We prove 
Proposition 9.1. Fix $p \geq n$ and define $\mu_{m}$ and $\mu$ relative to $E_{m}$ and $E$ respectively as above. Then

$$
\mu_{m} \rightarrow \mu \quad \text { weakly as } m \rightarrow \infty .
$$

Proof. The proof of this proposition is given in [AGHLV, Proposition 11.1] for $1<$ $p<n$. The proof just uses Lemmas 8.1-8.3, although in one place (see the proof of claim 11.9 in [AGHLV]) the authors use $\mathcal{A}$-capacitary functions to construct an $\mathcal{A}$-harmonic function $v$ in $K=K(\hat{\theta})=\left\{x: x_{n}>|x| \cos (\hat{\theta})\right\}$. Here $v$ is continuous in $\mathbb{R}^{n}$ with $v \equiv 0$ on $\mathbb{R}^{n} \backslash K$ for $\hat{\theta}>\pi / 2$, but near $\pi / 2$ satisfying $v\left(e_{n}\right)=1$. To get $v$ in our situation let $v_{m}$ be $\mathcal{A}$-harmonic function in $B(0,2 m) \backslash[\bar{B}(0, m) \backslash K]$ and continuous on $\bar{B}(0,2 m)$ with $v \equiv 0$ on $\bar{B}(0, m) \backslash K$ and $v \equiv a_{m}$ on $\partial B(0,2 m)$ where $a_{m}$ is chosen so that $v_{m}\left(e_{n}\right)=1$. Existence of $v_{m}$ follows from uniform $(2 m, p)$-fatness of $\partial B(0,2 m)$ and $\bar{B}(0, m) \backslash K$. Using Harnack's inequality, Lemmas 8.1 and 3.4, and Ascoli's theorem we see that a subsequence of $\left\{v_{m}\right\}$ converges to $v$ with the desired properties as $m \rightarrow \infty$. For the rest of the proof of Proposition 9.1 see [AGHLV, section 11].

\section{The Hadamard variational formula for $\mathcal{A}$-Harmonic PDEs}

Let $E_{1}$ and $E_{2}$ be compact convex sets and suppose 0 is in the interior of $E_{1} \cap E_{2}$. Fix $p \geq n$ and let $u(\cdot, t)$ be the $\mathcal{A}=\nabla f$-harmonic Green's function for $\mathbb{R}^{n} \backslash\left(E_{1}+t E_{2}\right)$ with pole at infinity when $t \geq 0$. Also let $\mu_{E_{1}+t E_{2}}$ be the measure defined on $\mathbb{S}^{n-1}$ in (9.1) relative to $u(\cdot, t)$. In this section we prove

Proposition 10.1. With the above notation let $h_{1}$ and $h_{2}$ be the support functions for $E_{1}$ and $E_{2}$, respectively and let $\mathbf{g}\left(\cdot, E_{1}+t E_{2}\right)$ be the Gauss map for $\partial\left(E_{1}+t E_{2}\right)$. Then for $t \geq 0$ and $p=n$ ( $\gamma$ is as in Definition 5.4) we have

$\frac{d \mathcal{C}_{\mathcal{A}}\left(E_{1}+t E_{2}\right)}{d t}=n \gamma^{-1} \mathcal{C}_{\mathcal{A}}\left(E_{1}+t E_{2}\right) \int_{\partial\left(E_{1}+t E_{2}\right)} h_{2}\left(\mathbf{g}\left(x, E_{1}+t E_{2}\right)\right) f(\nabla u(x, t)) d \mathcal{H}^{n-1}$.

While for $n<p$ we have

$\frac{d \mathcal{C}_{\mathcal{A}}\left(E_{1}+t E_{2}\right)}{d t}=p(p-1) \mathcal{C}_{\mathcal{A}}\left(E_{1}+t E_{2}\right)^{\frac{p-2}{p-1}} \int_{\partial\left(E_{1}+t E_{2}\right)} h_{2}\left(\mathbf{g}\left(x, E_{1}+t E_{2}\right)\right) f(\nabla u(x, t)) d \mathcal{H}^{n-1}$.

Proof. To lay the groundwork for the proof of Proposition 10.1 we first argue as in (12.2)-(12.15) of [AGHLV]. We begin by assuming for $i=1,2$ that

$\partial E_{i}$ is locally the graph of an infinitely differentiable

and strictly convex function on $\mathbb{R}^{n-1}$.

We note from Lemma 3.4 that $u\left(\cdot, t_{i}\right)$, for $i=1,2$, has Hölder continuous second partials in $\mathbb{R}^{n} \backslash\left(E_{1}+t_{2} E_{2}\right)$. Let

$$
\zeta\left(x, t_{1}\right)=\frac{u\left(x, t_{1}\right)-u\left(x, t_{2}\right)}{t_{2}-t_{1}} \quad \text { whenever } x \in \mathbb{R}^{n} \text { and } 0<t_{1}<t_{2} .
$$


Then from Lemmas 5.2, 5.3, and the maximum principle for $\mathcal{A}$-harmonic functions we see that $\zeta \geq 0$. Moreover, from Lemma 6.1 we find as in (4.54)-(4.56) that $\zeta$ is a weak solution to

$$
\sum_{i, j=1}^{n} \frac{\partial}{\partial x_{i}}\left(\bar{d}_{i j} \zeta_{x_{j}}\right)=0
$$

in $\mathbb{R}^{n} \backslash\left(E_{1}+t_{2} E_{2}\right)$ where

$$
\bar{d}_{i j}(x)=\int_{0}^{1} f_{\eta_{i} \eta_{j}}\left(s \nabla u\left(x, t_{1}\right)+(1-s) \nabla u\left(x, t_{2}\right)\right) d s .
$$

Also,

$$
c^{-1} \bar{\sigma}(x)|\xi|^{2} \leq \sum_{i, j=1}^{n} \bar{d}_{i j}(x) \xi_{i} \xi_{j} \leq c \bar{\sigma}(x)|\xi|^{2}
$$

whenever $\xi \in \mathbb{R}^{n} \backslash\{0\}$ and $x \in \mathbb{R}^{n} \backslash\left(E_{1}+t_{2} E_{2}\right)$ with

$$
\bar{\sigma}(x) \approx\left(\left|\nabla u\left(x, t_{2}\right)\right|+\left|\nabla u\left(x, t_{1}\right)\right|\right)^{p-2} .
$$

The constants in (10.5) and (10.6) may depend on $t_{2}$ and the radius of the largest ball contained in $E_{2}$ but are independent of $x$ as above and $t_{1}$ whenever $t_{1} \in\left[t_{2} / 2, t_{2}\right)$. Also from Lemma 3.4, (8.6), Lemma 6.1 and the theorem in [Li, Theorem 1] mentioned earlier, we see that $\nabla u\left(\cdot, t_{i}\right)$ for $i=1,2$, extend to Hölder continuous functions in the closure of $\mathbb{R}^{n} \backslash\left(E_{1}+t_{i} E_{2}\right)$. More specifically, if

$$
t_{2} / 2 \leq t_{1}<t_{2} \quad \text { and } \quad \rho=4\left(t_{2}+1\right)\left(\operatorname{diam}\left(E_{1}\right)+\operatorname{diam}\left(E_{2}\right)\right)
$$

then there exist $\beta \in(0,1)$ and $C^{\star} \geq 1$, independent of $t_{1}$, such that for $i=1,2$,

$$
\begin{aligned}
& \text { (a) }\left|\nabla u\left(x, t_{i}\right)-\nabla u\left(y, t_{i}\right)\right| \leq C^{\star}|x-y|^{\beta}, \\
& \text { (b) }\left(C^{\star}\right)^{-1} \leq\left|\nabla u\left(x, t_{i}\right)\right| \leq C^{\star}
\end{aligned}
$$

whenever $x, y$ are in the closure of $B(0, \rho) \backslash\left(E_{1}+t_{i} E_{2}\right)$. We point out that the lower bound in (10.7) (b) follows from a contradiction type argument using Ascoli's theorem and Lemma 6.1. From (10.7) (b) and the mean value theorem from calculus we see that there exists $C^{\star \star}$ independent of $t_{1} \in\left[t_{2} / 2, t_{2}\right)$ and $x$ such that

$$
0 \leq \zeta \leq C^{\star \star}
$$

on $\partial\left(E_{1}+t_{2} E_{2}\right)$. From (10.8), Lemmas 5.2, 5.3, and the maximum principle for $\mathcal{A}$ harmonic functions, we deduce for given $\epsilon>0$ that there exists $s=s(\epsilon)>\rho$ such that

$$
\zeta \leq C^{* *}+\epsilon u\left(\cdot, t_{1}\right) \text { in } B(0, s(\epsilon)) \backslash\left(E_{1}+t_{2} E_{2}\right) .
$$

Letting $\epsilon \rightarrow 0$ it follows that (10.8) is valid in the closure of $\mathbb{R}^{n} \backslash\left(E_{1}+t_{2} E_{2}\right)$. In a similar way we get that $M(\cdot, \zeta)$ is decreasing and $m(\cdot, \zeta)$ is increasing on $(\rho, \infty)$. Using 
this fact and arguing as in (6.1) (b), we find that if $u\left(\cdot, t_{i}\right)=F+k_{i}$, for $i=1,2$, then there exists $\hat{\theta}>0$ such that

$$
|\zeta(x)-\zeta(\infty)|=\left|\zeta(x)-\frac{k_{1}(\infty)-k_{2}(\infty)}{t_{2}-t_{1}}\right| \leq 2 C^{\star \star}\left(\frac{\rho}{|x|}\right)^{\hat{\theta}} \text { for }|x| \geq \rho .
$$

In this discussion, $F$ is the Fundamental solution as in Lemma 4.4 when $p=n$ and as in Lemma 4.6 when $n<p<\infty$. Also, $k_{i}$ is the function associated to $E_{1}+t_{i} E_{2}$ for $i=1,2$ as in Lemma 5.2 when $p=n$ and as in Lemma 5.3 when $n<p<\infty$. Moreover, the constant $\hat{\theta}$ is independent of $t_{1} \in\left[t_{2} / 2, t_{2}\right)$ and $x$ as above.

From (3.3) and Lemmas 6.1 we see that $\bar{d}_{i j}(x)$ in (10.4) are Lipschitz continuous in $\mathbb{R}^{n} \backslash B(0, \rho)$ with Lipschitz norm independent of $t_{1} \in\left[t_{2} / 2, t_{2}\right)$. Using this fact, elliptic PDE theory (see $[\mathrm{GT}]$ ) and (10.9) we see that $\nabla \zeta$ is locally Hölder continuous in $\mathbb{R}^{n} \backslash B(0, \rho)$ and

$$
|\nabla \zeta(x)| \leq \tilde{C}|x|^{-1-\hat{\theta}} \quad \text { whenever }|x| \geq \rho
$$

where $\tilde{C}$ has the same dependence as $C^{\star \star}$. From (10.7) (a), Lemma 6.1, and uniqueness of $u\left(\cdot, t_{i}\right)$ for $i=1,2$, we deduce that

$\nabla u\left(\cdot, t_{1}\right) \rightarrow \nabla u\left(\cdot, t_{2}\right)$ uniformly on the closure of $\mathbb{R}^{n} \backslash\left(E_{1}+t_{2} E_{2}\right)$ as $t_{1} \rightarrow t_{2}$.

From (10.8)-(10.11), Lemma 6.1, (10.4)-(10.6), and Caccioppoli type estimates for locally uniformly elliptic PDE, we deduce that if $t_{1} \rightarrow t_{2}$ through an increasing sequence, say $\left\{s_{m}\right\}$, then a subsequence of $\left\{\zeta=\zeta\left(\cdot, s_{m}\right)\right\}$, also denoted by $\left\{\zeta\left(\cdot, s_{m}\right)\right\}$, converges uniformly on compact subsets of $\left(\mathbb{R}^{n} \cup\{\infty\}\right) \backslash\left(E_{1}+t_{2} E_{2}\right)$ to a locally Hölder $\hat{\beta}$ continuous function, say $\breve{\zeta}$. Moreover, this subsequence also converges to $\breve{\zeta}$ locally weakly in $W^{1,2}$ of $\mathbb{R}^{n} \backslash\left(E_{1}+t_{2} E_{2}\right)$. Finally,

$$
\sum_{i, j=1}^{n} \frac{\partial}{\partial x_{i}}\left(f_{\eta_{i} \eta_{j}}\left(\nabla u\left(x, t_{2}\right)\right) \breve{\zeta}_{x_{j}}(x)\right)=0
$$

locally in the weak sense in $\mathbb{R}^{n} \backslash\left(E_{1}+t_{2} E_{2}\right)$ and (10.9), (10.10) are valid with $\zeta$ replaced by $\breve{\zeta}$.

Next we show that $\breve{\zeta}$ has boundary values that are independent of the choice of sequence. To do this, for $k=1,2$ we let $x_{k}(Z)=\nabla h_{k}(Z)$ whenever $Z \in \mathbb{S}^{n-1}$ and recall that $x_{k}(Z) \in \partial E_{k}$ with $Z=\mathbf{g}\left(x_{k}(Z), E_{k}\right)$ for $k=1,2$. We fix $X, Y \in \mathbb{S}^{n-1}$, write $x, y$ for $x_{1}(X)+t_{2} x_{2}(X), x_{1}(Y)+t_{2} x_{2}(Y)$ respectively and note that

$x=\mathbf{g}^{-1}\left(X, E_{1}+t_{2} E_{2}\right) \in \partial\left(E_{1}+t_{2} E_{2}\right) \quad$ and $\quad y=\mathbf{g}^{-1}\left(Y, E_{1}+t_{2} E_{2}\right) \in \partial\left(E_{1}+t_{2} E_{2}\right)$.

We consider two cases. First if

$$
|x-y| \leq d\left(x, E_{1}+t_{1} E_{2}\right) / 2
$$

then from (10.7) (a) and the mean value theorem of calculus we have

$$
|\zeta(x)-\zeta(y)-\langle\nabla \zeta(x), x-y\rangle| \leq \hat{C}|x-y|^{\beta}
$$


where $\hat{C}$ is independent of $t_{1}$. Second, if

$$
|x-y|>d\left(x, E_{1}+t_{1} E_{2}\right) / 2
$$

then using $u\left(\cdot, t_{1}\right) \equiv 0$ on $\partial\left(E_{1}+t_{1} E_{2}\right)$ and the same strategy as above we see that

$$
\begin{aligned}
& \mid \zeta(x)-\left\langle\nabla u \left( x_{1}(X)+\right.\right.\left.\left.t_{1} x_{2}(X), t_{1}\right), x_{2}(X)\right\rangle \mid \\
&+\mid \zeta(y)-\left\langle\nabla u\left(x_{1}(Y)+t_{1} x_{2}(Y), t_{1}\right), y_{2}(Y)\right\rangle \mid \\
& \leq \hat{C}|x-y|^{\beta} .
\end{aligned}
$$

Now $h_{1}+t_{1} h_{2}$ is the support function for $E_{1}+t_{1} E_{2}$ and so

$$
\begin{aligned}
\left\langle\nabla u\left(x_{1}(X)+t_{1}\left(x_{2}(X)\right), t_{1}\right), x_{2}(X)\right\rangle & =\left|\nabla u\left(x_{1}(X)+t_{1}\left(x_{2}(X)\right), t_{1}\right)\right|\left\langle X, x_{2}(X)\right\rangle \\
& =\left|\nabla u\left(x, t_{1}\right)\right| h_{2}\left(\mathbf{g}\left(x, E_{1}+t_{2} E_{2}\right)\right)+\lambda(x) \\
& =I I I_{1}+\lambda(x)
\end{aligned}
$$

where $|\lambda(x)| \leq \bar{C}|x-y|^{\beta}$ and $\bar{C}$ is independent of $t_{1}$. Similarly,

$$
\begin{aligned}
\left\langle\nabla u\left(x_{1}(Y)+t_{1} x_{2}(Y), t_{1}\right), x_{2}(Y)\right\rangle & =\left|\nabla u\left(y, t_{1}\right)\right| h_{2}\left(\mathbf{g}\left(y, E_{1}+t_{2} E_{2}\right)\right)+\bar{\lambda}(y) \\
& =I I I_{2}+\bar{\lambda}(y)
\end{aligned}
$$

where $\bar{\lambda}(y)$ satisfies the same inequality as $\lambda(x)$. From (10.14)-(10.16) and the triangle inequality we find that

$$
|\zeta(x)-\zeta(y)| \leq\left|I I I_{1}-I I I_{2}\right|+|\lambda(x)|+|\bar{\lambda}(y)| \leq \tilde{C}|x-y|^{\beta}
$$

where $\tilde{C}$ is independent of $t_{1} \in\left[t_{2} / 2, t_{2}\right)$. Here we have also used Lipschitzness of $h_{2}$ and $(10.7)(a)$ to estimate $\left|I I I_{1}-I I I_{2}\right|$. From (10.17), we deduce that $\zeta=\zeta\left(\cdot, t_{1}\right)$ is Hölder $\beta$-continuous on $\partial\left(E_{1}+t_{2} E_{2}\right)$ with Hölder norm bounded by a constant independent of $t_{1} \in\left[t_{2} / 2, t_{2}\right)$. From well-known theorems for divergence form uniformly elliptic PDE with bounded measurable coefficients it now follows that $\zeta$ is Hölder $\tau$ continuous on the closure of $B(0, \rho) \backslash\left(E_{1}+t_{2} E_{2}\right)$ for some $\tau>0$ with Hölder norm independent of $t_{1} \in\left[t_{2} / 2, t_{2}\right)$ This fact and the same reasoning as in (10.10) yield

$$
|\nabla \zeta(x)| \leq C d\left(x, \partial\left(E_{1}+t_{2} E_{2}\right)\right)^{-1+\tau} .
$$

Taking limits we conclude from Ascoli's theorem that $\breve{\zeta}$ is the uniform limit of $\left(\zeta\left(\cdot, s_{m}\right)\right)$ in the closure of $B(0, \rho) \backslash\left(E_{1}+t_{2} E_{2}\right)$. Thus $\breve{\zeta}$ is also Hölder $\tau$-continuous in the closure of $B(0, \rho) \backslash\left(E_{1}+t_{2} E_{2}\right)$ and (10.18) holds for $\breve{\zeta}$. Finally, using (10.7) and arguing as in (10.15) we see that

$$
\left|\zeta\left(x, t_{1}\right)-\right| \nabla u\left(x, t_{2}\right)\left|h_{2}\left(\mathbf{g}\left(x, E_{1}+t_{2} E_{2}\right)\right)\right| \leq c\left|t_{1}-t_{2}\right|^{\beta} .
$$

From this estimate we conclude that

$$
\zeta\left(x, t_{1}\right) \rightarrow\left|\nabla u\left(x, t_{2}\right)\right| h_{2}\left(\mathbf{g}\left(x, E_{1}+t_{2} E_{2}\right)\right) \quad \text { as } \quad t_{1} \rightarrow t_{2}
$$


whenever $x \in \partial\left(E_{1}+t_{2} E_{2}\right)$. From (10.19) we see that every convergent subsequence of $\left\{\zeta\left(\cdot, t_{1}\right)\right\}$ converges to a weak solution of (10.12) satisfying (10.18) with continuous boundary values

$$
\left|\nabla u\left(\cdot, t_{2}\right)\right| h_{2}\left(\mathbf{g}\left(\cdot, E_{1}+t_{2} E_{2}\right)\right) \quad \text { on } \quad \partial\left(E_{1}+t_{2} E_{2}\right) .
$$

To begin the proof of Proposition 10.1 in the smooth case and when $t_{2} / 2 \leq t_{1}<t_{2}$ recall from the display above (10.7), that $E_{1}+t_{2} E_{2} \subset B(0, \rho)$. If $R>4 \rho$ we apply the divergence theorem to $u\left(\cdot, t_{i}\right)(\nabla f)\left(\nabla u\left(\cdot, t_{i}\right)\right.$ for $i=1,2$ and use $\mathcal{A}$-harmonicity of $u\left(\cdot, t_{i}\right), p$-homogeneity of $f$, and smoothness of $u\left(\cdot, t_{i}\right)$ on $\partial\left(E_{1}+t_{i} E_{2}\right), i=1,2$ to get

$$
\begin{aligned}
I_{i}(R) & =p \int_{B(0, R) \backslash\left(E_{1}+t_{i} E_{2}\right)} f\left(\nabla u\left(x, t_{i}\right)\right) d x \\
& =\int_{\partial B(0, R)} u\left(x, t_{i}\right)\left\langle(\nabla f)\left(\nabla u\left(x, t_{i}\right)\right), x /|x|\right\rangle d \mathcal{H}^{n-1} .
\end{aligned}
$$

For brevity we write for fixed $t_{2}$,

$$
\frac{I_{1}(R)-I_{2}(R)}{t_{2}-t_{1}}=\int_{\partial B(0, R)} J\left(\cdot, t_{1}\right) d \mathcal{H}^{n-1} .
$$

To make calculations first observe that

$$
\left\langle\frac{(\nabla f)\left(\nabla u\left(x, t_{1}\right)\right)-(\nabla f)\left(\nabla u\left(x, t_{2}\right)\right)}{t_{2}-t_{1}}, x /|x|\right\rangle=\sum_{l, j=1}^{n} \bar{d}_{l j}(x) \zeta_{x_{j}}(x)\left(x_{l} /|x|\right)
$$

where $\left(\bar{d}_{l j}\right), 1 \leq l, j \leq n$, is as in (10.4). Letting $t_{1} \rightarrow t_{2}$ in $\left(s_{m}\right)$ we see that

$$
\left\langle\frac{(\nabla f)\left(\nabla u\left(x, t_{1}\right)\right)-(\nabla f)\left(\nabla u\left(x, t_{2}\right)\right)}{t_{2}-t_{1}}, x /|x|\right\rangle \rightarrow \sum_{l, j=1}^{n} \bar{a}_{l j}(x) \breve{\zeta}_{x_{j}}(x)\left(x_{l} /|x|\right)
$$

where $\bar{a}_{l j}(x)=\frac{\partial^{2} f}{\partial \eta_{l} \partial \eta_{j}}\left(\nabla u\left(x, t_{2}\right)\right)$. Next we use the same algebra as in the calculus argument for finding the derivative of a product. After that we note from (10.21) and (10.10) that

$$
J\left(x, t_{1}\right) \rightarrow J_{1}(x)=\breve{\zeta}(x)\left\langle(\nabla f)\left(\nabla u\left(x, t_{2}\right)\right), x /|x|\right\rangle+u\left(x, t_{2}\right) \sum_{l, j=1}^{n} \bar{a}_{l j}(x) \breve{\zeta}_{x_{j}}(x) x_{l} /|x|
$$

as $t_{1} \rightarrow t_{2}$ in $\left(s_{m}\right)$.

Using (6.1) $(a)$ and $(b)$ with $k_{2}=k$ and arguing in a now well known way we deduce that $k_{2}$ is a solution in $B(x,|x| / 2)$ to a uniformly elliptic PDE in divergence form with Lipschitz continuous coefficients when $|x|>4 R_{0}$. From (6.1)(b) and elliptic PDE theory it follows that

$$
\left|\nabla k_{2}(x)\right| \leq C|x|^{-1-\tilde{\theta}} \quad \text { for }|x|=R
$$


where once again $C$ is independent of $x$. Using (10.23), (2.6) (i), and our knowledge of $F$ it follows that

$$
u\left(x, t_{2}\right) \bar{a}_{l j}(x)=F(x) \frac{\partial^{2} f}{\partial \eta_{l} \partial \eta_{j}}(\nabla F(x))+o\left(\delta(R) R^{\frac{(1-n)(p-2)+(p-n)}{p-1}}\right) \quad \text { as } R \rightarrow \infty
$$

where the $o$ term is independent of $t_{1} \in\left[t_{2} / 2, t_{2}\right)$. Here $\delta(R)=1$ if $p>n$ and $\delta(R)=\log R$, for $p=n$. Likewise, as $R \rightarrow \infty$,

$$
\left\langle(\nabla f)\left(\nabla u\left(x, t_{2}\right)\right), x /|x|\right\rangle=\langle(\nabla f)(\nabla F(x)), x /|x|\rangle+o\left(R^{1-n}\right) .
$$

Using (10.10), (10.24), (10.25), and $\breve{\zeta}(x)=\breve{\zeta}(\infty)+o(1)$ as $R \rightarrow \infty$ in (10.22) we conclude first that

$$
J_{1}(x)=\breve{\zeta}(\infty)\langle(\nabla f)(\nabla F(x)), x /|x|\rangle+o\left(R^{1-n}\right)
$$

and second since $F$ is a fundamental solution that

$$
\begin{aligned}
\lim _{m \rightarrow \infty} \frac{I_{1}(R)-I_{2}(R)}{t_{2}-t_{1}} & =\breve{\zeta}(\infty) \int_{\partial B(0, R)}\langle(\nabla f)(\nabla F(x)), x /|x|\rangle d \mathcal{H}^{n-1}+o(1) \\
& =\breve{\zeta}(\infty)+o(1) .
\end{aligned}
$$

Next we write

$$
\frac{I_{1}(R)-I_{2}(R)}{t_{2}-t_{1}}=T_{1}(R)+T_{2}(R)
$$

where

$$
T_{1}(R):=p\left(t_{2}-t_{1}\right)^{-1} \int_{B(0, R) \backslash\left(E_{1}+t_{2} E_{2}\right)}\left(f\left(\nabla u\left(x, t_{1}\right)\right)-f\left(\nabla u\left(x, t_{2}\right)\right)\right) d x
$$

and

$$
T_{2}(R):=p\left(t_{2}-t_{1}\right)^{-1} \int_{\left(E_{1}+t_{2} E_{2}\right) \backslash\left(E_{1}+t_{1} E_{2}\right)}\left(f\left(\nabla u\left(x, t_{1}\right)\right) d x .\right.
$$

From (10.11) and (10.19) we have

$$
\begin{aligned}
T_{2}(R) & =\left(t_{2}-t_{1}\right)^{-1} \int_{\left(E_{1}+t_{2} E_{2}\right) \backslash\left(E_{1}+t_{1} E_{2}\right)} \nabla \cdot\left[\left(u\left(x, t_{1}\right)\right) \nabla f\left(\nabla u\left(x, t_{1}\right)\right)\right] d x \\
& =\int_{\partial\left(E_{1}+t_{2} E_{2}\right)} \zeta(x)\left|\nabla u\left(x, t_{2}\right)\right|^{-1}\left\langle\nabla f\left(\nabla u\left(x, t_{1}\right)\right), \nabla u\left(x, t_{2}\right)\right\rangle d \mathcal{H}^{n-1} \\
& \rightarrow \hat{T}_{2}:=p \int_{\partial\left(E_{1}+t_{2} E_{2}\right)} h_{2}\left(\mathbf{g}\left(x, E_{1}+t_{2} E_{2}\right)\right) f\left(\nabla u\left(x, t_{2}\right)\right) d \mathcal{H}^{n-1} \quad \text { as } t_{1} \rightarrow t_{2} \text { in }\left(s_{m}\right) .
\end{aligned}
$$

To handle $T_{1}(R)$ observe as in (10.21) that

$$
p \frac{f\left(\nabla u\left(x, t_{1}\right)\right)-f\left(\nabla u\left(x, t_{2}\right)\right)}{t_{2}-t_{1}}=\sum_{j=1}^{n} q_{j}(x) \zeta_{x_{j}}(x)
$$


where

$$
q_{j}(x)=p \int_{0}^{1} \frac{\partial f}{\partial \eta_{j}}\left(s \nabla u\left(x, t_{1}\right)+(1-s) \nabla u\left(x, t_{2}\right)\right) d s .
$$

Using (10.30) in the definition of $T_{1}(R)$ and letting $t_{1} \rightarrow t_{2}$ in $\left(s_{m}\right)$ we obtain from (10.18) and the Lebesgue dominated convergence theorem that

$$
\begin{aligned}
T_{1}(R) \rightarrow \hat{T}_{1}(R) & =p \sum_{j=1}^{n} \int_{B(0, R) \backslash\left(E_{1}+t_{2} E_{2}\right)} \frac{\partial f}{\partial \eta_{j}}\left(\nabla u\left(x, t_{2}\right)\right) \breve{\zeta}_{x_{j}}(x) d x \\
& =\frac{p}{p-1} \sum_{l, j=1}^{n} \int_{B(0, R) \backslash\left(E_{1}+t_{2} E_{2}\right)} \bar{a}_{l j}(x) u_{x_{l}}\left(x, t_{2}\right) \breve{\zeta}_{x_{j}}(x) d x
\end{aligned}
$$

where $\bar{a}_{l j}(x)=\frac{\partial^{2} f}{\partial \eta_{l} \partial \eta_{j}}\left(\nabla u\left(x, t_{2}\right)\right)$ as earlier and we have used the $(p-1)$-homogeneity of $\nabla f$. From (10.18) we see that the above integral can be integrated by parts to obtain,

$$
\hat{T}_{1}(R)=\frac{p}{p-1} \sum \int_{\partial B(0, R)} u\left(x, t_{2}\right) \sum_{l, j=1}^{n} \bar{a}_{l j}(x) \breve{\zeta}_{x_{j}}(x) x_{l} /|x| d \mathcal{H}^{n-1}
$$

where we have also used (10.12). Letting $R \rightarrow \infty$ we deduce as in the derivation of (10.27) that $\lim _{R \rightarrow \infty} \hat{T}_{1}(R)=0$. Combining this equality, (10.29) and letting $R \rightarrow \infty$ in $(10.27)$ we arrive at

$$
\breve{\zeta}(\infty)=p \int_{\partial\left(E_{1}+t_{2} E_{2}\right)} h_{2}\left(\mathbf{g}\left(x, E_{1}+t_{2} E_{2}\right)\right) f\left(\nabla u\left(x, t_{2}\right)\right) d \mathcal{H}^{n-1} .
$$

We note that (10.31), the remark after (10.19), and the usual maximum principle argument imply that $\breve{\zeta}$ is independent of the choice of $\left(s_{m}\right)$. Thus we put

$$
\breve{\zeta}(\infty)=\lim _{t_{1} \rightarrow t_{2}^{-}} \zeta\left(\infty, t_{1}\right)=-\frac{d k}{d t_{2}}(\infty)
$$

where $-k(\cdot, t)=F(\cdot)-u(\cdot, t)$ for $t \in[0, \infty)$. Now from Theorem $\mathrm{A}$ we see that $t \mapsto \mathcal{C}_{\mathcal{A}}\left(E_{1}+t E_{2}\right)$ is concave on $[0, \infty)$ when $p=n$ and $t \mapsto \mathcal{C}_{\mathcal{A}}^{\frac{1}{p-n}}\left(E_{1}+t E_{2}\right)$ is concave on $[0, \infty)$ when $p>n$. Thus $t \mapsto \mathcal{C}_{\mathcal{A}}\left(E_{1}+t E_{2}\right)$ is Lipschitz and differentiable off a countable set when $n \leq p<\infty$. From this observation, (10.31), and the chain rule we have when $p=n$

$$
\begin{aligned}
& \frac{d \mathcal{C}_{\mathcal{A}}\left(E_{1}+t E_{2}\right)}{d t}=-\gamma^{-1} \mathcal{C}_{\mathcal{A}}\left(E_{1}+t E_{2}\right) \frac{d k}{d t}(\infty) \\
& \quad=n \gamma^{-1} \mathcal{C}_{\mathcal{A}}\left(E_{1}+t E_{2}\right) \int_{\partial\left(E_{1}+t E_{2}\right)} h_{2}\left(\mathbf{g}\left(x, E_{1}+t E_{2}\right)\right) f(\nabla u(x, t)) d \mathcal{H}^{n-1}
\end{aligned}
$$


and for $p>n$

$$
\begin{aligned}
& \frac{d \mathcal{C}_{\mathcal{A}}\left(E_{1}+t E_{2}\right)}{d t}=-(p-1) \mathcal{C}_{\mathcal{A}}\left(E_{1}+t E_{2}\right)^{\frac{p-2}{p-1}} \frac{d k}{d t}(\infty) \\
& =p(p-1) \mathcal{C}_{\mathcal{A}}\left(E_{1}+t E_{2}\right)^{\frac{p-2}{p-1}} \int_{\partial\left(E_{1}+t E_{2}\right)} h_{2}\left(\mathbf{g}\left(x, E_{1}+t E_{2}\right)\right) f(\nabla u(x, t)) d \mathcal{H}^{n-1}
\end{aligned}
$$

except for at most $t$ in a countable set. Now from properties of support functions and (10.11) we see that the right-hand side in both equalities is continuous as a function of $t$ so from the usual calculus argument $\frac{d \mathcal{C}_{\mathcal{A}}\left(E_{1}+t E_{2}\right)}{d t}$ exists on $(0, \infty)$ and has a left-hand derivative at $t=0$. Thus Proposition 10.1 is valid under assumption (10.3).

To show this assumption can be removed, choose sequences of uniformly bounded convex domains $\left\{E_{1}^{(l)}\right\}_{l \geq 1}$ and $\left\{E_{2}^{(l)}\right\}_{l \geq 1}$ with $E_{i} \subset E_{i}^{(l)}$ for $i=1,2$ and $l=1,2, \ldots$, satisfying (10.3) with $\partial E_{i}$ replaced by $\partial E_{i}^{(l)}, i=1,2$ and $l=1,2, \ldots$ We also choose these sequences so that $E_{i}^{(l)}$ converges to $E_{i}$ in the sense of Hausdorff distance as $l \rightarrow \infty$. Let $u^{(l)}(\cdot, t)$ be the $\mathcal{A}$-harmonic Green's function for the complement of $E_{1}^{(l)}+t E_{2}^{(l)}$ with pole at $\infty$. We set $-k^{(l)}(\cdot, t)=F(\cdot)-u^{(l)}(\cdot, t)$ while as earlier $u(\cdot, t)$ and $k(\cdot, t)$ have the same meaning relative to $E_{1}+t E_{2}$. We claim that

$$
\lim _{l \rightarrow \infty} k^{(l)}(\infty, t)=k(\infty, t) \quad \text { for } t \in[0, \infty) .
$$

This claim will be proved in more generality in (11.1) so we reserve its proof until the next section. Let

$$
\Phi(t)=\int_{\partial\left(E_{1}+t E_{2}\right)} h_{2}\left(\mathbf{g}\left(x, E_{1}+t E_{2}\right)\right) f(\nabla u(x, t)) d \mathcal{H}^{n-1}
$$

and let $\Phi_{l}(t)$ denote the function in this display with $E_{i}$, replaced by $E_{i}^{(l)}, i=1,2, l=$ $1,2, \ldots$ Given $0<a<\infty$ we claim there exist $m=m(a)$ and $M=M(a)$ such that for $l \geq m$, we have

$$
0<\Phi_{l}(t) \leq M \quad \text { for } \quad t \in[0, a]
$$

To verify this assertion fix $l, t$, let

$$
E_{0}=E_{1}^{(l)}+t E_{2}^{(l)}
$$

and let $h_{0}$ be the support function for $E_{0}$ and let $\mathbf{g}\left(\cdot, E_{0}\right)$ be the Gauss map for $\partial E_{0}$ while $u_{0}$ is the $\mathcal{A}=\nabla f$-harmonic Green's function for $\mathbb{R}^{n} \backslash E_{0}$ with pole at infinity. Applying Proposition 10.1 in this case with $E_{1}, E_{2}, t$, replaced by $E_{0}, E_{0}, 0$, and using the fact that

$$
\mathcal{C}_{\mathcal{A}}\left((1+t) E_{0}\right)= \begin{cases}(1+t) \mathcal{C}_{\mathcal{A}}\left(E_{0}\right) & \text { when } p=n, \\ (1+t)^{p-n} \mathcal{C}_{\mathcal{A}}\left(E_{0}\right) & \text { when } n<p<\infty\end{cases}
$$

we obtain when $p=n$

$$
\gamma=n \int_{\partial E_{0}} h_{0}\left(\mathbf{g}\left(x, E_{0}\right)\right) f\left(\nabla u_{0}(x)\right) d \mathcal{H}^{n-1} .
$$


While when $p>n$ we have

$$
\frac{p-n}{p-1} \mathcal{C}_{\mathcal{A}}\left(E_{0}\right)^{1 /(p-1)}=p \int_{\partial E_{0}} h_{0}\left(\mathbf{g}\left(x, E_{0}\right)\right) f\left(\nabla u_{0}(x)\right) d \mathcal{H}^{n-1} .
$$

Since $E_{0}$ is uniformly bounded and $h_{0} \geq \min _{\mathbb{S}^{n-1}} h_{1}>0$, where $h_{1}$ is the support function for $E_{1}$, it follows from (10.36), (10.37), and properties of $\mathcal{C}_{\mathcal{A}}(\cdot)$ that $(10.35)$ is true. From (10.34), (10.35), Proposition 9.1, Proposition 10.1 in the smooth case, and Lipschitzness of support functions, as well as the Lebesgue dominated convergence theorem, we find that

$$
\begin{aligned}
k(\infty, t)-k(\infty, 0) & =\lim _{l \rightarrow \infty}\left[k^{(l)}(\infty, t)-k^{(l)}(\infty, 0)\right] \\
& =-\lim _{l \rightarrow \infty} p \int_{0}^{t} \Phi_{l}(s) d s=-p \int_{0}^{t} \Phi(s) d s .
\end{aligned}
$$

Also $\Phi$ is continuous on $[0, \infty)$ by Proposition 9.1. Now $(10.38)$, the definition of $\mathcal{C}_{\mathcal{A}}(\cdot)$, and the Lebesgue differentiation theorem yield Proposition 10.1 without assumption (10.3).

Remark 10.2. We note that Proposition 10.1 remains valid for $t>0$ if we assume only that $0 \in E_{1}$, rather than 0 is in the interior of $E_{1}$ (so $\mathcal{H}^{n}\left(E_{1}\right)=0$ is possible but from the definition of $E_{2}$ we still have 0 in the interior of $E_{2}$ ). To handle this case we put $E_{1}^{\prime}=E_{1}+t E_{2}$ and $E_{2}^{\prime}=E_{2}$. Then $E_{1}^{\prime}, E_{2}^{\prime}$ are compact convex sets and 0 is in the interior of $E_{1}^{\prime} \cap E_{2}^{\prime}$. Applying Proposition 10.1 with $E_{1}, E_{2}$ replaced by $E_{1}^{\prime}, E_{2}^{\prime}$ respectively and at $t=0$ we obtain the above generalization of Proposition 10.1.

\section{Proof of Theorem B}

Finally we are in a position to prove existence of the measure in Theorem B in the discrete case. Once again our argument is similar to the one in [AGHLV, section 13] for $1<p<n$. However, in our opinion not all of this argument is so well known and involves different calculations for $p \geq n$, so we will give mostly full details. To begin we note that if $p \geq n$ is fixed, $E^{\prime}$ is a compact convex set and $\left(E_{l}^{\prime}\right)$ is a sequence of convex compact sets converging to $E^{\prime}$ in the sense of Hausdorff distance, then either $E^{\prime}$ is a single point in which case $\lim _{l \rightarrow \infty} \mathcal{C}_{\mathcal{A}}\left(E_{l}^{\prime}\right)=0$ or

$$
\lim _{l \rightarrow \infty} \mathcal{C}_{\mathcal{A}}\left(E_{l}^{\prime}\right)=\mathcal{C}_{\mathcal{A}}\left(E^{\prime}\right)>0
$$

Note that (11.1) and the definition of $\mathcal{C}_{\mathcal{A}}(\cdot)$ give claim (10.34). If $E^{\prime}$ is a single point it follows from (5.23) that the above limit is zero. Otherwise, let $G_{l}^{\prime}, l=1,2, \ldots$, be the corresponding sequence of $\mathcal{A}$-harmonic Green's functions for $\mathbb{R}^{n} \backslash E_{l}^{\prime}$ for $l=1,2, \ldots$, and let $G^{\prime}$ be the $\mathcal{A}$-harmonic Green's functions for $\mathbb{R}^{n} \backslash E^{\prime}$ with pole at infinity. Then from Lemma 3.5 and uniqueness of $G^{\prime}$ we see that $\left(G_{l}^{\prime}\right)$ converges uniformly on compact subsets of $\mathbb{R}^{n}$ to $G^{\prime}$. From Lemma 6.1 we see that $\Theta=\left\{x: G^{\prime}(x) \leq 1\right\}$ is convex and has nonempty interior. Also $G^{\prime}-1$ is the $\mathcal{A}$-harmonic Green's function for $\mathbb{R}^{n} \backslash \Theta$ with pole at infinity. Translating and dilating $\Theta$ if necessary we may assume 
that $0 \in \Theta$ and $\operatorname{diam}(\Theta)=1$ thanks to (5.23). Applying (6.1) (a) to $G^{\prime}-1$ it follows as in the proof of $(6.1)(b)$, that there exists $R>0$ independent of $l$ so that

$$
\left|G_{l}^{\prime}-G^{\prime}\right|(x) \leq M\left(R,\left|G_{l}^{\prime}-G^{\prime}\right|\right) \quad \text { and } \quad 0<\min _{\partial B(0, R)}\left(F-G^{\prime}\right) \leq\left(F-G^{\prime}\right)(x)
$$

when $x \in \mathbb{R}^{n} \backslash B(0, R)$. Using these inequalities and the definition of $\mathcal{C}_{\mathcal{A}}(\cdot)$ in Definition 5.4 we obtain (11.1).

11.1. Proof of existence in Theorem B in the discrete case. Let $c_{1}, c_{2}, \ldots, c_{m}$ be positive numbers and $\xi_{i} \in \mathbb{S}^{n-1}$ for $1 \leq i \leq m$. Assume that $\xi_{i} \neq \xi_{j}$ for $i \neq j$ and let $\delta_{\xi_{i}}$ denote the measure with a point mass at $\xi_{i}$. Let $\mu$ be a measure on $\mathbb{S}^{n-1}$ with

$$
\mu(K)=\sum_{i=1}^{m} c_{i} \delta_{\xi_{i}}(K) \text { whenever } K \subset \mathbb{S}^{n-1} \text { is a Borel set. }
$$

We also assume that $\mu$ satisfies (7.1) $(i)$ and (ii). That is,

$$
\sum_{i=1}^{m} c_{i}\left|\left\langle\theta, \xi_{i}\right\rangle\right|>0 \quad \text { for all } \theta \in \mathbb{S}^{n-1}
$$

and

$$
\sum_{i=1}^{m} c_{i} \xi_{i}=0
$$

For technical reasons we also first assume that

$$
\text { either } \mu(\{\xi\}) \text { or } \mu(\{-\xi\})=0 \text { whenever } \xi \in \mathbb{S}^{n-1} .
$$

This assumption will be removed in the general proof of existence. For $\mu$ as above and fixed $p \geq n$, we show that there is a compact convex polyhedron $E$ with 0 in the interior of $E$ and

$$
\mu(K)=\int_{\mathbf{g}^{-1}(K, E)} f(\nabla U) d \mathcal{H}^{n-1} \quad \text { whenever } \quad K \subset \mathbb{S}^{n-1} \text { is a Borel set }
$$

where $\mathbf{g}(\cdot, E)$ is the Gauss map for $\partial E$ and $U$ is the $\mathcal{A}$-harmonic Green's function for $\mathbb{R}^{n} \backslash E$ with pole at infinity. Moreover, if $F_{i}$ denotes the face of $\partial E$ with outer normal $\xi_{i}$ for $1 \leq i \leq m$ then $\mathbf{g}\left(F_{i}, E\right)=\xi_{i}$ and

$$
\mu\left(\left\{\xi_{i}\right\}\right)=c_{i}=\int_{F_{i}} f(\nabla U) d \mathcal{H}^{n-1} \quad \text { for } 1 \leq i \leq m .
$$

Existence in (11.5) follows from a variational type argument apparently due to Minkowski (see $[\mathrm{G}$, Section 8.2])). Using this method one needs to show that a certain minimum problem has a solution within the given class of compact convex sets with nonempty interior. To be more specific let $q=\left(q_{1}, \ldots, q_{m}\right) \in \mathbb{R}^{m}$ with $q_{i} \geq 0$ for $1 \leq i \leq m$. Let

$$
E(q):=\bigcap_{i=1}^{m}\left\{x:\left\langle x, \xi_{i}\right\rangle \leq q_{i}\right\} \quad \text { and } \quad \Phi:=\left\{E(q): \mathcal{C}_{\mathcal{A}}(E(q)) \geq 1\right\}
$$


We also set

$$
\theta(q)=\sum_{i=1}^{m} c_{i} q_{i} \quad \text { and } \quad \lambda=\inf \{\theta(q): E(q) \in \Phi\} .
$$

We want to show there exists

$$
\breve{q}=\left(\breve{q}_{1}, \ldots, \breve{q}_{m}\right), \breve{q}_{i}>0 \text { for } 1 \leq i \leq m \text {, with } \theta(\breve{q})=\lambda \text { and } \mathcal{C}_{\mathcal{A}}(E(\breve{q})) \geq 1 \text {. }
$$

Using (11.6) and a variational type argument, it follows easily from Proposition 10.1 that $\mu$ is a constant multiple of the measure defined in (7.2) (c) relative to the $\mathcal{A}$ harmonic Green's function for $\mathbb{R}^{n} \backslash E(\breve{q})$ with a pole at infinity.

To begin the proof of (11.6) we first note that if $E(q) \in \Phi$ then $E(q)$ is a closed convex set. Also we note from (11.3) that

$$
\int_{\mathbb{S}^{n-1}}\langle\tau, \xi\rangle^{+} d \mu(\xi)=\int_{\mathbb{S}^{n-1}}\langle\tau, \xi\rangle^{-} d \mu(\xi) \quad \text { whenever } \quad \tau \in \mathbb{S}^{n-1}
$$

where $a^{+}=\max (a, 0)$ and $a^{-}=\max (-a, 0)$. From this note and (11.2) we see that for some $\phi>0$,

$$
\phi<\int_{\mathbb{S}^{n-1}}\langle\tau, \xi\rangle^{+} d \mu(\xi) \text { for all } \tau \in \mathbb{S}^{n-1} .
$$

If $h=h(\cdot, E(q))$ is the support function for $E(q)$ and $r \tau \in E(q)$ for some $r>0$ and $\tau \in \mathbb{S}^{n-1}$ then it follows from (11.7) and the definition of $h$ that

$$
0<\phi r \leq \int_{\mathbb{S}^{n-1}}\langle r \tau, \xi\rangle^{+} d \mu(\xi) \leq \int_{\mathbb{S}^{n-1}} h(\xi) d \mu(\xi) \leq \theta(q) .
$$

From (11.8) we first see that $E(q) \subset\{x:|x| \leq \theta(q) / \phi\}$. We then conclude the existence of $q^{l}=\left(q_{1}^{l}, \ldots, q_{m}^{l}\right), q_{i}^{l} \geq 0$ for $1 \leq i \leq m$ such that $E_{l}=E\left(q^{l}\right), l=3,4, \ldots$, is a sequence of uniformly bounded compact convex sets in the class $\Phi$ with

$$
\hat{q}=\lim _{l \rightarrow \infty} q^{l} \quad \text { and } \quad \lim _{l \rightarrow \infty} \theta\left(q^{l}\right)=\lambda=\theta(\hat{q}) .
$$

From finiteness of $\lambda$ we also may assume that $E_{l} \rightarrow E(\hat{q})=E_{1}$, a compact convex set containing 0, where convergence is uniform in the Hausdorff distance sense. From (11.1) we see that

$$
\lim _{l \rightarrow \infty} \mathcal{C}_{\mathcal{A}}\left(E_{l}\right)=\mathcal{C}_{\mathcal{A}}\left(E_{1}\right) .
$$

Thus $\mathcal{C}_{\mathcal{A}}\left(E_{1}\right) \geq 1$ and in fact $\mathcal{C}_{\mathcal{A}}\left(E_{1}\right)=1$. Otherwise, we would have $\theta(\tilde{q})<\theta(\hat{q})$ for $\tilde{E}=\tilde{E}(\tilde{q}) \in \Phi$ where for $j \in\{1,2, \ldots, m\}$,

$$
\tilde{q}_{j}= \begin{cases}\frac{\hat{q}_{j}}{\mathcal{C}_{\mathcal{A}}\left(E_{1}\right)} & \text { when } p=n, \\ \frac{\hat{q}_{j}}{\mathcal{C}_{\mathcal{A}}\left(E_{1}\right)^{1 /(p-n)}} & \text { when } p>n .\end{cases}
$$

If $E_{1}$ has nonempty interior, say $z$ is an interior point of $E_{1}$, then $\breve{E}=E_{1}-z \in \Phi$ and $\mathcal{C}_{\mathcal{A}}(\breve{E})=1$ as we deduce from translation invariance of $\mathcal{C}_{\mathcal{A}}(\cdot)$. Moreover, if $\breve{E}=E(\breve{q})$ then from (11.3) and $\theta(\hat{q})=\lambda$ we see that $\theta(\breve{q})=\lambda$. Thus, (11.6) is valid if $E_{1}$ has nonempty interior. 
If $E_{1}$ has empty interior, then from convexity of $E_{1}$ and (11.4) we find that $E_{1}$ is contained in an $l$-dimensional plane with $l<n-1$ and $0<\mathcal{H}^{l}\left(E_{1}\right)<\infty$. Also $E_{1}$ must contain at least two points since $\mathcal{C}_{\mathcal{A}}\left(E_{1}\right)=1$ so $l \geq 1$. We assume, as we may, that 0 is an interior point of $E_{1}$ relative to the $l$-dimensional plane containing $E_{1}$ since otherwise we consider $E_{1}-z$ for some $z$ having this property and argue as above. From the definition of $\Phi$ we see that there exists a subset, say $\Lambda$ of $\{1, \ldots, m\}$, with $\hat{q}_{i}=0$ when $i \in \Lambda$. Since $\hat{q}$ gives a minimum for $\theta$ we observe that if $s \notin \Lambda$, then $\hat{q}_{s} \neq 0$ and

$$
\left\{x:\left\langle x, \xi_{s}\right\rangle=\hat{q}_{s}\right\} \cap E_{1} \neq \emptyset .
$$

Let $a=\frac{1}{4} \min \left\{\hat{q}_{i}: i \notin \Lambda\right\}$ and for small $t>0$ let

$$
\tilde{E}(t)=\bigcap_{i=1}^{m}\left\{x:\left\langle x, \xi_{i}\right\rangle \leq \hat{q}_{i}+a t\right\} \quad \text { and } \quad E_{2}=\bigcap_{i=1}^{m}\left\{x:\left\langle x, \xi_{i}\right\rangle \leq a\right\} .
$$

Put

$$
E_{t}=\frac{\tilde{E}(t)}{\mathcal{C}_{\mathcal{A}}(\tilde{E}(t))} \text { when } p=n \text { and } \quad E_{t}=\frac{\tilde{E}(t)}{\mathcal{C}_{\mathcal{A}}(\tilde{E}(t))^{1 /(p-n)}} \text { when } p>n \text {. }
$$

We note that, in view of (11.11), $E_{t}=E(q(t))$ where $q(t)=\left(q_{1}(t), \ldots, q_{m}(t)\right)$ and for $j=1,2, \ldots, m$

$$
q_{j}(t)=\frac{\hat{q}_{j}+a t}{\mathcal{C}_{\mathcal{A}}(\tilde{E}(t))} \text { when } p=n \quad \text { and } \quad q_{j}(t)=\frac{\hat{q}_{j}+a t}{\mathcal{C}_{\mathcal{A}}(\tilde{E}(t))^{1 /(p-n)}} \text { when } p>n .
$$

From (5.23) we have $\mathcal{C}_{\mathcal{A}}\left(E_{t}\right)=1$ so $E_{t} \in \Phi$. To get a contradiction to our assumption that $E_{1}$ has empty interior we show that

$$
\theta(q(t))<\lambda \text { for some small } t>0 .
$$

To prove (11.13), we first note that $E_{1}+t E_{2} \subset \tilde{E}(t)$ for $t \in(0,1)$ so

$$
\mathcal{C}_{\mathcal{A}}\left(E_{1}+t E_{2}\right) \leq \mathcal{C}_{\mathcal{A}}(\tilde{E}(t))
$$

From this inequality and (11.11) and (11.12), we conclude that to prove (11.13) it suffices to show if

$$
\chi(t)= \begin{cases}{\left[\mathcal{C}_{\mathcal{A}}\left(E_{1}+t E_{2}\right)\right]^{-1} \sum_{i=1}^{m} c_{i}\left(\hat{q}_{i}+a t\right)} & \text { when } p=n, \\ {\left[\mathcal{C}_{\mathcal{A}}\left(E_{1}+t E_{2}\right)\right]^{-1 /(p-n)} \sum_{i=1}^{m} c_{i}\left(\hat{q}_{i}+a t\right)} & \text { when } p>n\end{cases}
$$

then

$$
\chi(t)<\lambda \text { for } t>0 \text { near } 0 \text {. }
$$

To prove (11.15), we let, as in section $10, u(\cdot, t)$ be the $\mathcal{A}=\nabla f$-harmonic Green's function for $\mathbb{R}^{n} \backslash\left(E_{1}+t E_{2}\right)$ with pole at infinity. We also let $\mathbf{g}\left(\cdot, E_{1}+t E_{2}\right)$ be the 
Gauss map for $\partial\left(E_{1}+t E_{2}\right)$ while $h_{1}$ and $h_{2}$ are the support functions for $E_{1}$ and $E_{2}$, respectively. Then from Remark 10.2 and Proposition 10.1 we have for $t \in(0,1)$,

$$
\begin{aligned}
& \frac{d}{d t} \mathcal{C}_{\mathcal{A}}\left(E_{1}+t E_{2}\right) \\
& = \begin{cases}n \gamma^{-1} \mathcal{C}_{\mathcal{A}}\left(E_{1}+t E_{2}\right) \int_{\partial\left(E_{1}+t E_{2}\right)} h_{2}\left(\mathbf{g}\left(x, E_{1}+t E_{2}\right)\right) f(\nabla u(x, t)) d \mathcal{H}^{n-1} & \text { when } p=n, \\
p(p-1) \mathcal{C}_{\mathcal{A}}\left(E_{1}+t E_{2}\right)^{\frac{p-2}{p-1}} \int_{\partial\left(E_{1}+t E_{2}\right)} h_{2}\left(\mathbf{g}\left(x, E_{1}+t E_{2}\right)\right) f(\nabla u(x, t)) d \mathcal{H}^{n-1} & \text { when } p>n .\end{cases}
\end{aligned}
$$

We shall prove

\section{Proposition 11.1.}

$$
\lim _{\tau \rightarrow 0} \int_{\partial\left(E_{1}+\tau E_{2}\right)} h_{2}\left(\mathbf{g}\left(x, E_{1}+\tau E_{2}\right)\right) f(\nabla u(x, \tau)) d \mathcal{H}^{n-1}=\infty .
$$

Assuming Proposition 11.1 we get (11.15) and so a contradiction to our assumption that $E_{1}$ has empty interior as follows. First observe from (11.16) and (11.14) that for $p=n$,

$$
\begin{aligned}
\left.\mathcal{C}_{\mathcal{A}}\left(E_{1}+t E_{2}\right) \frac{d}{d t} \chi(t)\right|_{t=\tau} \\
\quad=\sum_{i=1}^{m} c_{i} a-n \gamma^{-1}\left[\sum_{i=1}^{m} c_{i}\left(\hat{q}_{i}+a \tau\right)\right] \int_{\partial\left(E_{1}+\tau E_{2}\right)} h_{2}\left(\mathbf{g}\left(x, E_{1}+\tau E_{2}\right)\right) f(\nabla u(x, \tau)) d \mathcal{H}^{n-1}
\end{aligned}
$$

and if $p>n$,

$$
\begin{aligned}
& {\left.\left[\mathcal{C}_{\mathcal{A}}\left(E_{1}+t E_{2}\right)\right]^{\frac{2 p-(n+1)}{(p-n)(p-1)}} \frac{d}{d t} \chi(t)\right|_{t=\tau}=\mathcal{C}_{\mathcal{A}}\left(E_{1}+\tau E_{2}\right)^{1 /(p-1)} \sum_{i=1}^{m} c_{i} a} \\
& \quad-\frac{p(p-1)}{(p-n)}\left[\sum_{i=1}^{m} c_{i}\left(\hat{q}_{i}+a \tau\right)\right] \int_{\partial\left(E_{1}+\tau E_{2}\right)} h_{2}\left(\mathbf{g}\left(x, E_{1}+\tau E_{2}\right)\right) f(\nabla u(x, \tau)) d \mathcal{H}^{n-1} .
\end{aligned}
$$

Now $E_{1}+\tau E_{2} \rightarrow E_{1}$ as $\tau \rightarrow 0$ in the sense of Hausdorff distance so by (11.1), we have (for all $n \leq p<\infty$ )

$$
\lim _{\tau \rightarrow 0} \mathcal{C}_{\mathcal{A}}\left(E_{1}+\tau E_{2}\right)=\mathcal{C}_{\mathcal{A}}\left(E_{1}\right)=1 .
$$

Clearly, (11.17)-(11.20) imply for some $t_{0}>0$ small that

$$
\left.\frac{d}{d t} \chi(t)\right|_{t=\tau}<0 \quad \text { for } \tau \in\left(0, t_{0}\right] .
$$

On the other hand, from (11.14) and (11.20) we see that

$$
\lim _{\tau \rightarrow 0} \chi(\tau)=\lambda \text {. }
$$


From this observation, the mean value theorem from calculus, and (11.21) we conclude that (11.15) holds so $E_{1}$ has interior points. Now (11.6) follows from our earlier remarks.

Proof of Proposition 11.1. Recall that $E_{1}$ is contained in a $1 \leq l<n-1$ dimensional plane. We assume as we may that

$$
E_{1} \subset\left\{x=\left(x^{\prime}, x^{\prime \prime}\right): x^{\prime}=\left(x_{1}, \ldots, x_{l}\right) \text { and } x^{\prime \prime}=\left(x_{l+1}, \ldots x_{n}\right)=(0, \ldots, 0)\right\}=\mathbb{R}^{l} .
$$

Indeed, otherwise we can rotate our coordinate system to get (11.22) and corresponding $\hat{\mathcal{A}}$-harmonic Green's functions, say $\bar{u}(\cdot, t)$ for $\mathbb{R}^{n} \backslash E_{1}$ with a pole at infinity. Proving Proposition 11.1 for $\bar{u}(\cdot, t)$ and transferring back we obtain Proposition 11.1.

We also note that

$$
\bar{B}(0,4 a) \cap \mathbb{R}^{l} \subset E_{1} \subset E_{1}+E_{2} \subset \bar{B}(0, \rho)
$$

which follows from our choice of $a$ and for some $\rho>0$ depending only on the data, since $\mathcal{C}_{\mathcal{A}}\left(E_{1}\right)=1$. Next we observe from Lemma 5.3 in [LN4] that for fixed $p>n-l$, there exists an $\mathcal{A}$-harmonic function $\hat{V}$ on $\mathbb{R}^{n} \backslash \mathbb{R}^{l}$ with continuous boundary value 0 on $\mathbb{R}^{l}$ satisfying

$$
\hat{V}(x) \approx\left|x^{\prime \prime}\right|^{\psi} \quad \text { whenever } x=\left(x^{\prime}, x^{\prime \prime}\right) \in \mathbb{R}^{l} \times \mathbb{R}^{n-l} \text { where } \psi=\frac{p-n+l}{p-1} .
$$

Ratio constants depend only on $p, n, l$ and the structure constants for $\mathcal{A}$. Using (11.24) we prove

Lemma 11.2. Fix $p \geq n$. Then there exists $C_{1} \geq 10^{10}$, depending on $p, n, l$, and $\rho$ but independent of $t \in(0,1]$, such that if $\psi=(p-n+l) /(p-1)$ and $x=\left(x^{\prime}, x^{\prime \prime}\right) \in B(0, \rho)$ then

$$
\left|x^{\prime \prime}\right|^{\psi} \leq C_{1} u(x, t) \quad \text { whenever } \quad C_{1} t \leq\left|x^{\prime \prime}\right|
$$

Proof of Lemma 11.2. For fixed $t \in(0,1)$, let $v=\max \left(\hat{V}-C_{2} t, 0\right)$ for $C_{2}>0$ which will be fixed soon. Then $v$ is $\mathcal{A}$-harmonic in $\mathbb{R}^{n} \backslash W$ and continuous on $\mathbb{R}^{n}$ with $v \equiv 0$ on $W=\left\{x: \hat{V}(x) \leq C_{2} t\right\}$. From the definition of $E_{1}+t E_{2}$ and $v$ we see for $C_{2}$ large enough, depending on $p, n, l$, the structure constants for $\mathcal{A}$, and $\rho$, that $v=0$ on $E_{1}+t E_{2}$. Also $C_{3} u(\cdot, t) \geq v$ on $\partial B(0,2 \rho)$ for $t \in[0,1]$ as we deduce from Harnack's inequality and $E_{1}+E_{2} \subset B(0, \rho)$. Here $C_{3}$ has the same dependence as $C_{2}$. Using the maximum principle for $\mathcal{A}$-harmonic functions it now follows that $\left.v \leq C_{4} u(\cdot, t)\right)$ in $B(0,2 \rho)$. From this fact and our knowledge of $\hat{V}$ we get Lemma 11.2.

To begin the proof of Proposition 11.1 we assume $0<t \leq \tilde{t}_{0}$, where $\tilde{t}_{0}<<a$. We also observe that $E_{1}+t E_{2}$ is a compact convex set with nonempty interior. From (8.6) (b) we find that for $\mathcal{H}^{n-1}$-almost every $\hat{x} \in \partial\left(E_{1}+t E_{2}\right)$

$$
\nabla u(y, t) \rightarrow \nabla u(\hat{x}, t) \quad \text { as } \quad y \rightarrow \hat{x}
$$


non-tangentially in $\mathbb{R}^{n} \backslash\left(E_{1}+t E_{2}\right)$. Moreover, there exists $\tilde{c}$ such that $B(\hat{x}, 4 t / \tilde{c}) \cap$ $\partial\left(E_{1}+t E_{2}\right)$ is the graph of a Lipschitz function whenever

$$
\hat{x} \in B(0,2 a) \cap \partial\left(E_{1}+t E_{2}\right) \quad \text { and } \quad 0<t \leq \tilde{t}_{0}
$$

with Lipschitz constant independent of $\hat{x}, t$. It then follows from (8.5), (8.3), (8.8), and (8.9) (a) with $q=p$ that

$$
\begin{aligned}
c \int_{B(\hat{x}, t / \tilde{c}) \cap \partial\left(E_{1}+t E_{2}\right)} f(\nabla u(\cdot, t)) d \mathcal{H}^{n-1} & \geq(u(w, t))^{p} t^{n-1-p} \\
& \geq c^{-1} \int_{B(\hat{x}, t / \tilde{c}) \cap \partial\left(E_{1}+t E_{2}\right)} f(\nabla u(\cdot, t)) d \mathcal{H}^{n-1}
\end{aligned}
$$

where $c$ has the same dependence as in Lemma 8.2. Also $w=w(\hat{x}, t)$ denotes a point in $B(\hat{x}, t / \tilde{c}) \cap\left(\mathbb{R}^{n} \backslash\left(E_{1}+t E_{2}\right)\right)$ whose distance from $\partial\left(E_{1}+t E_{2}\right)$ is $\geq t / c^{2}$.

Using Harnack's inequality in a chain of balls of radius $\approx t$ connecting $w$ to a point $x \in B(0, a)$ with $2 C_{1} t=\left|x^{\prime \prime}\right|$ we deduce from (11.25) of Lemma 11.2 that

$$
u(w, t) \geq C^{-1} t^{\psi}
$$

where $C$ is independent of $t \in(0,1)$. Using (11.27) in (11.26) we obtain for some $C^{\prime} \geq 1$, independent of $t, 0<t \leq \tilde{t}_{0}$, that

$$
C^{\prime} \int_{B(\hat{x}, t / \tilde{c}) \cap \partial\left(E_{1}+t E_{2}\right)} f(\nabla u(\cdot, t)) d \mathcal{H}^{n-1} \geq t^{p(\psi-1)+n-1} .
$$

Now since $\partial\left(E_{1}+t E_{2}\right) \cap B(0,2 a)$ projects onto a set containing $B(0,2 a) \cap \mathbb{R}^{l}$ for $0<t \leq \tilde{t}_{0}$, we see there is a disjoint collection of balls $B(\hat{x}, t / \tilde{c})$ for $\hat{x} \in \partial\left(E_{1}+t E_{2}\right)$ of cardinality approximately $t^{-l}$ for which (11.28) holds. Since

$$
p(\psi-1)+(n-1)-l=(l+1-n) /(p-1)<0
$$

we conclude from (11.28) that for some $C^{*}$ independent of small positive $t$

$$
C^{*} \int_{\partial\left(E_{1}+t E_{2}\right) \cap B(0,2 a)} f(\nabla u(\cdot, t)) d \mathcal{H}^{n-1} \geq t^{(l+1-n) /(p-1)} \rightarrow \infty \quad \text { as } \quad t \rightarrow 0 .
$$

Finally, note that for $0<t \leq \tilde{t}_{0}$,

$$
\mathbf{g}\left(x, E_{1}+t E_{2}\right) \in\left\{\xi_{i}: i \in \Lambda\right\}
$$

for $\mathcal{H}^{n-1}$-almost every $x \in \partial\left(E_{1}+t E_{2}\right) \cap B(0,2 a)$ and $h_{2}\left(\xi_{i}\right) \equiv a$ whenever $\xi_{i} \in \Lambda$. From this note and (11.29), we obtain first the validity of (11.15) in Proposition 11.1 and thereupon that (11.6) is true.

Armed with (11.6), we can use the well-known variational argument mentioned after this display to complete the proof of existence in Theorem B in the discrete case. To do so we first observe that if $E_{1}$ and $E_{2}$ are compact convex sets with 
nonempty interiors and if $h_{1}$ and $h_{2}$ are the support functions for these sets with the corresponding $\mathcal{A}$-harmonic Green's functions $u_{1}$ and $u_{2}$ then

$$
\begin{aligned}
& \left.\frac{d}{d s} \mathcal{C}_{\mathcal{A}}\left((1-s) E_{1}+s E_{2}\right)\right|_{s=0} \\
& \quad= \begin{cases}n \gamma^{-1} \mathcal{C}_{\mathcal{A}}\left(E_{1}\right) \int_{\partial E_{1}}\left(h_{2}-h_{1}\right)\left(\mathbf{g}\left(x, E_{1}\right)\right) f\left(\nabla u_{1}(x)\right) d \mathcal{H}^{n-1} & \text { when } p=n \\
p(p-1) \mathcal{C}_{\mathcal{A}}\left(E_{1}\right)^{\frac{p-2}{p-1}} \int_{\partial E_{1}}\left(h_{2}-h_{1}\right)\left(\mathbf{g}\left(x, E_{1}\right)\right) f\left(\nabla u_{1}(x)\right) d \mathcal{H}^{n-1} & \text { when } p>n\end{cases}
\end{aligned}
$$

Here (11.30) follows from Proposition 10.1, (10.36), (10.37), and the chain rule using $t=s /(1-s)$ and

$$
\mathcal{C}_{\mathcal{A}}\left((1-s) E_{1}+s E_{2}\right)= \begin{cases}(1-s) \mathcal{C}_{\mathcal{A}}\left(E_{1}+t E_{2}\right) & \text { when } p=n \\ (1-s)^{p-n} \mathcal{C}_{\mathcal{A}}\left(E_{1}+t E_{2}\right) & \text { when } p>n\end{cases}
$$

Let $q^{*}=\left(q_{1}^{*}, \ldots, q_{m}^{*}\right) \in \mathbb{R}^{m}$ with $q_{i}^{*}>0$ for $1 \leq i \leq m$ and $\breve{q}$ as in (11.6). From the note after (11.13) and (11.6) we deduce for $\bar{t}_{0}>0$ sufficiently small, that $E\left(q^{*}(t)\right) \in \Phi$ when $0<t \leq \bar{t}_{0}$, where

$$
q^{*}(t)= \begin{cases}\frac{(1-t) \breve{q}+t q^{*}}{\mathrm{C}_{\mathcal{A}}\left((1-t) E(\breve{q})+t E\left(q^{*}\right)\right)} & \text { when } p=n \\ \frac{(1-t) \breve{q}+t q^{*}}{\mathrm{C}_{\mathcal{A}}\left((1-t) E(\breve{q})+t E\left(q^{*}\right)\right)^{1 /(p-n)}} & \text { when } p>n\end{cases}
$$

Also, $\theta\left(q^{*}(t)\right) \geq \lambda$ for $0 \leq t \leq \bar{t}_{0}$ thanks to (11.6). Differentiating $\theta\left(q^{*}(t)\right)$ with respect to $t$ and evaluating at 0 we obtain from (11.30) with $E_{1}=E(\breve{q}), E_{2}=E\left(q^{*}\right)$, and $u_{1}$ the $\mathcal{A}$-harmonic Green's function for $\mathbb{R}^{n} \backslash E(\breve{q})$, as well as (11.6) and the fact that $\mathcal{C}_{\mathcal{A}}\left(E_{1}\right)=1$ by $(11.20)$ that when $p=n$,

$$
\begin{aligned}
0 & \leq\left.\frac{d \theta\left(q^{*}(\tau)\right)}{d \tau}\right|_{\tau=0} \\
& =\sum_{i=1}^{m} c_{i}\left(q_{i}^{*}-\breve{q}_{i}\right)-n \lambda \gamma^{-1} \int_{\partial E(\breve{q})}\left(h_{2}-h_{1}\right)(\mathbf{g}(x, E(\breve{q}))) f\left(\nabla u_{1}(x)\right) d \mathcal{H}^{n-1} \\
& =\sum_{i=1}^{m} c_{i}\left(q_{i}^{*}-\breve{q}_{i}\right)-n \lambda \gamma^{-1} \sum_{i=1}^{m}\left(q_{i}^{*}-\breve{q}_{i}\right) \int_{\mathbf{g}^{-1}\left(\xi_{i}, E(\breve{q})\right)} f\left(\nabla u_{1}(x)\right) d \mathcal{H}^{n-1}
\end{aligned}
$$


provided $q^{*}$ is near enough $\hat{q}$. Similarly, if $p>n$,

$$
\begin{aligned}
0 & \leq\left.\frac{d \theta\left(q^{*}(\tau)\right)}{d \tau}\right|_{\tau=0} \\
& =\sum_{i=1}^{m} c_{i}\left(q_{i}^{*}-\breve{q}_{i}\right)-p \lambda \frac{p-1}{p-n} \int_{\partial E(\breve{q})}\left(h_{2}-h_{1}\right)(\mathbf{g}(x, E(\breve{q}))) f\left(\nabla u_{1}(x)\right) d \mathcal{H}^{n-1} \\
& =\sum_{i=1}^{m} c_{i}\left(q_{i}^{*}-\breve{q}_{i}\right)-p \lambda \frac{p-1}{p-n} \sum_{i=1}^{m}\left(q_{i}^{*}-\breve{q}_{i}\right) \int_{\mathbf{g}^{-1}\left(\xi_{i}, E(\breve{q})\right)} f\left(\nabla u_{1}(x)\right) d \mathcal{H}^{n-1}
\end{aligned}
$$

From arbitrariness of $\breve{q}_{i}-q_{i}^{*}$ we conclude that

$$
c_{i}=b \int_{\mathbf{g}^{-1}\left(\xi_{i}, E(\breve{q})\right)} f\left(\nabla u_{1}(x)\right) d \mathcal{H}^{n-1} \quad \text { for } 1 \leq i \leq m .
$$

where

$$
b= \begin{cases}n \lambda / \gamma & \text { when } p=n \\ p \lambda \frac{p-1}{p-n} & \text { when } p>n .\end{cases}
$$

Observe that $\lambda>0$ since otherwise $E(\breve{q})=\{0\}$ a contradiction to $\mathcal{C}_{\mathcal{A}}(E(\breve{q}))=1$. From (11.33) and $p$-homogeneity of $f$ we find that if $p \geq n$ and $E=\delta E(\breve{q})$ where $\delta^{-1}=n \lambda / \gamma$ when $p=n$ while $\delta^{n-p-1}=p\left(\frac{p-1}{p-n}\right) \lambda$ for $p>n$, then (11.5) holds. This completes the proof of existence in the discrete case when (11.2)-(11.4) are valid.

Remark 11.3. For later use we note from (10.36) and (10.37) with $E_{0}=E$, that if $h$ is the support function for $E$ as in (7.2) when $p \geq n$, then

$$
p \int_{\mathbb{S}^{n-1}} h(\xi) d \mu(\xi)= \begin{cases}\gamma & \text { when } p=n \\ \frac{p-n}{p-1} \mathcal{C}_{\mathcal{A}}(E)^{1 /(p-1)} \leq c(\operatorname{diam}(E))^{\frac{p-n}{p-1}} & \text { when } p>n\end{cases}
$$

11.2. Existence in Theorem B in the continuous case. Armed with existence in Theorem $\mathrm{B}$ in the discrete case when $p \geq n$, we now consider existence when $\mu$ is a finite positive Borel measure on $\mathbb{S}^{n-1}$ satisfying (7.1). We choose a sequence of discrete measures $\left\{\mu_{j}\right\}_{j \geq 1}$ satisfying (11.2)-(11.4) when $p \geq n$ is fixed with

$$
\mu_{j} \rightarrow \mu \text { weakly as } j \rightarrow \infty \text {. }
$$

Let $E_{j}, j=1,2, \ldots$, be a corresponding sequence of compact convex sets with 0 in the interior of $E_{j}$ and corresponding $\mathcal{A}$-harmonic Green's functions $U_{j}$ for $\mathbb{R}^{n} \backslash E_{j}$ with pole at infinity for which (11.5) holds at support points of $\mu_{j}$. From the definition of weak convergence we may assume for some $C \geq 1$ that

$$
C^{-1} \leq \mu_{j}\left(\mathbb{S}^{n-1}\right) \leq C \text { for } j=1,2, \ldots
$$

We claim that we may also assume

$$
E_{j} \subset \bar{B}(0, \rho) \text { for } j=1,2, \ldots \text {, and some } \rho<\infty \text {. }
$$


To prove (11.36) we observe from weak convergence of $\left(\mu_{j}\right)$ to $\mu$ and (11.2), (11.3), that for some $\hat{C} \geq 1$, independent of $j=1,2, \ldots$,

$$
\hat{C}^{-1} \leq \int_{\mathbb{S}^{n-1}}\langle\tau, \xi\rangle^{+} d \mu_{j}(\xi) \text { for all } \tau \in \mathbb{S}^{n-1}
$$

Using (11.37), (11.34), and arguing as in (11.8) we deduce that if $\hat{\tau}_{j} \in \mathbb{S}^{n-1}$ so that $\hat{s}_{j} \hat{\tau}_{j} \in E_{j}$ with $\hat{s}_{j} \geq \frac{1}{10} \operatorname{diam}\left(E_{j}\right)$ and $h_{j}$ is the support function for $E_{j}$ then

$$
\hat{C}^{-1} \hat{s}_{j} \leq \int_{\mathbb{S}^{n-1}}\left\langle\hat{s}_{j} \hat{\tau}_{j}, \xi\right\rangle^{+} d \mu_{j}(\xi) \leq \int_{\mathbb{S}^{n-1}} h_{j}(\xi) d \mu_{j}(\xi) \leq \tilde{C} s_{j}^{\frac{p-n}{p-1}}
$$

where all constants are positive and independent of $j$. Thus, claim (11.36) is true.

From (11.36) we see that a subsequence of $\left\{E_{j}\right\}_{j \geq 1}$ (also denoted $\left\{E_{j}\right\}$ ) converges to a compact convex set $E \subset \bar{B}(0, \rho)$ in the sense of Hausdorff distance. Choosing another subsequence if necessary we may assume from the same argument used in proving (11.1) that either $E=\{0\}$ or $U_{j} \rightarrow U$ uniformly in $\mathbb{R}^{n}$ where $U$ is the $\mathcal{A}$ harmonic Green's function for $\mathbb{R}^{n} \backslash E$ with pole at infinity. If $E$ has nonempty interior then from Proposition 9.1 we conclude that (7.2) (b) and $(c)$ hold for $U, E$, and $\mu$.

If $E$ has empty interior we consider the following three cases :

Case A: $E$ has dimension $l, 1 \leq l<n-1$. In this case we once again use the argument in [AGHLV, section 13.2, Case B1] to get a contradiction. Translating $E$ if necessary we may assume that 0 is an interior point of the $l$ dimensional plane $P$ containing $E$. So as in the discrete case we assume that

$$
B(0,4 a) \cap P \subset E \subset B(0, \rho) \cap E
$$

and

$$
t_{j}=d_{\mathcal{H}}\left(E_{j}, E\right) \quad \text { for } j=1,2, \ldots
$$

Then for $j$ large enough we can argue as in Lemma 11.2 with $t$ replaced by $t_{j}$, and $u(\cdot, t), E_{1}+t E_{2}$ by $U_{j}, E_{j}$. We obtain from the analogue of (11.25) for $j \geq j_{0}$, that

$$
U_{j}(x) \geq C_{1}^{-1}\left|x^{\prime \prime}\right|^{\psi} \quad \text { for } x=\left(x^{\prime}, x^{\prime \prime}\right) \in B(0, \rho) \text { and } C_{1} t_{j} \leq\left|x^{\prime \prime}\right|
$$

where $x^{\prime} \in \mathbb{R}^{l}$ and $\psi=(p-n+l) /(p-1)$. Fix $j \geq j_{0}$, and given $y \in \partial E_{j} \cap B(0, a)$, let $T_{j}(y)$, be a supporting hyperplane to $\partial E_{j}$ at $y$. Let $\hat{H}_{j}$ be the open half space with $\hat{H}_{j} \cap E_{j}=\emptyset$ and $\partial \hat{H}_{j}=T_{j}(y)$. Let $y^{*}$ denote the point in $\hat{H}_{j}$ which lies on the normal line through $y$ with $\left|y-y^{*}\right|=2 C_{1} t_{j}$ where $C_{1}$ is as in (11.40). Note that for $j_{0}$ sufficiently large and $j \geq j_{0}$ that $d\left(y^{*}, P\right)>C_{1} t_{j}$, since otherwise it would follow from the triangle inequality that there exists $z \in B(0,2 a) \cap E$ with $d\left(z, E_{j}\right)>t_{j}$. Thus (11.40) holds with $x=y^{*}$. Let $\phi$ be the $\mathcal{A}$-harmonic function in $\hat{H}_{j} \cap B\left(y, 8 C_{1} t_{j}\right) \backslash \bar{B}\left(y^{*}, C_{1} t_{j}\right)$ with continuous boundary values

$$
\phi \equiv \begin{cases}U_{j} & \text { on } \partial B\left(y^{*}, C_{1} t_{j}\right) \\ 0 & \text { on } \partial\left(\hat{H}_{j} \cap B\left(y, 8 C_{1} t_{j}\right)\right) .\end{cases}
$$

Then from the maximum principle for $\mathcal{A}$-harmonic functions we have $\phi \leq U_{j}$ on $\hat{H}_{j} \cap B\left(y, 8 C_{1} t_{j}\right) \backslash B\left(y^{*}, C_{1} t_{j}\right)$. Comparing $\phi$ to a linear function and using a boundary 
Harnack inequality from [LLN] in $\hat{H}_{j} \cap B\left(y, 8 C_{1} t_{j}\right)$ we deduce for some $c^{*}$ depending only on the data and $\rho$ that

$$
\left.\left.U_{j}\left(y^{*}\right)\right) / t_{j} \leq c^{*} U_{j}(\hat{z})\right) / d\left(\hat{z}, T_{j}(y)\right)
$$

when $j \geq j_{0}$ and $\hat{z} \in \hat{H}_{j} \cap B\left(y, C_{1} t_{j}\right)$. Letting $\hat{z} \rightarrow y$ non-tangentially, we conclude from this inequality and (11.40) with $x=y^{*}$ that

$$
t_{j}^{\psi-1} \leq C^{* *}\left|\nabla U_{j}(y)\right| \text { for } \mathcal{H}^{n-1} \text {-almost every } y \in \partial E_{j} \cap B(0, a)
$$

and $j \geq j_{0}$. Here $C^{* *}$ has the same dependence as $C_{1}$.

Let $\nu_{j}$ be the positive Borel measure corresponding to $U_{j}$ with support contained in $E_{j}$ as in Lemmas 5.2. 5.3. Then from (8.8) we deduce for $j=1,2, \ldots$ that

$$
\frac{d \nu_{j}}{d \mathcal{H}^{n-1}}(y)=p \frac{f\left(\nabla U_{j}(y)\right)}{\left|\nabla U_{j}(y)\right|} \text { for } \quad \mathcal{H}^{n-1} \text {-almost every } y \in \partial E_{j} \text {. }
$$

From (11.42), the definition of $\mu_{j}$, the structure assumptions on $f$, and (11.41) we conclude for some $\breve{C} \geq 1$, independent of $j \geq j_{0}$, that for fixed $p \geq n$

$$
t_{j}^{\psi-1} \nu_{j}\left(\partial E_{j} \cap B(0, a)\right) \leq C^{\prime} \mu_{j}\left(\mathbf{g}\left(\partial E_{j} \cap B(0, a), E_{j}\right)\right) .
$$

Here $\mathbf{g}\left(\cdot, E_{j}\right)$ is the Gauss map for $\partial E_{j}$. Now $\psi-1=(1-n+k) /(p-1)<0$ and from weak convergence of $\left(\nu_{j}\right)$ to $\nu$ we have

$$
\liminf _{j \rightarrow \infty} \nu_{j}\left(\partial E_{j} \cap B(0, a)\right) \geq \nu(\partial E \cap B(0, a / 2))>0
$$

where the right-hand inequality follows from the fact that $E \cap B(0, a / 2)$ is uniformly $(a / 2, p)$-fat. Putting this inequality in $(11.43)$ we see that $\mu_{j}\left(\mathbb{S}^{n-1}\right) \rightarrow \infty$ in contradiction to (11.35). Thus $E$ does not have dimension $l, 1 \leq l<n-1$.

Case B: $E=\{0\}$. In this case we put $t_{j}=\operatorname{diam}\left(E_{j}\right)$ and let $\nu_{j}$ be the positive Borel measure, as above, relative to $U_{j}$ with support contained in $E_{j}$ as in Lemma 3.7. Then from $(d)$ of Lemmas 5.2 and 5.3 we have $\nu_{j}\left(E_{j}\right)=1$ for $j=1,2, \ldots$ From this fact and (3.5) $(i i)$ we see that

$$
U_{j} \approx t_{j}^{\frac{p-n}{p-1}} \text { on } \partial B\left(0,2 t_{j}\right) \text { for } j=1,2, \ldots
$$

where ratio constants depend only on the data. Using (11.44) and the same argument as in the derivation of (11.41) it follows that for some $c \geq 1$ depending only on the data,

$$
\left|\nabla U_{j}(y)\right| \geq c^{-1} t_{j}^{\frac{1-n}{p-1}} \quad \text { for } \mathcal{H}^{n-1} \text {-almost every } y \in \partial E_{j} .
$$

Since $\nu_{j}\left(E_{j}\right)=1$ for $j=1,2, \ldots$, we again obtain as in Case $\mathbf{A}$ that

$$
c \mu_{j}\left(\partial E_{j}\right) \geq t_{j}^{\frac{1-n}{p-1}} \rightarrow \infty \quad \text { as } j \rightarrow \infty
$$

in contradiction to (11.35). So $E \neq\{0\}$.

Case C: $E$ has dimension $n-1$. In this case, we essentially copy the proof from [AGHLV] through the statement of Proposition 11.5. However to prove Proposition 
11.5 we state and prove Lemma 11.6 which in [AGHLV] was only available when $f(\eta)=p^{-1}|\eta|^{p}$, i.e, for the $p$-Laplace equation. Using Lemma 11.6 we can then copy the so called simple proof given in [AGHLV] of (11.52) for the $p$-Laplace equation when $2<p<n$.

To begin the proof we assume, as we may, that $P=\left\{x: x_{n}=0\right\}$ and

$$
B(0,4 a) \cap P \subset E \subset B(0, \rho) \cap P .
$$

Also translating $E_{j}$ slightly upward if necessary we may assume that

$$
\lim _{j \rightarrow \infty} d_{\mathcal{H}}\left(E_{j}, E\right)=0 \text { and } E_{j} \subset\left\{x: x_{n}>0\right\} .
$$

Let $\nabla U_{+}(x)$ denote the limit (whenever it exists) as $y \rightarrow x \in E$ non-tangentially through values with $y_{n}>0$. We prove

Proposition 11.4. There exists $C \geq 1$ such that

$$
C \liminf _{j \rightarrow \infty} \int_{\partial E_{j}} f\left(\nabla U_{j}\right) d \mathcal{H}^{n-1} \geq \int_{E} f\left(\nabla U_{+}\right) d \mathcal{H}^{n-1}-C^{2} \mathcal{H}^{n-1}(E) .
$$

Proof. Given $\epsilon>0$ choose $j_{1}$ so large that $d_{\mathcal{H}}\left(E_{j}, E\right) \leq \epsilon$ for $j \geq j_{1}$. Using uniform convergence of $\left(U_{j}\right)$ to $U$ on $\mathbb{R}^{n}$ and comparing boundary values of $U$ and $U_{j}$ in $B(0,2 \rho) \backslash E_{j}$, we deduce from Lemma 3.5 that there exist $0<\tilde{\sigma} \leq 1 / 2$ and $\hat{C} \geq 1$ such that

$$
U \leq \hat{C}\left(U_{j}+\epsilon^{\tilde{\sigma}}\right)
$$

for $j \geq j_{1}$. Next we divide the interior of $E$ into $(n-1)$-dimensional closed Whitney cubes $\left\{Q_{k}\right\}$. Let $\partial^{\prime} E$ denote the boundary of $E$ considered as a set in $P$ and let $d^{\prime}(\cdot, \cdot)$ denote the distance between sets considered in $P$. Then the cubes in $\left\{Q_{k}\right\}$ have disjoint interiors with side length $s\left(Q_{k}\right)$ and the property that considered as sets in $P$, the distance say $d^{\prime}\left(Q_{k}, \partial^{\prime} E\right)$ from $Q_{k}$ to the boundary of $E$ satisfies

$$
10^{-n} s\left(Q_{k}\right) \leq d^{\prime}\left(Q_{k}, \partial^{\prime} E\right) \leq 10^{n} s\left(Q_{k}\right) .
$$

Let $Q \in\left\{Q_{k}\right\}$ with $s(Q) \geq \epsilon^{\tilde{\sigma}}$ and put $Q_{+}=Q \times(0, s(Q))$. Suppose $y_{Q}=\left(y_{1}, \ldots, y_{n}\right)$ is a point in $Q_{+} \backslash E_{j}$ with $d\left(y_{Q}, \partial^{\prime} E\right) \geq y_{n} / 2 \geq s(Q) / 4$.

We consider two possibilities. If $U\left(y_{Q}\right) \geq 2 \hat{C} \epsilon^{\tilde{\sigma}}(\hat{C}$ as in (11.47)), then from (11.47) we have $U\left(y_{Q}\right) \leq 2 \hat{C} U_{j}\left(y_{Q}\right)$ and using (8.5) (b), (8.8), (8.9) (a), for $U_{j}, U$ we get

$$
\begin{aligned}
\bar{C}^{3} \int_{\partial E_{j} \cap Q_{+}} f\left(\nabla U_{j}\right) d \mathcal{H}^{n-1} & \geq \bar{C}^{2}\left(U_{j}\right)^{p}\left(y_{Q}\right) s(Q)^{n-1-p} \\
& \geq \bar{C}(U)^{p}\left(y_{Q}\right) s(Q)^{n-1-p} \\
& \geq \int_{Q} f\left(\nabla U_{+}\right) d \mathcal{H}^{n-1} .
\end{aligned}
$$

If $U\left(y_{Q}\right)<2 \hat{C} \epsilon^{\tilde{\sigma}}$, then since $s(Q) \geq \epsilon^{\tilde{\sigma}}$, an argument similar to the above gives

$$
\int_{Q} f\left(\nabla U_{+}\right) d \mathcal{H}^{n-1} \leq C_{+} s(Q)^{n-1}
$$


where $C_{+}$is independent of $j \geq j_{2} \geq j_{1}$ provided $j_{2}$ is large enough. Combining (11.49), (11.50), and using (11.48) we find after summing over $Q \in\left\{Q_{k}\right\}$ that for some $\breve{C} \geq 1$, independent of $j \geq j_{2}$,

$$
\breve{C} \int_{\partial E_{j}} f\left(\nabla U_{j}\right) d \mathcal{H}^{n-1} \geq \int_{\left\{x \in E: d^{\prime}\left(x, \partial^{\prime} E\right) \geq \breve{C} \epsilon^{\tilde{\sigma}}\right\}} f\left(\nabla U_{+}\right) d \mathcal{H}^{n-1}-\breve{C}^{2} \mathcal{H}^{n-1}(E) .
$$

Letting first $j \rightarrow \infty$ and after that $\epsilon \rightarrow 0$ we obtain from (11.51) and the monotone convergence theorem or Fatou's Lemma that (11.46) is true. This finishes the proof of Proposition 11.4.

Next we prove

\section{Proposition 11.5.}

$$
\int_{E} f\left(\nabla U_{+}\right) d \mathcal{H}^{n-1}=\infty
$$

We note that Propositions 11.4 and 11.5 give a contradiction to (11.35). From this contradiction we conclude first that $E$ does not have dimension $n-1$. From our previous work it now follows that $E$ is a compact convex set with nonempty interior and (7.2) $(a)-(c)$ are valid with $u=U$. This finishes the proof of existence in Theorem B up to proving Proposition 11.5. To prove Proposition 11.5 we shall need

Lemma 11.6. Given $\hat{\eta}=\left(\hat{\eta}_{1}, \hat{\eta}_{2}\right) \in \mathbb{R}^{2} \backslash\{0\}$, put $\tilde{f}(\hat{\eta})=f\left(\hat{\eta}_{1}, \hat{\eta}_{2}, 0, \ldots, 0\right)$. Then there exists a continuous function $v$ on $\mathbb{R}^{2}$ that is $\tilde{\mathcal{A}}:=\nabla \tilde{f}$-harmonic in $\mathbb{R}^{2} \backslash\{x=$ $\left.\left(x_{1}, 0\right) \in \mathbb{R}^{2}: x_{1} \leq 0\right\}$ with $v \equiv 0$ on $\left\{\left(x_{1}, 0\right) \in \mathbb{R}^{2}: x_{1} \leq 0\right\}$. Moreover, $v(1,0)=1$ and

$$
v(t x)=t^{1-1 / p} v(x) \quad \text { whenever } t>0 \text { and } x \in \mathbb{R}^{2} .
$$

Proof. We point out that Krol in $[\mathrm{Kr}]$ proved Lemma 11.6 when $\tilde{f}(\hat{\eta})=p^{-1}|\hat{\eta}|^{p}$, so that $v$ in this case is a solution to the $p$-Laplace equation. We introduce polar coordinates, $r=|x|, x_{1}=r \cos \theta, x_{2}=r \sin \theta$, and put

$$
D(\tau)=\{x: r>0,|\theta|<\tau\} \text { for } \pi / 2 \leq \tau \leq \pi .
$$

We claim for $\pi / 2 \leq \tau<\pi$ that there exists a unique positive $\tilde{\mathcal{A}}=\nabla \tilde{f}$-harmonic function $w=w(\cdot, \tau)$ in $D(\tau)$ which is continuous in $\mathbb{R}^{2}$ with $w \equiv 0$ on $\mathbb{R}^{2} \backslash D(\tau)$ and $w(1,0)=1$. Moreover,

$$
w(t x)=t^{\lambda} w(x) \quad \text { whenever } x \in \mathbb{R}^{2}
$$

for some $\lambda=\lambda(\tau)>0$. To construct $w$ for fixed $\tau$ with $\pi / 2 \leq \tau<\pi$, let $w_{l}$ be the continuous function in $\bar{B}(0,2 l)$ with $w_{l}$ an $\tilde{\mathcal{A}}=\nabla \tilde{f}$-harmonic function in $B(0,2 l) \backslash\left[\left(\mathbb{R}^{2} \backslash D(\tau)\right) \cap \bar{B}(0, l)\right]$ and $w_{l} \equiv 0$ on $\left(\mathbb{R}^{2} \backslash D(\tau)\right) \cap \bar{B}(0, l)$ while $w_{l}=M_{l}$ on $\partial B(0,2 l)$ where $M_{l}$ is chosen so that $w_{l}(1,0)=1$. Using Lemmas 3.3-3.5 and taking limits of a certain subsequence of $\left\{w_{l}\right\}_{l \geq 1}$, we see there exists $w \geq 0$, a Hölder continuous function on $\mathbb{R}^{2}$ which is $\tilde{\mathcal{A}}=\nabla \tilde{f}$-harmonic in $D(\tau)$ for fixed $p \geq 2$ with $w \equiv 0$ on $\mathbb{R}^{2} \backslash D(\tau)$ and $w(1,0)=1$. Uniqueness of $w$ follows from (8.10) applied in $D(\tau) \cap B\left(0,2 r^{+}\right)$to $w$ and $w^{\prime}$ where $w^{\prime}$ has the same properties as $w$ (and $r^{+}$ 
is as in Lemma 8.3). Letting $r^{+} \rightarrow \infty$, we conclude first that $w / w^{\prime}$ is bounded in $D(\tau)$ and after that from Hölder continuity of the ratio that $w^{\prime}=w$. To prove (11.54) observe that $w(t x)$ for $x \in \mathbb{R}^{2}$ is also $\tilde{\mathcal{A}}=\nabla \tilde{f}$-harmonic in $D(\tau)$ and $\equiv 0$ on $\mathbb{R}^{2} \backslash D(\tau)$ as follows from $p$-homogeneity of $\tilde{f}$. From uniqueness of $w$ we conclude that $w(t x)=w(t, 0) w(x)$. Differentiating this expression with respect to $t$ and evaluating at $t=1$ we arrive at

$$
\langle x, \nabla w(x)\rangle=\frac{\partial w}{\partial x_{1}}(1,0) w(x) \quad \text { whenever } \quad x \in D(\tau) .
$$

Thus in polar coordinates,

$$
r w_{r}(r, \theta)=w_{r}(1,0) w(r, \theta) .
$$

Dividing this equality by $r w(r, \theta)$ and integrating with respect to $r$, we find after exponentiating that (11.54) holds with $\lambda=w_{r}(1,0)$. To avoid confusion we now write $\lambda(\tau)$ for $\lambda$ in (11.54). Next we show that

$\lambda$ is non-increasing on $[\pi / 2, \pi)$ with $\lambda(\pi / 2)=1$ and $\lambda(\pi)=\lim _{\tau \rightarrow \pi} \lambda(\tau) \geq 1-1 / p$.

To prove (11.55) let $K(\tau)$ be the compact convex set $\left(\mathbb{R}^{2} \backslash D(\tau)\right) \cap\left\{\left(x_{1}, x_{2}\right) \in \mathbb{R}^{2}\right.$ : $\left.x_{1} \geq-1\right\}$ and let $\zeta=\zeta(\cdot, \tau)$ be the $\tilde{\mathcal{A}}=\nabla \tilde{f}$-harmonic Green's function for $\mathbb{R}^{2} \backslash K(\tau)$ with pole at $\infty$.

Let $z$ be a point on $\partial K(\tau)$ with polar coordinates $r=1 / 2, \theta=\tau$. Define $w^{+}$and $\zeta^{+}$in $B(z, 1 / 4)$ by $w^{+}=w$ and $\zeta^{+}=\zeta$ at points $x$ in $D(\tau) \cap B(z, 1 / 4)$ with $x_{2}>0$ while $w^{+}=\zeta^{+} \equiv 0$ in the rest of $B(z, 1 / 4)$. Using Lemma 8.3 in $D(\tau) \cap B(z, 1 / 4)$ to compare $w^{+} / \zeta^{+}$and then Harnack's inequality we see that $w(1 / 2, \theta) \approx \zeta(1 / 2, \theta)$ for $0 \leq \theta<\tau$ where ratio constants depend only on the data so are independent of $\tau \in[\pi / 2, \pi)$. Applying the same argument below the $x_{2}$ axis we get the above ratio for $r=1 / 2$ and $0 \leq|\theta|<\tau$. So by the maximum principle for $\tilde{\mathcal{A}}$-harmonic functions we have

$$
w(x) \approx \zeta(x) \text { for } x \in D(\tau) \cap \bar{B}(0,1 / 2)
$$

where ratio constants depend only on the data. From (11.56) and the maximum principle for $\tilde{\mathcal{A}}$-harmonic functions, we have with $x_{1}=r, x_{2}=0,0 \leq r \leq 1 / 2$, and $\pi / 2 \leq \tau_{1}<\tau_{2}<\pi$

$$
w\left(r, 0, \tau_{1}\right) \approx \zeta\left(r, 0, \tau_{1}\right)<\zeta\left(r, 0, \tau_{2}\right) \approx w\left(r, 0, \tau_{2}\right) .
$$

Letting $r \rightarrow 0$ it follows that necessarily $\lambda\left(\tau_{2}\right) \leq \lambda\left(\tau_{1}\right)$. Also $w(\cdot, \pi / 2)=x_{1}$ is $\tilde{\mathcal{A}}-$ harmonic in $\left\{\left(x_{1}, x_{2}\right) \in \mathbb{R}^{2} ; x_{1}>0\right\}$ with continuous zero boundary value on $\left\{x_{1}=0\right\}$ and has value 1 at $(1,0)$. From the uniqueness of $w$, we conclude that $\lambda(\pi / 2)=1$ To prove the right hand inequality in (11.55) we first observe from comparison of $w^{+}$to a linear function that vanishes at points with polar coordinate $\theta=\tau$ and also to one that vanishes at points with coordinate $\theta=-\tau$, as well as the argument used in the derivation of (11.41) that

$$
c^{-1} \leq|\nabla w(1 / 2, \pm \tau)| \leq c
$$


where $c$ depends only on the structure assumptions for $f$ and $p$ so is independent of $\tau$. Using this fact, $(8.9)(a)$, and the homogeneity of $w$ we see that

$$
\infty>\int_{\partial D(\tau) \cap B(0,1 / 2)} \tilde{f}(\nabla w(\cdot, \tau)) d \mathcal{H}^{1} \approx \int_{0}^{1} r^{(\lambda(\tau)-1) p} d r
$$

where ratio constants are independent of $\tau \in(3 \pi / 4, \pi)$. Clearly, (11.59) implies the exponent in the integral is larger than -1 so $\lambda(\tau)>1-1 / p$. Letting $\tau \rightarrow \pi$ we get the right hand inequality in (11.55). Next using (3.1)-(3.4), (11.54), and Ascoli's theorem we see that a subsequence of $\{w(\cdot, \pi-1 / m)\}$ converges to $v$ which is continuous on $\mathbb{R}^{2}$ with $v \equiv 0$ on $\left\{\left(x_{1}, 0\right) \in \mathbb{R}^{2}: x_{1} \leq 0\right\}, v(1,0)=1$, and $\tilde{\mathcal{A}}$-harmonic in $\mathbb{R}^{2} \backslash\left\{\left(x_{1}, 0\right) \in \mathbb{R}^{2}: x_{1} \leq 0\right\}$ satisfying

$$
v(t x)=t^{\lambda(\pi)} v(x) \quad \text { where } \lambda(\pi) \geq 1-1 / p .
$$

To show $\lambda(\pi)=1-1 / p$ we let $0<\delta<10^{-10}$ be a small but fixed positive number. Also $\epsilon>0,0<\epsilon<<\delta^{100}$ is allowed to vary. We put $\tau=\pi-\epsilon$ and write $\zeta$ and $w$ for $\zeta(\cdot, \tau)$ and $w(\cdot, \tau)$. If $p=2$ we note from (10.36) and existence for discrete measures in Theorem B as well as (11.3) that

$$
\begin{aligned}
\gamma & =2 \int_{\partial K(\tau)}\langle y, \nabla \zeta(y)\rangle \tilde{f}(\nabla \zeta(y))|\nabla \zeta(y)|^{-1} d \mathcal{H}^{1} \\
& =2 \int_{\partial K(\tau)}\langle y+(1,0), \nabla \zeta(y)\rangle \tilde{f}(\nabla \zeta(y))|\nabla \zeta(y)|^{-1} d \mathcal{H}^{1} .
\end{aligned}
$$

While if $p>2$ we get from the same reasoning and (10.37) that

$$
\begin{aligned}
1 & \approx \frac{p-2}{p-1} \mathcal{C}_{\tilde{\mathcal{A}}}(K(\tau))^{1 /(p-1)} \\
& =p \int_{\partial K(\tau)}\langle y+(1,0), \nabla \zeta(y)\rangle \tilde{f}(\nabla \zeta(y))|\nabla \zeta(y)|^{-1} d \mathcal{H}^{1}
\end{aligned}
$$

Let $J(\tau)=K(\tau) \cap\left\{y: y_{1} \geq-1+\delta\right\}$ and let $q(\cdot)$ be the $\tilde{\mathcal{A}}$-harmonic Green's function for the complement of $I(\tau)=K(\tau) \cap\left\{x: x_{1} \leq-1+3 \delta\right\}$ with a pole at infinity. We note that $q(\cdot) \geq \zeta(\cdot)$ in $\mathbb{R}^{2}$. If $p>2$ then from the Hopf boundary maximum principle we deduce

$$
\begin{aligned}
\int_{\partial K(\tau) \backslash J(\tau)} & \langle y+(1,0), \nabla \zeta(y)\rangle \tilde{f}(\nabla \zeta(y))|\nabla \zeta(y)|^{-1} d \mathcal{H}^{1} \\
& \leq \int_{\partial I(\tau)}\langle y+(1,0), \nabla q(y)\rangle \tilde{f}(\nabla q(y))|\nabla q(y)|^{-1} d \mathcal{H}^{1} \\
& \leq c \delta^{\frac{p-2}{p-1}}
\end{aligned}
$$

where $c$ depends only on the data. Here we once again used the existence for discrete measures in Theorem B, (11.3), and Remark 11.3. If $p=2$ let $z_{0}$ be the point with polar coordinate $r=1+\delta$ and $\theta=\pi$. Applying the same argument as in the derivation 
of (11.56) we obtain

$$
\zeta(x) / q(x) \approx \zeta\left(z_{0}\right) / q\left(z_{0}\right) \text { when } x \in D(\tau) \text { and }|x+(1,0)|<2 \delta .
$$

From $(3.5)(i i)$ we observe that $q\left(z_{0}\right) \approx 1$. Also since $\partial K(\tau)$ is uniformly $(1,2)$-fat we conclude from (3.1) (iii) that there exists $\tilde{\sigma}$ with $\zeta\left(z_{0}\right) \leq \delta^{\tilde{\sigma}}$. Using these inequalities, (11.64), (11.61) with $\zeta$ replaced by $q$, and the Hopf boundary maximum principle (as in (11.63)) it follows that if $p=2$,

$$
\int_{\partial K(\tau) \backslash J(\tau)}\langle y+(1,0), \nabla \zeta(y)\rangle \tilde{f}(\nabla \zeta(y))|\nabla \zeta(y)|^{-1} d \mathcal{H}^{1} \leq c \delta^{\tilde{\sigma}}
$$

where $c$ and $\tilde{\sigma}$ depend only on the data. To finish the proof of Lemma 11.6 we use (11.63), (11.65), (11.61), (11.62), and the fact that $\langle x+(1,0), \nabla \zeta\rangle=\sin (\epsilon)|\nabla \zeta|$ on $\partial J(\tau)$ to first get for $\delta$ sufficiently small that

$$
c^{-1} \leq \epsilon \int_{\partial J(\tau) \cap \partial K(\tau)} \tilde{f}(\nabla \zeta(y)) d \mathcal{H}^{1} .
$$

Second as in (11.56) we see that $\zeta \leq c(\delta) w$ in $D(\tau) \cap B(0,1-\delta / 2)$. Using this inequality and the Hopf boundary maximum principle a final time we find in view of (11.58) and (11.66) that

$$
\begin{aligned}
c^{-1} & \leq c(\delta) \epsilon \int_{\partial J(\tau) \cap \partial K(\tau)} \tilde{f}(\nabla w(y)) d \mathcal{H}^{1} \\
& \leq c c(\delta) \epsilon \int_{0}^{1} r^{(\lambda(\tau)-1) p} d r \\
& =\frac{c c(\delta) \epsilon}{(\lambda(\tau)-1) p+1}
\end{aligned}
$$

Letting $\epsilon \rightarrow 0$ we conclude that $(\lambda(\tau)-1) p+1 \rightarrow 0$ as $\tau \rightarrow \pi$ so $\lambda(\pi)=1-1 / p$.

Armed with Lemma 11.6 we now can prove (11.52) and so finish the proof of Proposition 11.5. We use the same notation as in Proposition 11.4 except we assume $E \subset P=\left\{x: x_{2}=0\right\}$. Let $\left\{Q_{k}\right\}$ be a Whitney decomposition of the interior of $E$ considered as a subset of $P$. Let $Q \in\left\{Q_{k}\right\}$, and let $z=\left(z_{1}, 0, z_{3}, \ldots, z_{n}\right)$ be a point in $\partial^{\prime} E$ with $d^{\prime}(z, Q) \approx s(Q)$. From convexity of $E$ we see that there is a $(n-2)$ dimensional plane, say $P_{1}$ containing $z$ with the property that $E$ is contained in the closure of one of the components of $P \backslash P_{1}$. Rotating $P_{1}$ if necessary we may assume that

$P_{1}=\left\{x \in \mathbb{R}^{n}: x_{1}=z_{1}, x_{2}=0\right\} \quad$ and $E \subset \Omega=\left\{x \in \mathbb{R}^{n}: x_{1}-z_{1} \leq 0\right.$ and $\left.x_{2}=0\right\}$.

Let $v$ be as in Lemma 11.6. We extend $v$ continuously to $\mathbb{R}^{n}$ (also denoted $v$ ) by defining this function to be constant in the other $(n-2)$ coordinate directions. Then $\hat{v}(x)=v(x-z)$ for $x \in \mathbb{R}^{n}$ is $\mathcal{A}$-harmonic in $\mathbb{R}^{n} \backslash \Omega$. Comparing boundary values and using the maximum principle, as well as Lemmas 5.2 and 5.3 we deduce that

$$
C U(x) \geq \hat{v}(x) \quad \text { whenever } x \in B(0,2 \rho),
$$


where $C$ depends only on the data and $\mathcal{C}_{\mathcal{A}}(E)$. As in Proposition 11.4 we see that

$$
\left.c^{\prime} \int_{Q} f\left(\nabla U_{+}\right) d \mathcal{H}^{n-1} \geq U\left(y_{Q}\right)\right)^{p} s(Q)^{n-1-p} .
$$

Now from Lemma 11.6 we also deduce that

$$
c^{\prime \prime} \hat{v}\left(y_{Q}\right) \geq s(Q)^{1-1 / p}
$$

Combining (11.68)-(11.70) we conclude that for some $\tilde{c}$ with the same dependence as the above constants,

$$
\tilde{c} \int_{Q} f\left(\nabla U_{+}\right) d \mathcal{H}^{n-1} \geq s(Q)^{n-2} .
$$

Now since $B(0,4 a) \cap P \subset E$ we see for $l$ large from Lipschitz starlikeness of $E$ that there are at least $\approx 2^{l(n-2)}$ members of $\left\{Q_{k}\right\}$ whose side length lies between $2^{-l-1} a$ and $2^{-l} a$. Using this fact and summing (11.71) we get Proposition 11.5.

11.3. Uniqueness in Theorem B. Uniqueness in Theorem B follows from Theorem $\mathrm{A}$ as in [CNSXYZ] (see also [AGHLV]). To give the reader the gist of the argument we consider the simplest $p=n$ case. Suppose $\mu$ is a positive finite Borel measure on $\mathbb{S}^{n-1}$ satisfying (7.1) and let $E_{0}$ and $E_{1}$ be two compact convex sets with nonempty interiors for which the corresponding $\mathcal{A}$-harmonic Green's functions satisfy(7.2) $(a)-(c)$ in Theorem B relative to $\mu$. Let $h_{0}$ and $h_{1}$ be the support functions of $E_{0}$ and $E_{1}$ respectively. For $t \in[0,1]$ we let $E_{t}=(1-t) E_{0}+t E_{1}$ and put $\mathbf{m}(t)=\mathcal{C}_{\mathcal{A}}\left(E_{t}\right)$ for $t \in[0,1]$. Using (11.30) and (10.36), we deduce that

$$
\left.\frac{d}{d t} \mathbf{m}(t)\right|_{t=0}=n \gamma^{-1} \mathcal{C}_{\mathcal{A}}\left(E_{0}\right) \int_{\mathbb{S}^{n-1}}\left(h_{1}(\xi)-h_{0}(\xi)\right) d \mu(\xi)=0 .
$$

From Theorem A we see that $m$ is concave on $[0,1]$. Using this fact (11.72) we find that

$$
\mathbf{m}^{\prime}(0)=0 \geq \mathbf{m}(1)-\mathbf{m}(0) .
$$

with equality only if $m$ is constant on $[0,1]$. From $(11.73)$ we first get $\mathcal{C}_{\mathcal{A}}\left(E_{0}\right)=$ $\mathbf{m}(0) \geq \mathbf{m}(1)=\mathcal{C}_{\mathcal{A}}\left(E_{1}\right)$ and second by reversing the roles of $E_{0}$ and $E_{1}$, we get $\mathcal{C}_{\mathcal{A}}\left(E_{1}\right)=\mathbf{m}(1)=\mathbf{m}(0)=\mathcal{C}_{\mathcal{A}}\left(E_{0}\right)$. We conclude that $\mathbf{m}$ is constant and therefore equality holds in (2.4) of Theorem $\mathrm{A}$ and so $E_{0}$ is a translation and dilation of $E_{1}$. Using Remark 5.5 and the fact that $\mathcal{C}_{\mathcal{A}}\left(E_{0}\right)=\mathcal{C}_{\mathcal{A}}\left(E_{1}\right)$ we see that honest dilations are not possible when $p=n$. For $p>n$, by considering $\mathbf{m}(t)=C_{\mathcal{A}}\left(E_{t}\right)^{1 /(p-n)}$, a similar argument can be used to prove uniqueness of $E$ up to translation. This finishes our outline of the proof of uniqueness in Theorem $\mathrm{B}$ for $n \geq p$.

\section{ACKNOWLEDGMENT}

This material is based upon work supported by National Science Foundation under Grant No. DMS-1440140 while the first and the third authors were in residence at the MSRI in Berkeley, California, during the Spring 2017 semester. The second author 
was partially supported by NSF DMS-1265996. The third author was partially supported by the Hausdorff Center for Mathematics as well as DFG-SFB 1060. Research for this article was carried out while the first and fourth author were visiting the department of mathematics at the University of Kentucky, the authors would like to thank the department for its hospitality.

\section{REFERENCES}

[A] Murat Akman. On the dimension of a certain measure in the plane. Ann. Acad. Sci. Fenn. Math., 39(1):187-209, 2014.

[AGHLV] M. Akman, J. Gong, J. Hineman, J. Lewis, and A. Vogel. The Brunn-Minkowski inequality and a Minkowski problem for nonlinear capacity. arXiv:1709.00447, Submitted(2017).

$[\mathrm{AH}] \quad$ David R. Adams and Lars Inge Hedberg. Function spaces and potential theory, volume 314 of Grundlehren der Mathematischen Wissenschaften [Fundamental Principles of Mathematical Sciences]. Springer-Verlag, Berlin, 1996.

[ALV] Murat Akman, John Lewis, and Andrew Vogel. $\sigma$-finiteness of elliptic measures for quasilinear elliptic PDE in space. Adv. Math., 309:512-557, 2017.

[ALV12] Murat Akman, John L. Lewis, and Andrew Vogel. On the logarithm of the minimizing integrand for certain variational problems in two dimensions. Anal. Math. Phys., 2(1):7988, 2012.

[B1] Christer Borell. Capacitary inequalities of the Brunn-Minkowski type. Math. Ann., 263(2):179-184, 1983.

[B2] Christer Borell. Hitting probabilities of killed Brownian motion: a study on geometric regularity. Ann. Sci. École Norm. Sup. (4), 17(3):451-467, 1984.

[CC] Andrea Colesanti and Paola Cuoghi. The Brunn-Minkowski inequality for the $n$ dimensional logarithmic capacity of convex bodies. Potential Anal., 22(3):289-304, 2005.

[CJL] Luis A. Caffarelli, David Jerison, and Elliott H. Lieb. On the case of equality in the Brunn-Minkowski inequality for capacity. Adv. Math., 117(2):193-207, 1996.

[CNSXYZ] A. Colesanti, K. Nyström, P. Salani, J. Xiao, D. Yang, and G. Zhang. The Hadamard variational formula and the Minkowski problem for p-capacity. Adv. Math., 285:15111588, 2015.

[CS] Andrea Colesanti and Paolo Salani. The Brunn-Minkowski inequality for $p$-capacity of convex bodies. Math. Ann., 327(3):459-479, 2003.

[CS1] Andrea Cianchi and Paolo Salani. Overdetermined anisotropic elliptic problems. Math. Ann., 345(4):859-881, 2009.

[EL] Alexandre Eremenko and John L. Lewis. Uniform limits of certain $A$-harmonic functions with applications to quasiregular mappings. Ann. Acad. Sci. Fenn. Ser. A I Math., 16(2):361-375, 1991.

[G] R. J. Gardner. The Brunn-Minkowski inequality. Bull. Amer. Math. Soc. (N.S.), 39(3):355-405, 2002.

[GL] Nicola Garofalo and Fang-Hua Lin. Unique continuation for elliptic operators: a geometric-variational approach. Comm. Pure Appl. Math., 40(3):347-366, 1987.

[GM] J. B. Garnett and D. E. Marshall. Harmonic measure, volume 2 of New Mathematical Monographs. Cambridge University Press, Cambridge, 2008. Reprint of the 2005 original.

[GT] David Gilbarg and Neil S. Trudinger. Elliptic partial differential equations of second order. Classics in Mathematics. Springer-Verlag, Berlin, 2001. Reprint of the 1998 edition.

[Ga] R. M. Gabriel. An extended principle of the maximum for harmonic functions in 3dimensions. J. London Math. Soc., 30:388-401, 1955.

[HKM] Juha Heinonen, Tero Kilpeläinen, and Olli Martio. Nonlinear Potential Theory of Degenerate Elliptic Equations. Dover Publications Inc., 2006. 
[J] David Jerison. A Minkowski problem for electrostatic capacity. Acta Math., 176(1):1-47, 1996.

[KZ] Tero Kilpeläinen and Xiao Zhong. Growth of entire $\mathcal{A}$-subharmonic functions. Ann. Acad. Sci. Fenn. Math., 28(1):181-192, 2003.

[Kr] I. N. Krol. The behavior of the solutions of a certain quasilinear equation near zero cusps of the boundary. Trudy Mat. Inst. Steklov., 125:140-146, 233, 1973. Boundary value problems of mathematical physics, 8 .

[LLN] John Lewis, Niklas Lundström, and Kaj Nyström. Boundary Harnack inequalities for operators of $p$-Laplace type in Reifenberg flat domains. In Perspectives in partial differential equations, harmonic analysis and applications, volume 79 of Proc. Sympos. Pure Math., pages 229-266. Amer. Math. Soc., Providence, RI, 2008.

[LN] John Lewis and Kaj Nyström. Boundary behaviour for $p$ harmonic functions in Lipschitz and starlike Lipschitz ring domains. Ann. Sci. École Norm. Sup. (4), 40(5):765-813, 2007.

[LN1] John Lewis and Kaj Nyström. Boundary behavior and the Martin boundary problem for $p$ harmonic functions in Lipschitz domains. Ann. of Math. (2), 172(3):1907-1948, 2010.

[LN2] John Lewis and Kaj Nyström. Regularity and free boundary regularity for the $p$ Laplacian in Lipschitz and $C^{1}$ domains. Ann. Acad. Sci. Fenn. Math., 33(2):523-548, 2008.

[LN3] John Lewis and Kaj Nyström. Regularity and free boundary regularity for the $p$-Laplace operator in Reifenberg flat and Ahlfors regular domains. J. Amer. Math. Soc., 25(3):827862, 2012.

[LN4] John Lewis and Kaj Nyström. Quasi-linear PDEs and low-dimensional sets. J. Eur. Math. Soc. (JEMS), 20(7):1689-1746, 2018.

[La] N. S. Landkof. Foundations of modern potential theory. Springer-Verlag, New YorkHeidelberg, 1972. Translated from the Russian by A. P. Doohovskoy, Die Grundlehren der mathematischen Wissenschaften, Band 180.

[Li] Gary M. Lieberman. Boundary regularity for solutions of degenerate elliptic equations. Nonlinear Anal., 12(11):1203-1219, 1988.

[MSZ] Jan Malý, David Swanson, and William P. Ziemer. The co-area formula for Sobolev mappings. Trans. Amer. Math. Soc., 355(2):477-492, 2003.

[S] James Serrin. Local behavior of solutions of quasi-linear equations. Acta Mathematica, 111:247-302, 1964. 10.1007/BF02391014.

[Sc] Rolf Schneider. Convex bodies: the Brunn-Minkowski theory, volume 44 of Encyclopedia of Mathematics and its Applications. Cambridge University Press, Cambridge, 1993.

[T] Peter Tolksdorf. Regularity for a more general class of quasilinear elliptic equations. $J$. Differential Equations, 51(1):126-150, 1984. 
Murat Akman, Department of Mathematics, University of Connecticut, Storrs, CT 06269-1009

E-mail address: murat.akman@uconn.edu

Web-page: http://www.math.uconn.edu/〜akman/

John Lewis, Department of Mathematics, University of Kentucky, Lexington, KenTUCKY, 40506

E-mail address: johnl@uky.edu

Web-page: http://www.ms.uky.edu/ johnl/

Olli Saari, Mathematisches Institut, Endenicher Allee 60, 53115 Bonn, Germany

E-mail address: saari@math.uni-bonn.de

Web-page: https://www.math.uni-bonn.de/people/saari/

Andrew Vogel, Department of Mathematics, Syracuse University, Syracuse, New YORK 13244

E-mail address: alvogel@syracuse.edu 